\title{
Multiscale Simulation of Temperature- and Pressure-dependent Nonlinear Dynamics of PMMA/CNT Composite Plates
}

\section{J.F. Wang ( $\nabla$ jf.wang@bjut.edu.cn )}

Beijing University of Technology https://orcid.org/0000-0003-1575-4473

S.Q Shi

Beijing University of Technology

Y.Z. Liu

Beijing Institute of Technology

J.P. Yang

Beijing Institute of Technology

Lik-ho Tam

Beihang University

\section{Research Article}

Keywords: Composite structure, Nonlinear dynamics, Aerodynamic, Multiscale simulation, Efficiency parameter

Posted Date: November 30th, 2021

DOI: https://doi.org/10.21203/rs.3.rs-1079170/v1

License: (c) (i) This work is licensed under a Creative Commons Attribution 4.0 International License. Read Full License 


\title{
Multiscale simulation of temperature- and pressure- dependent nonlinear dynamics of PMMA/CNT composite plates
}

\author{
J.F. Wang ${ }^{\mathrm{a}, *}$, S.Q. Shi ${ }^{\mathrm{a}}$, Y.Z. Liu ${ }^{\mathrm{a}}$, J.P. Yang ${ }^{\mathrm{a}}$, Lik-ho Tam, ${ }^{\text {b* }}$ \\ ${ }^{a}$ Beijing Key Laboratory of Nonlinear Vibrations and Strength of Mechanical \\ Structures, Faculty of Materials and Manufacturing, Beijing University of \\ Technology, Beijing 100124, China \\ ${ }^{\mathrm{b}}$ School of Transportation Science and Engineering, Beihang University, Beijing \\ 100191, China
}

* Corresponding author, email: jf.wang@bjut.edu.cn (J.F. Wang); leo_tam@buaa.edu.cn (Lik-ho Tam) 


\begin{abstract}
Owing to the excellent mechanical properties, polymethyl methacrylate (PMMA)/carbon nanotube (CNT) composite has been increasingly adopted in the aerospace field, which is usually subjected to various temperature conditions and supersonic aerodynamic loads. Using a molecular dynamics (MD)-based multiscale simulation, the nonlinear forced vibration of PMMA/CNT composite plate is investigated under coupled temperature and aerodynamic loads conditions. The longitudinal, transverse, and shear moduli of PMMA/single-walled CNT (SWCNT) nanocomposite obtained from MD simulation at different temperature and pressure levels are substituted into the extended rule of mixtures to establish the constitutive equations of PMMA/CNT composite plate. Meanwhile, Poisson's ratio and thermal expansion coefficient of nanocomposite are used in the constitutive equations. Based on the third-order shear deformation theory, von-Karman nonlinear strain-displacement relation, and Hamilton's principle, the partial differential equation of composite plate is derived, which is reduced into a set of coupled ordinary differential equations by applying Galerkin method and is solved using the fourth-order Runge-Kutta method. The phase portraits and time histories of composite plate are obtained under the complex loads including transverse harmonic excitation and aerodynamic pressure, which are applied to analyze the dynamic characteristics of system. This study reveals the nonlinear dynamic characteristics of PMMA/CNT composite plate, which contributes to the prediction of long-term performance of composite materials in aerospace field.
\end{abstract}

Keywords: Composite structure; Nonlinear dynamics; Aerodynamic; Multiscale simulation; Efficiency parameter 


\section{Introduction}

Polymethyl methacrylate (PMMA) has been widely applied as a replacement for glass in the applications of porthole and windshield of airplane owing to the excellent transparency and easy processing $[1,2]$. However, as the PMMA is fragile in stress concentration zone and possesses low melting temperature, it is considered as one of the vulnerable parts of the airplane. Because of the superior mechanical properties and the high resistance to thermal stress, PMMA/carbon nanotube (CNT) composite has been increasingly adopted in the aerospace application $[3,4]$. During the working condition, the airplane is inevitably subjected to various temperature and aerodynamic pressure, which cause a series of complex nonlinear dynamic behaviors [5-10]. Specifically, the increase of temperature level leads to the reduced fundamental frequencies and the increased nonlinear to linear frequency ratios of composite plate, which affect the structural dynamic response of the airplane. Meanwhile, the sequence of modes of the composite plate changes with the increased aerodynamic pressure [11]. The long-term vibration phenomenon at different temperature and aerodynamic pressure levels usually leads to the unpredictable dynamic behavior and the consequent failure of composite structure. In order to understand the long-term performance of PMMA/CNT composite in the airplane, it is a key issue to investigate the nonlinear dynamic response of PMMA/CNT composite in the complex temperature and aerodynamic pressure conditions.

Previous experimental works have focused on the vibration response of composite plate. With the help of microcontroller-type thermal chamber and the impact-type vibration analyzer with virtual programming, the experimental frequency of CNTreinforced composite $(\mathrm{CNTRC})$ plate was recorded under the elevated temperature conditions [12]. It was shown that the vibration frequency of CNTRC plate decreased with the increase of temperature, as the stiffness of plate decreased [12]. Meanwhile, the oscillation amplitudes of cantilevered flexible plate under the effect of axial flow were measured in a low-turbulence wind tunnel with the increased flow velocity by a 
laser sensor [13]. It was shown that when the flow velocity was relatively low but still above the flutter point, the oscillations of plates were periodic and almost harmonic according to the measured time history of displacement. However, due to complex environment and aerodynamic pressure conditions, it is difficult to setup the testing equipment and condition in the experiment, and to interpret the mechanism of nonlinear vibration inside the composite structure via vibration experiment.

The numerical simulation has been considered as an effective approach in analyzing the nonlinear behaviors of composite in the complex environment and load conditions [14-17]. It was found that with the temperature increased from 300 to 700 $\mathrm{K}$, the natural frequency of PMMA/CNT composite plate decreased and the ratio of nonlinear to linear frequencies increased, which indicated that the increased temperature enhanced the nonlinear behavior of the plate $[10,18,19]$. Meanwhile, it was reported that the maximum deflection of functionally graded (FG)-based PMMA/CNT composite plate occurred at the center of the flat panel without the aerodynamic pressure, while for the flat panel subjected to the aerodynamic pressures, the maximum deflection of the panel moved to the opposite direction of the airflow and the vibration mode shapes were changed accordingly [20, 21]. From these previous investigations, it is found that both temperature and aerodynamic pressure conditions cause the complex nonlinear behavior of composite system. However, it is not clear about the nonlinear dynamic response of PMMA/CNT composite plate in the coupled effect of temperature and pressure conditions. Meanwhile, the mechanism of nonlinear dynamic response under the complex environment and aerodynamic pressure conditions is still lacking for composite structures.

In order to fully understand the complex dynamic response of CNT-reinforced polymer composite, the MD-based multiscale approach was developed to investigate the nonlinear vibration of PMMA/CNT composite plate [22-25]. In our recent simulation study, it was reported that the distributed CNT close to top and bottom surfaces caused high natural frequency and low nonlinear frequency of PMMA/CNT composite plate, as the CNT distribution is efficient to increase the stiffness of 
composite plate [26]. Meanwhile, the effect of CNT volume fraction was studied, where the natural frequency of FG-based PMMA/CNT composite plate increased with the increased CNT volume fraction, and the ratio of nonlinear to linear frequency decreased correspondingly due to the enhanced stiffness [27]. Furthermore, the nonlinear dynamic responses of FG-based PMMA/CNT composite beam were investigated under the axial airflow in thermal conditions [28]. It was demonstrated that the thermal stress induced by the temperature difference significantly reduced the the flutter boundary. In addition, it was shown that with the elevated aerodynamic pressure, the first-order frequency of FG-CNTRC panel increased, while the second-order frequency decreased gradually, and the frequency coalescence between the first and second modes leads to the flutter instability [29]. Among these studies, the temperature-independent efficiency parameters are introduced into the extended rule of mixtures (EROM), and the prediction of CNT-reinforced composite plate may not be strictly accurate as the effect of temperature is not considered in the calculated mechanical properties. Meanwhile, there are few investigations relevant to the nonlinear dynamic responses in the coupled temperature and aerodynamic conditions.

In this paper, the nonlinear dynamic response of PMMA/CNT composite plate is investigated in the complex temperature and aerodynamic pressure conditions. The longitudinal, transverse, shear moduli, Poisson's ratio, and thermal expansion coefficient (TEC) of PMMA/SWCNT nanocomposite are determined using MD simulation at different temperature and pressure levels. Subsequently, the obtained elastic properties from MD simulation are substituted into the EROM to calculate the effective longitudinal, transverse, and shear moduli of PMMA/CNT composite plate. Both TEC and elastic properties are applied to establish the constitutive equations of composite plate in the partial differential equations, which are then reduced into a set of coupled nonlinear ordinary differential equations using Galerkin method. The ordinary differential equations are solved using the fourth-order Runge-Kutta method to obtain the time histories and phase portraits of PMMA/CNT composite. This study contributes to the nonlinear dynamic behavior of PMMA/CNT composite plate in the 
complex temperature and aerodynamic pressure conditions.

\section{Molecular simulations}

The MD simulation adopted in this study include the model construction of SWCNT, PMMA, and PMMA/SWCNT nanocomposite, and the structural relaxation and dynamic deformation. After that, the longitudinal, transverse, shear moduli, Poisson's ratio, and TEC of molecular models are obtained, which are applied as the inputs in the constitutive equation for the subsequent macromechanical simulations. The details of modeling and simulation are provided in the following sections.

\subsection{Force field and molecular model}

In the molecular simulation, the Tersoff potential is applied to characterize the interactions of SWCNT, which consists of two-body interactions, three-body interactions, and multibody term [30]. Meanwhile, the polymer consistent force field (PCFF) is adopted to characterize the interactions of PMMA polymer and between SWCNT and PMMA matrix in nanocomposite [31]. The potential functions in the PCFF include the bond stretching, angle bending, dihedral angle torsion, improper outof-plane, and cross-coupling terms of the bonded interactions, and the van der Waals $(\mathrm{vdW})$ and Coulombic terms of the non-bonded interactions with a cutoff distance of $1.25 \mathrm{~nm}[32,33]$. The potential functions of Tersoff and PCFF potentials are described in our recent study [26]. In the construction of the molecular models, a periodic $(5,5)$ SWCNT model with a diameter of $0.678 \mathrm{~nm}$ and a length of $6.0 \mathrm{~nm}$ is investigated, as shown in Fig. 1(a), which has been extensively adopted as the reinforcing filler in polymer/SWCNT nanocomposites [32, 34]. For the PMMA polymer, isotactic PMMA (i-PMMA) is investigated here, which is widely used in various engineering application due to the excellent mechanical properties $[35,36]$. The molecular model of i-PMMA polymer chain with fifty repeating units is shown in Fig. 1(b). The PMMA polymer developed in our previous study is used here, where thirty-one i-PMMA chains are packed into a periodic box with an initial density of $1.08 \mathrm{~g} \cdot \mathrm{cm}^{-3}$, which is close to the 
experimental range of $1.15 \sim 1.19 \mathrm{~g} \cdot \mathrm{cm}^{-3}[25,35-39]$. For the nanocomposite, the configuration is constructed that only a small number of SWCNT segments are embedded into the matrix [32]. Hence, the single $(5,5)$ SWCNT segment is placed in the center of a periodic simulation box, and thirty-one i-PMMA chains are then packed randomly around the tube in non-overlapping positions. The molecular model of nanocomposite is considered as the representative model at the molecular level, as shown in Fig. 1(c).

(a)

(c)

(d)

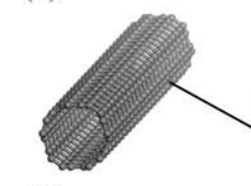

(b)

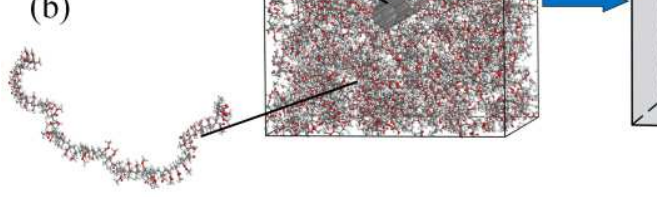

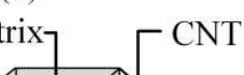

(e)

\author{
政
}


ramp rate of $100 \mathrm{~K}$ per $100 \mathrm{ps}$, and the pressure is set as $1 \mathrm{~atm}$ in each case. For PMMA polymer and PMMA/SWCNT nanocomposite, the constructed models are equilibrated in the NVE ensemble for $50 \mathrm{ps}$, and then in the NVT ensemble at $300 \mathrm{~K}$ for another 50 ps, and finally in the NPT ensemble at $300 \mathrm{~K}$ and $1 \mathrm{~atm}$ for $100 \mathrm{ps}$. After that, the PMMA polymer and PMMA/SWCNT nanocomposite models are further equilibrated using a validated equilibration procedure with seven relaxation cycles to achieve the fully relaxed state [41-44]. In every cycle, the models are firstly equilibrated in an NVT ensemble at $800 \mathrm{~K}$ temperature for $50 \mathrm{ps}$, and then at $300 \mathrm{~K}$ for another $50 \mathrm{ps}$, and finally in NPT ensemble at $300 \mathrm{~K}$ with a changing pressure. Specifically, the imposed pressure in the first three cycles increases steadily from 1 to 50,000 atm, during which the structure is compressed efficiently. In the subsequent four equilibration cycles, the imposed pressure decreases gradually to 1 atm, during which the model is decompressed efficiently. When the pressure reaches $1 \mathrm{~atm}$, the model is subjected to a final relaxation process of $800 \mathrm{ps}$ at the temperature of $300 \mathrm{~K}$ and pressure of $1 \mathrm{~atm}$.

To investigate the coupled effect of temperature and pressure conditions, the equilibrated systems are conditioned at different temperature and pressure levels. Notably, the airplane generally flies at altitude between 7 and $12 \mathrm{~km}$ above sea level. In this study, the flight condition is considered as $0.0,4.0,8.0$, and $12.0 \mathrm{~km}$, and the corresponding conditions of temperature and pressure are shown in Table 1. In order to reach these conditions, a further equilibration process is performed on the SWCNT, PMMA polymer, and nanocomposite models, with the temperature decreasing from 288.15 to $216.65 \mathrm{~K}$ and the pressure decreasing from 1 to $0.1915 \mathrm{~atm}$ respectively. In each investigated condition, the model is equilibrated in an NVT simulation for $0.5 \mathrm{~ns}$ followed by an NPT equilibration for $1.0 \mathrm{~ns}$, and the detailed process is shown in Table 1. It is noted that the cooling rate is close to those used in previous simulation studies $[26,45]$. The root-mean-squared displacement, center of mass, temperature, and potential energy of the molecular PMMA polymer and PMMA/SWCNT nanocomposite models are recorded during the $1.0 \mathrm{~ns}$ NPT equilibration in each investigated case, as shown in Fig. 2, and the constant oscillation demonstrates that the models have reached 
the equilibrium state.

Table 1 Relaxation scheme of SWCNT, PMMA polymer, and PMMA/SWCNT nanocomposite.

\begin{tabular}{cccccc}
\hline Case & Altitude $(\mathrm{km})$ & Ensemble & Temperature $(\mathrm{K})$ & Pressure (atm) & Time frame (ns) \\
\hline$\# 1$ & 0.0 & NVT & 288.15 & & 0.5 \\
& & NPT & 288.15 & 1 & 1.0 \\
$\# 2$ & 4.0 & NVT & 262.15 & $/$ & 0.5 \\
& & NPT & 262.15 & 0.6085 & 1.0 \\
$\# 3$ & 8.0 & NVT & 236.15 & $/$ & 0.5 \\
$\# 4$ & 12.0 & NPT & 236.15 & 0.3518 & 1.0 \\
& & NVT & 216.65 & $/$ & 0.5 \\
\hline
\end{tabular}
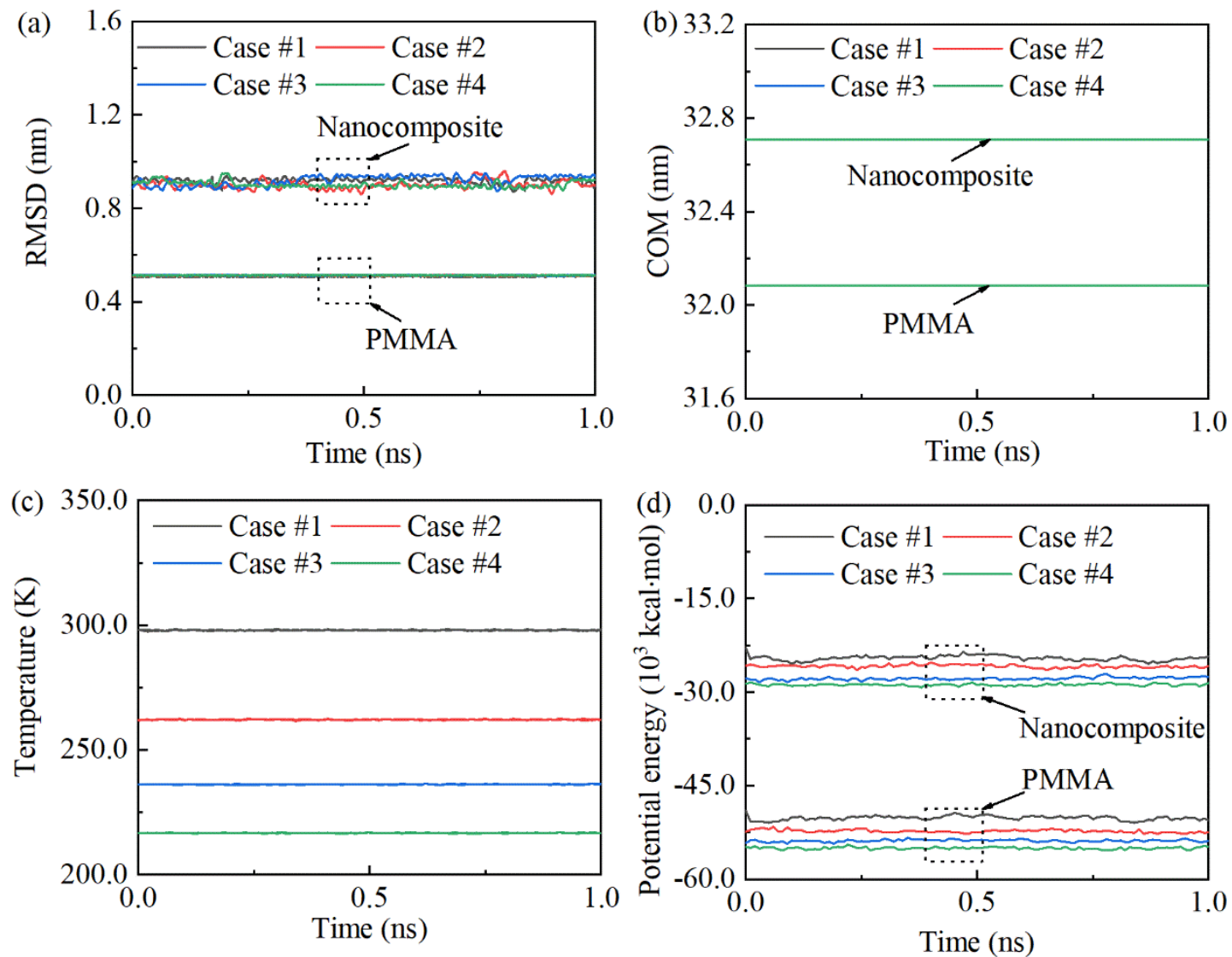

Fig. 2 Variations of (a) root-mean-squared displacement (RMSD), (b) center of mass 
(COM), (c) temperature, and (d) potential energy of PMMA polymer and PMMA/SWCNT nanocomposite during the last $1.0 \mathrm{~ns}$ NPT equilibration in each case: Case $\# 1, \# 2, \# 3$, and $\# 4$ corresponds to temperature and pressure of $0.0,4.0,8.0$, and $12.0 \mathrm{~km}$ altitude, respectively.

After the equilibration procedure, the extended equilibration is performed to measure the TEC of PMMA/SWCNT nanocomposite in each investigated condition as shown in Table 1. Specifically, the model is subjected to a stepwise cooling process starting from $288.15,262.15,236.15$, and $216.65 \mathrm{~K}$ with a ramp rate of $5 \mathrm{~K}$ per $100 \mathrm{ps}$ until the temperature drops $25 \mathrm{~K}$, respectively. In each investigated condition, the model is relaxed in the NVT ensemble for 100 ps followed by an NPT relaxation for $100 \mathrm{ps,}$ and the pressure is constant in the entire simulation. The volume and temperature are recorded in the cooling procedure. Additionally, the SWCNT is subjected to a tensile deformation in an NVT ensemble to quantify the Poisson's ratio, in which the strain rate is set as $1.6 \times 10^{10} \cdot \mathrm{s}^{-1}$, and Poisson's ratio is determined by the ratio of longitudinal strain to transverse strain. Based on the obtained models, the longitudinal, transverse, and shear moduli of SWCNT, PMMA polymer, and PMMA/SWCNT nanocomposite at different temperature and pressure levels are quantified using a constant-strain method, where the strain rates are set as $2 \times 10^{10} \mathrm{~s}^{-1}$ in the longitudinal and transverse deformations, and the shear deformation along the $x y$ plane $[46,47]$. The deformation process repeats until the strain reaches 3\%. During the entire MD simulation, the constant temperature and pressure is controlled using the Nose-Hoover thermostat and the Andersen barostat, respectively [48, 49].

\section{Macroscale simulation}

By using the extended rule of mixtures, the molecular model of PMMA/CNT nanocomposite is homogenized as a representative volume element, as shown in Fig. 1(d). Meanwhile, the properties of SWCNT, PMMA, and nanocomposite obtained from MD simulations are used as inputs to investigate the nonlinear dynamic response of 
FG-based composite plates. According to the third-order shear deformation theory, von-Karman nonlinear strain-displacement relation, and Hamilton's principle, the partial differential equation of composite plate is derived. The ordinary differential governing equation of motion is obtained by applying Galerkin method and solved by the fourth-order Runge-Kutta method. The phase portraits and time histories of FGbased composite plates at different altitudes are obtained under the transverse harmonic excitation and aerodynamic pressure to analyze the dynamic characteristics of system.

\subsection{Effective material properties}

The mechanical properties obtained from MD simulation are transformed to the input parameters of nonlinear dynamic equation of PMMA/CNT composite plate at different altitudes, as shown in Fig. 1(e). In this study, the FG-based composite plate is constructed with the thickness of $0.009 \mathrm{~m}$, the length of $0.8 \mathrm{~m}$, and the width of $0.6 \mathrm{~m}$, which is subjected to complex loads including transverse harmonic excitation $q=F \cos \Omega t$ and aerodynamic pressure, as shown in Fig. 3(a). Based on the obtained longitudinal, transverse, and shear moduli of molecular models, the efficiency parameters of PMMA/SWCNT nanocomposite are derived by applying the EROM [50]

$$
\begin{gathered}
E_{\mathrm{x}}^{\text {composite }}=\eta_{\mathrm{x}} V^{\mathrm{CNT}} E_{\mathrm{x}}^{\mathrm{CNT}}+V^{\mathrm{PMMA}} E^{\mathrm{PMMA}} \\
\frac{\eta_{\mathrm{y}}}{E_{\mathrm{y}}^{\text {composite }}}=\frac{V^{\mathrm{CNT}}}{E_{\mathrm{y}}^{\mathrm{CNT}}}+\frac{V^{\mathrm{PMMA}}}{E_{\mathrm{y}}^{\mathrm{PMMA}}} \\
\frac{\eta_{\mathrm{xy}}}{G_{\mathrm{xy}}^{\text {composite }}}=\frac{V^{\mathrm{CNT}}}{G_{\mathrm{xy}}^{\mathrm{CNT}}}+\frac{V^{\mathrm{PMMA}}}{G_{\mathrm{xy}}^{\mathrm{PMA}}}
\end{gathered}
$$

where $\eta_{\mathrm{x}}, \eta_{\mathrm{y}}$, and $\eta_{\mathrm{xy}}$ are the corresponding efficiency parameters with respect to the longitudinal, transverse, and shear deformation, respectively, $E_{\mathrm{x}}, E_{\mathrm{y}}$, and $G_{\mathrm{xy}}$ are the longitudinal, transverse, and shear modulus of SWCNT, PMMA polymer, and PMMA/SWCNT nanocomposite, respectively, $V^{\mathrm{CNT}}$ and $V^{\mathrm{PMMA}}$ are the volume fraction of SWCNT and PMMA matrix, respectively. In this study, $V^{\mathrm{CNT}}$ is set as $1.0 \mathrm{vol} \%$. The CNT distribution of the composite plate is considered with uniformly distribution (UD) and FG-X distribution, where $\mathrm{X}$ denotes the shape of CNT distribution, as shown in Fig. 3(b-c). 
(a)

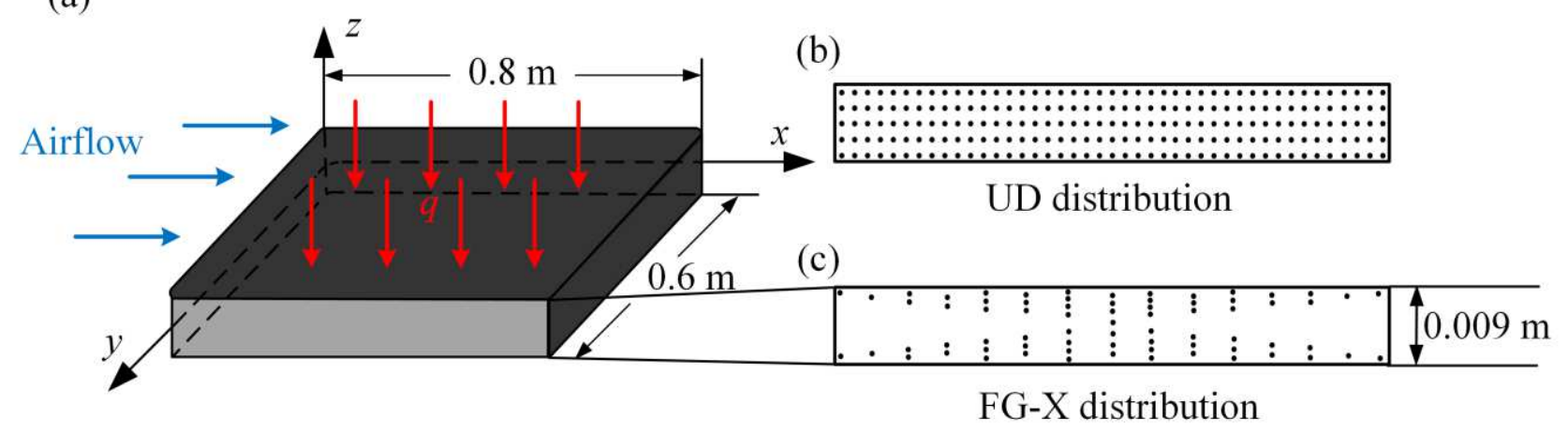

Fig. 3 The schematic diagram of (a) FG-based composite plate with the CNT distribution of: (b) uniform distribution (UD), and (c) functionally graded distribution (FG-X), where X denotes the shape of CNT distribution.

The mathematical expressions of the two distributions are given as follows

$$
\begin{gathered}
V^{\mathrm{CNT}}(z)=V_{*}^{\mathrm{CNT}} \quad \mathrm{UD} \\
V^{\mathrm{CNT}}(z)=\frac{4|z|}{h} V_{*}^{\mathrm{CNT}} \quad \mathrm{FG}-\mathrm{X}
\end{gathered}
$$

where $V_{*}^{\mathrm{CNT}}$ is the CNT volume fraction of composite plate, $h$ denotes the thickness of the plate, and $z$ is the coordinate along the thickness direction. By substituting $V^{\mathrm{CNT}}$ into Eq. (1), the longitudinal, transverse, and shear moduli of composite plate are obtained based on the derived efficiency parameters. Poisson's ratio of PMMA/CNT composite is derived based on the following equations

$$
\begin{gathered}
v_{\mathrm{xy}}^{\text {composite }}=V^{\mathrm{CNT}} v^{\mathrm{CNT}}+V^{\mathrm{PMMA}} v^{\mathrm{PMMA}} \\
v^{\mathrm{PMMA}}=\frac{E^{\mathrm{PMMA}}}{2 G_{\mathrm{xy}}^{\mathrm{PMMA}}}-1
\end{gathered}
$$

where $v_{\mathrm{xy}}^{\text {composite }}, v^{\mathrm{CNT}}$, and $v^{\mathrm{PMMA}}$ are Poisson's ratio of PMMA/SWCNT nanocomposite, SWCNT, and PMMA polymer, respectively. Meanwhile, the linear TEC of composite is derived using the following equation [22]

$$
\alpha=\frac{L_{1}-L_{0}}{L_{0} \Delta T}
$$


where $\alpha$ is the linear TEC of composite, $L_{0}$ is the initial length, $L_{\mathrm{i}}$ is the final length, and $\Delta T$ is the temperature rise. For the plate subjected to the high supersonic flow, the quasi-steady first-order piston theory is commonly used [51-53]

$$
P_{\mathrm{a}}=-\frac{\rho_{\mathrm{a}} V_{\mathrm{a}}^{2}}{\sqrt{M_{\mathrm{a}}^{2}-1}}\left[\frac{\partial w}{\partial x}+\left(\frac{M_{\mathrm{a}}^{2}-2}{M_{\mathrm{a}}^{2}-1}\right) \frac{1}{V_{\mathrm{a}}} \frac{\partial w}{\partial t}\right]
$$

where $P_{\mathrm{a}}, M_{\mathrm{a}}$ and $t$ are the aerodynamic pressure, Mach number and time, respectively, $w$ is the transverse displacement of the plate in the middle plane. $V_{\mathrm{a}}$ and $\rho_{\mathrm{a}}$ denote the velocity of incoming flow and air mass density, respectively, which are obtained via the ideal gas law [54] and Bernoulli equation [55]

$$
\begin{gathered}
\rho_{\mathrm{a}}=\frac{P_{\mathrm{i}} M}{T_{\mathrm{i}} R^{*}} \\
P_{\mathrm{i}}+0.5 \rho_{\mathrm{a}} V_{\mathrm{a}}^{2}+\rho_{\mathrm{a}} g h=P_{0}
\end{gathered}
$$

where $P_{\mathrm{i}}$ and $T_{\mathrm{i}}$ are the pressure and temperature in Case \#i $(\mathrm{i}=1,2,3,4)$, and $M, R^{*}, g, h$, and $P_{0}$ are the molar mass of air, universal gas constant, earth-surface gravitational acceleration, altitude above sea level, and sea level standard atmospheric pressure of 1 atm, respectively.

\subsection{Governing equations}

Reddy's third-order shear deformation theory [56] is adopted to establish the governing equations as follows

$$
\begin{gathered}
u(x, y, z, t)=u_{0}(x, y, t)+z \phi_{\mathrm{x}}(x, y, t)-c_{1} z^{3}\left(\phi_{\mathrm{x}}+\frac{\partial w_{0}}{\partial x}\right) \\
v(x, y, z, t)=v_{0}(x, y, t)+z \phi_{\mathrm{y}}(x, y, t)-c_{1} z^{3}\left(\phi_{\mathrm{y}}+\frac{\partial w_{0}}{\partial y}\right) \\
w(x, y, z, t)=w_{0}(x, y, t)
\end{gathered}
$$

where $c_{1}=4 /\left(3 h^{2}\right), u_{0}, v_{0}$, and $w_{0}$ are displacement components of middle plane corresponding to the coordinates $(x, y, z), \phi_{\mathrm{x}}$, and $\phi_{\mathrm{y}}$ are the rotations of the transverse normal in middle plane with respect to the $y$ and $x$ axes, respectively. The von Karman strain-displacement relation is obtained as 


$$
\begin{gathered}
\left\{\begin{array}{l}
\varepsilon_{\mathrm{x}} \\
\varepsilon_{\mathrm{y}} \\
\gamma_{\mathrm{xy}}
\end{array}\right\}=\left\{\begin{array}{l}
\varepsilon_{\mathrm{x}}^{(0)} \\
\varepsilon_{\mathrm{y}}^{(0)} \\
\gamma_{\mathrm{xy}}^{(0)}
\end{array}\right\}+z\left\{\begin{array}{l}
\varepsilon_{\mathrm{x}}^{(1)} \\
\varepsilon_{y}^{(1)} \\
\gamma_{\mathrm{xy}}^{(1)}
\end{array}\right\}+z^{3}\left\{\begin{array}{c}
\varepsilon_{\mathrm{x}}^{(3)} \\
\varepsilon_{y}^{(3)} \\
\gamma_{\mathrm{xy}}^{(3)}
\end{array}\right\} \\
\left\{\begin{array}{l}
\gamma_{\mathrm{xy}} \\
\gamma_{\mathrm{xz}}
\end{array}\right\}=\left\{\begin{array}{l}
\gamma_{\mathrm{yz}}^{(0)} \\
\gamma_{\mathrm{xz}}^{(0)}
\end{array}\right\}+z^{2}\left\{\begin{array}{l}
\gamma_{\mathrm{yz}}^{(2)} \\
\gamma_{\mathrm{xz}}^{(2)}
\end{array}\right\}
\end{gathered}
$$

where

$$
\begin{gathered}
\left\{\begin{array}{l}
\varepsilon_{\mathrm{x}}^{(0)} \\
\varepsilon_{\mathrm{y}}^{(0)} \\
\gamma_{\mathrm{xy}}^{(0)}
\end{array}\right\}=\left\{\begin{array}{c}
\frac{\partial u_{0}}{\partial x}+\frac{1}{2}\left(\frac{\partial w_{0}}{\partial x}\right)^{2} \\
\frac{\partial v_{0}}{\partial x}+\frac{1}{2}\left(\frac{\partial w_{0}}{\partial y}\right)^{2} \\
\frac{\partial u_{0}}{\partial y}+\frac{\partial v_{0}}{\partial x}+\frac{\partial w_{0}}{\partial x} \frac{\partial w_{0}}{\partial y}
\end{array}\right\} \\
\left\{\begin{array}{l}
\varepsilon_{\mathrm{x}}^{(1)} \\
\varepsilon_{\mathrm{y}}^{(1)} \\
\gamma_{\mathrm{xy}}^{(1)}
\end{array}\right\}=\left\{\begin{array}{c}
\frac{\partial \phi_{\mathrm{x}}}{\partial x} \\
\frac{\partial \phi_{\mathrm{y}}}{\partial y} \\
\frac{\partial \phi_{\mathrm{x}}}{\partial y}+\frac{\partial \phi_{\mathrm{y}}}{\partial x}
\end{array}\right\},\left\{\begin{array}{l}
\varepsilon_{\mathrm{x}}^{(3)} \\
\varepsilon_{\mathrm{y}}^{(3)} \\
\gamma_{\mathrm{xy}}^{(3)}
\end{array}\right\}=-c_{1}\left\{\begin{array}{c}
\frac{\partial \phi_{\mathrm{x}}}{\partial x}+\frac{\partial^{2} w_{0}}{\partial x^{2}} \\
\frac{\partial \phi_{\mathrm{y}}}{\partial y}+\frac{\partial^{2} w_{0}}{\partial y^{2}} \\
\frac{\partial \phi_{\mathrm{x}}}{\partial y}+\frac{\partial \phi_{\mathrm{y}}}{\partial x}+2 \frac{\partial^{2} w_{0}}{\partial x \partial y}
\end{array}\right\} \\
\left\{\begin{array}{l}
\gamma_{\mathrm{yz}}^{(0)} \\
\gamma_{\mathrm{xz}}^{(0)}
\end{array}\right\}=\left\{\begin{array}{l}
\phi_{\mathrm{y}}+\frac{\partial w_{0}}{\partial y} \\
\phi_{\mathrm{x}}+\frac{\partial w_{0}}{\partial x}
\end{array}\right\},\left\{\begin{array}{l}
\gamma_{\mathrm{yz}}^{(2)} \\
\gamma_{\mathrm{xz}}^{(2)}
\end{array}\right\}=-3 c_{1}\left\{\begin{array}{c}
\phi_{\mathrm{y}}+\frac{\partial w_{0}}{\partial y} \\
\phi_{\mathrm{x}}+\frac{\partial w_{0}}{\partial x}
\end{array}\right\}
\end{gathered}
$$

The constitutive relations considering the thermal effects are written as

$$
\left\{\begin{array}{l}
\sigma_{\mathrm{x}} \\
\sigma_{\mathrm{y}} \\
\tau_{\mathrm{xy}} \\
\tau_{\mathrm{yz}} \\
\tau_{\mathrm{xz}}
\end{array}\right\}=\left\{\begin{array}{ccccc}
C_{11} & C_{12} & 0 & 0 & 0 \\
C_{21} & C_{22} & 0 & 0 & 0 \\
0 & 0 & C_{66} & 0 & 0 \\
0 & 0 & 0 & C_{44} & 0 \\
0 & 0 & 0 & 0 & C_{55}
\end{array}\right\}\left\{\left\{\begin{array}{c}
\varepsilon_{\mathrm{x}} \\
\varepsilon_{\mathrm{y}} \\
\gamma_{\mathrm{xy}} \\
\gamma_{\mathrm{yz}} \\
\gamma_{\mathrm{xz}}
\end{array}\right\}-\left\{\begin{array}{c}
\alpha_{\mathrm{x}}^{\text {composite }} \\
\alpha_{\mathrm{y}}^{\text {composite }} \\
0 \\
0 \\
0
\end{array}\right\} \Delta T\right\}
$$

where

$$
C_{11}=\frac{E_{\mathrm{x}}^{\text {composite }}}{1-v_{\mathrm{xy}}^{\text {composite }} v_{\mathrm{yx}}^{\text {composite }}}, \quad C_{22}=\frac{E_{\mathrm{y}}^{\text {composite }}}{1-v_{\mathrm{xy}}^{\text {composite }} v_{\mathrm{yx}}^{\text {composite }}}, \quad C_{12}=\frac{v_{\mathrm{yx}}^{\text {composite }} E_{\mathrm{x}}^{\text {composite }}}{1-v_{\mathrm{xy}}^{\text {composite }} v_{\mathrm{yx}}^{\text {composite }}}=C_{21} \text {, }
$$




$$
C_{44}=G_{\mathrm{yz}}^{\text {composite }}, C_{55}=G_{\mathrm{xz}}^{\text {composite }}, C_{66}=G_{\mathrm{xy}}^{\text {composite }}
$$

In addition, we assume that $G_{\mathrm{yz}}^{\text {composite }}=1.2 G_{\mathrm{xy}}^{\text {composite }}=1.2 G_{\mathrm{xz}}^{\text {composite }}[18]$. By applying Hamilton's principle

$$
\int_{t_{1}}^{t_{2}}(\delta T-\delta U+\delta W) d t=0
$$

where $\delta T, \delta U$, and $\delta W$ is the kinetic energy, potential energy, and external work raised from the virtual displacement with

$$
\begin{aligned}
& \delta T=\int_{\Omega} \int_{-\frac{h}{2}}^{\frac{h}{2}} \rho_{0}(\dot{u} \delta \dot{u}+\dot{v} \delta \dot{v}+\dot{w} \delta \dot{w}) \mathrm{dxdydz} \\
& =\int_{\Omega} \int_{-\frac{h}{2}}^{\frac{h}{2}} \rho_{0}\left[\left(\dot{u}_{0}+z \dot{\phi}_{\mathrm{x}}-c_{1} z^{3} \dot{\varphi}_{\mathrm{x}}\right)\left(\delta \dot{u}_{0}+z \delta \dot{\phi}_{\mathrm{x}}-c_{1} z^{3} \delta \dot{\varphi}_{\mathrm{x}}\right)+\left(\dot{v}_{0}+z \dot{\phi}_{\mathrm{y}}-c_{1} z^{3} \dot{\varphi}_{\mathrm{y}}\right)\left(\delta \dot{v}_{0}+z \delta \dot{\phi}_{\mathrm{y}}\right.\right. \\
& \left.\left.-c_{1} z^{3} \delta \dot{\varphi}_{\mathrm{y}}\right)+\dot{w}_{0} \delta \dot{w}_{0}\right] \mathrm{dzdxdy} \\
& =\int_{\Omega}\left[\left(I_{0} \dot{u}_{0}+I_{1} \dot{\phi}_{\mathrm{x}}-c_{1} I_{3} \dot{\varphi}_{\mathrm{x}}\right) \delta \dot{u}_{0}+\left(I_{1} \dot{u}_{0}+I_{2} \dot{\phi}_{\mathrm{x}}-c_{1} I_{4} \dot{\varphi}_{\mathrm{x}}\right) \delta \dot{\phi}_{\mathrm{x}}-c_{1}\left(I_{3} \dot{u}_{0}+I_{4} \dot{\phi}_{\mathrm{x}}-c_{1} I_{6} \dot{\varphi}_{\mathrm{x}}\right) \delta \dot{\varphi}_{\mathrm{x}}\right. \\
& +\left(I_{0} \dot{v}_{0}+I_{1} \dot{\phi}_{\mathrm{y}}-c_{1} I_{3} \dot{\varphi}_{\mathrm{y}}\right) \delta \dot{v}_{0}+\left(I_{1} \dot{v}_{0}+I_{2} \dot{\phi}_{\mathrm{y}}-c_{1} I_{4} \dot{\varphi}_{\mathrm{y}}\right) \delta \dot{\phi}_{\mathrm{y}}-c_{1}\left(I_{3} \dot{u}_{0}+I_{4} \dot{\phi}_{\mathrm{y}}-c_{1} I_{6} \dot{\varphi}_{\mathrm{y}}\right) \delta \dot{\varphi}_{\mathrm{y}} \\
& \left.+I_{0} w_{0} \delta w_{0}\right] \mathrm{dxdy} \\
& =\int_{\Omega}\left[I_{0}\left(-\ddot{u}_{0} \delta u_{0}-\ddot{v}_{0} \delta v_{0}-\ddot{w}_{0} \delta w_{0}\right)+I_{1}\left(-\ddot{u}_{0} \delta \phi_{\mathrm{x}}-\ddot{v}_{0} \delta \phi_{\mathrm{y}}-\ddot{\phi}_{\mathrm{x}} \delta u_{0}-\ddot{\phi}_{\mathrm{y}} \delta v_{0}\right)+I_{2}\left(-\ddot{\phi}_{\mathrm{x}} \delta \phi_{\mathrm{x}}-\ddot{\phi}_{\mathrm{y}} \delta \phi_{\mathrm{y}}\right)\right. \\
& +I_{3}\left(c_{1} \ddot{u}_{0} \delta \phi_{\mathrm{x}}+c_{1} \ddot{v}_{0} \delta \phi_{\mathrm{y}}+c_{1} \ddot{\phi}_{x} \delta u_{0}+c_{1} \ddot{\phi}_{y} \delta v_{0}+c_{1} \ddot{w}_{0, \mathrm{x}} \delta u_{0}+c_{1} \ddot{w}_{0, \mathrm{y}} \delta v_{0}-c_{1} \delta w_{0} \ddot{u}_{0, \mathrm{x}}-c_{1} \ddot{v}_{0, \mathrm{y}} \delta w_{0}\right) \\
& +I_{4}\left(2 c_{1} \ddot{\phi}_{\mathrm{x}} \delta \phi_{\mathrm{x}}+2 c_{1} \ddot{\phi}_{\mathrm{y}} \delta \phi_{\mathrm{y}}-c_{1} \ddot{\phi}_{\mathrm{x}, \mathrm{x}} \delta w_{0}-c_{1} \ddot{\phi}_{\mathrm{y}, \mathrm{y}} \delta w_{0}+c_{1} \delta \phi_{\mathrm{x}} \ddot{w}_{0, \mathrm{x}}+c_{1} \ddot{w}_{0, \mathrm{y}} \delta \phi_{\mathrm{y}}\right) \\
& +I_{6}\left(-c_{1}^{2} \ddot{\phi}_{\mathrm{x}} \delta \phi_{\mathrm{x}}-c_{1}^{2} \ddot{\phi}_{\mathrm{y}} \delta \phi_{\mathrm{y}}+c_{1}^{2} \ddot{\phi}_{\mathrm{x}, \mathrm{x}} \delta w_{0}+c_{1}^{2} \ddot{\phi}_{\mathrm{y}, \mathrm{y}} \delta w_{0}-c_{1}^{2} \ddot{w}_{0, \mathrm{x}} \delta \phi_{\mathrm{x}}-c_{1}^{2} \ddot{w}_{0, \mathrm{y}} \delta \phi_{\mathrm{y}}+c_{1}^{2} \ddot{w}_{0, \mathrm{xx}} \delta w_{0}\right. \\
& \left.+c_{1}^{2} \ddot{w}_{0, \mathrm{yy}} \delta w_{0}\right) \mathrm{dxdy} \\
& \delta U=\int_{\Omega} \int_{-\frac{h}{2}}^{\frac{h}{2}}\left(\sigma_{\mathrm{x}} \delta \varepsilon_{\mathrm{x}}+\sigma_{\mathrm{y}} \delta \varepsilon_{\mathrm{y}}+\tau_{\mathrm{xy}} \delta \gamma_{\mathrm{xy}}+\tau_{\mathrm{yz}} \delta \gamma_{\mathrm{yz}}+\tau_{\mathrm{xz}} \delta \gamma_{\mathrm{xz}}\right) \mathrm{dxdydz} \\
& =\int_{\Omega} \int_{-\frac{h}{2}}^{\frac{h}{2}}\left[\sigma_{\mathrm{x}}\left(\delta \varepsilon_{\mathrm{x}}^{(0)}+z \delta \varepsilon_{\mathrm{x}}^{(1)}-c_{1} z^{3} \delta \varepsilon_{\mathrm{x}}^{(3)}\right)+\sigma_{\mathrm{y}}\left(\delta \varepsilon_{\mathrm{y}}^{(0)}+z \delta \varepsilon_{\mathrm{y}}^{(1)}-c_{1} z^{3} \delta \varepsilon_{\mathrm{y}}^{(3)}\right)+\tau_{\mathrm{xy}}\left(\delta \gamma_{\mathrm{xy}}^{(0)}+z \delta \gamma_{\mathrm{xy}}^{(1)}-c_{1} z^{3} \delta \gamma_{\mathrm{xy}}^{(3)}\right)\right. \\
& \left.+\tau_{\mathrm{xz}}\left(\delta \gamma_{\mathrm{xz}}^{(0)}+z^{2} \delta \gamma_{\mathrm{xz}}^{(2)}\right)+\tau_{\mathrm{yz}}\left(\delta \gamma_{\mathrm{yz}}^{(0)}+z^{2} \delta \gamma_{\mathrm{yz}}^{(2)}\right)\right] \mathrm{dzdxdy} \\
& =\int_{\Omega}\left(N_{\mathrm{x}} \delta \varepsilon_{\mathrm{x}}^{(0)}+M_{\mathrm{x}} \delta \varepsilon_{\mathrm{x}}^{(1)}-c_{1} P_{\mathrm{x}} \delta \varepsilon_{\mathrm{x}}^{(3)}+N_{\mathrm{y}} \delta \varepsilon_{\mathrm{y}}^{(0)}+M_{\mathrm{y}} \delta \varepsilon_{\mathrm{y}}^{(1)}-c_{1} P_{\mathrm{y}} \delta \varepsilon_{\mathrm{y}}^{(3)}+N_{\mathrm{xy}} \delta \gamma_{\mathrm{xy}}^{(0)}+M_{\mathrm{xy}} \delta \gamma_{\mathrm{xy}}^{(1)}-c_{1} P_{\mathrm{xy}} \delta \varepsilon_{\mathrm{xy}}^{(3)}\right.
\end{aligned}
$$




$$
\begin{aligned}
& \left.\quad+Q_{\mathrm{x}} \delta \gamma_{\mathrm{xz}}^{(0)}+R_{\mathrm{x}} \delta \gamma_{\mathrm{xz}}^{(2)}+Q_{\mathrm{y}} \delta \gamma_{\mathrm{yz}}^{(0)}+R_{\mathrm{y}} \delta \gamma_{\mathrm{yz}}^{(2)}\right) \mathrm{dxdy} \\
& =\int_{\Omega}\left(3 c_{1} R_{\mathrm{yz}, \mathrm{y}} \delta w_{0}-R_{\mathrm{yz}} 3 c_{1} \delta \phi_{\mathrm{y}}+c_{2} R_{\mathrm{xz}, \mathrm{x}} \delta w_{0}-Q_{\mathrm{y} z, \mathrm{y}} \delta w_{0}+Q_{\mathrm{yz}} \delta \phi_{\mathrm{y}}-Q_{\mathrm{xz}, \mathrm{x}} \delta w_{0}-R_{\mathrm{xz}} 3 c_{1} \delta \phi_{\mathrm{x}}-N_{\mathrm{xy}, \mathrm{x}} w_{0, \mathrm{y}} \delta w_{0}\right. \\
& +c_{1} P_{\mathrm{xy}, \mathrm{x}} \delta \phi_{\mathrm{y}}-2 c_{1} P_{\mathrm{xy}, \mathrm{xy}} \delta w_{0}+c_{1} P_{\mathrm{xy}, \mathrm{y}} \delta \phi_{\mathrm{x}}-2 N_{\mathrm{xy}} w_{0, \mathrm{xy}} \delta w_{0}-N_{\mathrm{xy}, \mathrm{y}} w_{0, \mathrm{x}} \delta w_{0}+Q_{\mathrm{xz}} \delta \phi_{\mathrm{x}}-M_{\mathrm{xy}, \mathrm{y}} \delta \phi_{\mathrm{x}}-M_{\mathrm{xy}, \mathrm{x}} \delta \phi_{\mathrm{y}} \\
& -N_{\mathrm{xy}, \mathrm{y}} \delta u_{0}-N_{\mathrm{xy}, \mathrm{x}} \delta v_{0}-M_{\mathrm{y}, \mathrm{y}} \delta \phi_{\mathrm{y}}-N_{\mathrm{y}, \mathrm{y}} \delta v_{0}+c_{1} P_{\mathrm{y}, \mathrm{y}} \delta \phi_{\mathrm{y}}-c_{1} P_{\mathrm{y}, \mathrm{yy}} \delta w_{0}-N_{\mathrm{y}, \mathrm{y}} w_{0, \mathrm{y}} \delta w_{0}-N_{\mathrm{y}} w_{0, \mathrm{yy}} \delta w_{0} \\
& \left.-M_{\mathrm{x}, \mathrm{x}} \delta \phi_{\mathrm{x}}-N_{\mathrm{x}, \mathrm{x}} \delta u_{0}-N_{\mathrm{x}, \mathrm{x}} w_{0, \mathrm{x}} \delta w_{0}-N_{\mathrm{x}} w_{0, \mathrm{xx}} \delta w_{0}+c_{1} P_{\mathrm{x}, \mathrm{x}} \delta \phi_{\mathrm{x}}-c_{1} P_{\mathrm{x}, \mathrm{xx}} \delta w_{0}\right) \mathrm{dxdy}(15 \mathrm{~b}) \\
& \qquad W=\int_{\Omega}\left(q \delta w_{0}+P_{\mathrm{a}} \delta w_{0}-\mu \dot{w}_{0} \delta w_{0}\right) \mathrm{dxdy}
\end{aligned}
$$

The strain can be written in terms of the generalized displacements using Eq. (10).

Substituting Eq. (15) into Eq. (14), the equations of motion are obtained as follows

$$
\begin{gathered}
\frac{\partial N_{\mathrm{xy}}}{\partial y}+\frac{\partial N_{\mathrm{x}}}{\partial x}=I_{0} \ddot{u}_{0}+I_{1} \ddot{\phi}_{\mathrm{x}}-I_{3} c_{1} \ddot{\phi}_{\mathrm{x}}-I_{3} c_{1} \frac{\partial \ddot{w}_{0}}{\partial x} \\
\frac{\partial N_{\mathrm{xy}}}{\partial x}+\frac{\partial N_{\mathrm{y}}}{\partial y}=I_{0} \ddot{v}_{0}+I_{1} \ddot{\phi}_{\mathrm{y}}-I_{3} c_{1} \ddot{\phi}_{\mathrm{y}}-I_{3} c_{1} \frac{\partial \ddot{w}_{0}}{\partial y} \\
\frac{\partial Q_{\mathrm{xz}}}{\partial x}+\frac{\partial Q_{\mathrm{yz}}}{\partial y}-3 c_{1}\left(\frac{\partial R_{\mathrm{xz}}}{\partial x}+\frac{\partial R_{\mathrm{yz}}}{\partial y}\right)+\frac{\partial}{\partial x}\left(N_{\mathrm{x}} \frac{\partial w_{0}}{\partial x}+N_{\mathrm{xy}} \frac{\partial w_{0}}{\partial y}\right)+\frac{\partial}{\partial y}\left(N_{\mathrm{y}} \frac{\partial w_{0}}{\partial y}+N_{\mathrm{xy}} \frac{\partial w_{0}}{\partial x}\right) \\
+c_{1}\left(\frac{\partial^{2} P_{\mathrm{x}}}{\partial x^{2}}+2 \frac{\partial^{2} P_{\mathrm{xy}}}{\partial x \partial y}+\frac{\partial^{2} P_{\mathrm{y}}}{\partial y^{2}}\right)+q+P_{\mathrm{a}}-\mu \dot{w}_{0} \\
=I_{0} \ddot{w}_{0}+I_{3} c_{1} \frac{\partial \ddot{i}_{0}}{\partial x}+I_{3} c_{1} \frac{\partial \ddot{v}_{0}}{\partial y}+I_{4} c_{1} \frac{\partial \ddot{\phi}_{\mathrm{x}}}{\partial x}+I_{4} c_{1} \frac{\partial \ddot{\phi}_{\mathrm{y}}}{\partial y}-I_{6} c_{1}^{2} \frac{\partial \ddot{\phi}_{\mathrm{x}}}{\partial x}-I_{6} c_{1}^{2} \frac{\partial \ddot{\phi}_{\mathrm{y}}}{\partial y} \\
-I_{6} c_{1}^{2} \frac{\partial^{2} \ddot{w}_{0}}{\partial x^{2}}-I_{6} c_{1}^{2} \frac{\partial^{2} \ddot{w}_{0}}{\partial y^{2}} \\
\frac{\partial M_{\mathrm{y}}}{\partial y}+\frac{\partial M_{\mathrm{xy}}}{\partial x}-Q_{\mathrm{yz}}+3 c_{1} R_{\mathrm{yz}}-c_{1} \frac{\partial P_{\mathrm{y}}}{\partial y}-c_{1} \frac{\partial P_{\mathrm{xy}}}{\partial x}-Q_{\mathrm{xz}}+3 c_{1} R_{\mathrm{xz}}-c_{1} \frac{\partial P_{\mathrm{x}}}{\partial x}-c_{1} \frac{\partial P_{\mathrm{xy}}}{\partial y} \\
=I_{2} \ddot{\phi}_{\mathrm{x}}-I_{3} \ddot{u}_{1} \ddot{u}_{0}-I_{4} 2 c_{1} \ddot{\phi}_{\mathrm{x}}-I_{4} c_{1} \frac{\partial \ddot{w}_{0}}{\partial x}+I_{6} c_{1}^{2} \ddot{\phi}_{\mathrm{x}}+I_{6} c_{1}^{2} \frac{\partial \ddot{w}_{0}}{\partial x}
\end{gathered}
$$




$$
=I_{1} \ddot{v}_{0}+I_{2} \ddot{\phi}_{\mathrm{y}}-I_{3} c_{1} \ddot{v}_{0}-I_{4} 2 c_{1} \ddot{\phi}_{\mathrm{y}}-I_{4} c_{1} \frac{\partial \ddot{w}_{0}}{\partial y}+I_{6} c_{1}^{2} \ddot{\phi}_{\mathrm{y}}+I_{6} c_{1}^{2} \frac{\partial \ddot{w}_{0}}{\partial y}
$$

where

$$
I_{\mathrm{k}}=\int_{-\frac{\mathrm{h}}{2}}^{\frac{\mathrm{h}}{2}} \rho z^{\mathrm{k}} \mathrm{dz}(\mathrm{k}=0,1,2, \cdots, 6)
$$

The stress resultants are related to the strains by the relations

$$
\begin{gathered}
{\left[\begin{array}{c}
\boldsymbol{N} \\
\boldsymbol{M} \\
\boldsymbol{P}
\end{array}\right]=\left[\begin{array}{lll}
\boldsymbol{A} & \boldsymbol{B} & \boldsymbol{E} \\
\boldsymbol{B} & \boldsymbol{D} & \boldsymbol{F} \\
\boldsymbol{E} & \boldsymbol{F} & \boldsymbol{H}
\end{array}\right]\left[\begin{array}{l}
\boldsymbol{\varepsilon}^{(0)} \\
\boldsymbol{\varepsilon}^{(1)} \\
\boldsymbol{\varepsilon}^{(3)}
\end{array}\right]-\left[\begin{array}{c}
\boldsymbol{N}^{T} \\
\boldsymbol{M}^{T} \\
\boldsymbol{P}^{T}
\end{array}\right]} \\
{\left[\begin{array}{l}
\boldsymbol{Q} \\
\boldsymbol{R}
\end{array}\right]=\left[\begin{array}{ll}
\boldsymbol{A} & \boldsymbol{D} \\
\boldsymbol{D} & \boldsymbol{F}
\end{array}\right]\left[\begin{array}{l}
\boldsymbol{\gamma}^{(0)} \\
\boldsymbol{\gamma}^{(2)}
\end{array}\right]}
\end{gathered}
$$

where

$$
\begin{gathered}
\left(A_{\mathrm{ij}}, B_{\mathrm{ij}}, D_{\mathrm{ij}}, E_{\mathrm{ij}}, F_{\mathrm{ij}}, H_{\mathrm{ij}}\right)=\int_{-\frac{\mathrm{h}}{2}}^{\frac{\mathrm{h}}{2}} C_{\mathrm{ij}}\left(1, z, z^{2}, z^{3}, z^{4}, z^{6}\right) \mathrm{dz},(\mathrm{i}, \mathrm{j}=1,2,6) \\
\left(A_{\mathrm{ij}}, D_{\mathrm{ij}}, F_{\mathrm{ij}}\right)=\int_{-\frac{\mathrm{h}}{2}}^{\frac{\mathrm{h}}{2}} C_{\mathrm{ij}}\left(1, z^{2}, z^{4}\right) \mathrm{dz},(\mathrm{i}, \mathrm{j}=4,5)
\end{gathered}
$$

and the thermal stress resultants are represented as

$$
\begin{gathered}
N^{T}=\left[\begin{array}{c}
N_{\mathrm{x}}^{T} \\
N_{\mathrm{y}}^{T}
\end{array}\right]=\int_{-\frac{\mathrm{h}}{2}}^{\frac{\mathrm{h}}{2}}\left[\begin{array}{ll}
C_{11} & C_{12} \\
C_{12} & C_{22}
\end{array}\right]\left[\begin{array}{l}
\alpha_{\mathrm{x}} \\
\alpha_{\mathrm{y}}
\end{array}\right] \Delta T \mathrm{dz} \\
M^{T}=\left[\begin{array}{l}
M_{\mathrm{x}}^{T} \\
M_{\mathrm{y}}^{T}
\end{array}\right]=\int_{-\frac{\mathrm{h}}{2}}^{\frac{\mathrm{h}}{2}}\left[\begin{array}{ll}
C_{11} & C_{12} \\
C_{12} & C_{22}
\end{array}\right]\left[\begin{array}{l}
\alpha_{\mathrm{x}} \\
\alpha_{\mathrm{y}}
\end{array}\right] \Delta T z \mathrm{dz} \\
P^{T}=\left[\begin{array}{l}
P_{\mathrm{x}}^{T} \\
P_{\mathrm{y}}^{T}
\end{array}\right]=\int_{-\frac{\mathrm{h}}{2}}^{\frac{\mathrm{h}}{2}}\left[\begin{array}{ll}
C_{11} & C_{12} \\
C_{12} & C_{22}
\end{array}\right]\left[\begin{array}{l}
\alpha_{\mathrm{x}} \\
\alpha_{\mathrm{y}}
\end{array}\right] \Delta T z^{3} \mathrm{dz}
\end{gathered}
$$

Each term of Eqs. (16) is shown in Appendix. By combining Appendix and Eqs. (16$20)$, the nonlinear equations of motion in terms of generalized displacements $\left(u_{0}, v_{0}, w_{0}\right.$. $\varphi_{\mathrm{x}}$ and $\left.\varphi_{\mathrm{y}}\right)$ are

$$
A_{16}\left(\frac{\partial w_{0}}{\partial x} \frac{\partial^{2} w_{0}}{\partial x \partial y}+\frac{\partial^{2} u_{0}}{\partial x \partial y}\right)+A_{26}\left(\frac{\partial w_{0}}{\partial y} \frac{\partial^{2} w_{0}}{\partial y^{2}}+\frac{\partial^{2} v_{0}}{\partial y^{2}}\right)+A_{66}\left(\frac{\partial w_{0}}{\partial x} \frac{\partial^{2} w_{0}}{\partial y^{2}}+\frac{\partial^{2} w_{0}}{\partial x \partial y} \frac{\partial w_{0}}{\partial y}+\frac{\partial^{2} u_{0}}{\partial y^{2}}+\frac{\partial^{2} v_{0}}{\partial x \partial y}\right)
$$




$$
\begin{aligned}
& +B_{16} \frac{\partial^{2} \phi_{\mathrm{x}}}{\partial x \partial y}+B_{26} \frac{\partial^{2} \phi_{\mathrm{y}}}{\partial y^{2}}+B_{66}\left(\frac{\partial^{2} \phi_{\mathrm{y}}}{\partial x \partial y}+\frac{\partial^{2} \phi_{\mathrm{x}}}{\partial y^{2}}\right)-c_{1} E_{16}\left(\frac{\partial^{2} \phi_{\mathrm{x}}}{\partial x \partial y}+\frac{\partial^{3} w_{0}}{\partial x^{2} \partial y}\right)-c_{1} E_{26}\left(\frac{\partial^{2} \phi_{\mathrm{y}}}{\partial y^{2}}+\frac{\partial^{3} w_{0}}{\partial y^{3}}\right) \\
& -c_{1} E_{66}\left(\frac{\partial^{2} \phi_{\mathrm{y}}}{\partial x^{2}}+\frac{\partial^{2} \phi_{\mathrm{x}}}{\partial x \partial y}+2 \frac{\partial^{3} w_{0}}{\partial x^{2} \partial y}\right)+A_{11}\left(\frac{\partial w_{0}}{\partial x} \frac{\partial^{2} w_{0}}{\partial x^{2}}+\frac{\partial^{2} u_{0}}{\partial x^{2}}\right)+A_{12}\left(\frac{\partial^{2} w_{0}}{\partial x \partial y} \frac{\partial w_{0}}{\partial y}+\frac{\partial^{2} v_{0}}{\partial x \partial y}\right) \\
& +A_{16}\left(\frac{\partial w_{0}}{\partial x} \frac{\partial^{2} w_{0}}{\partial x \partial y}+\frac{\partial^{2} w_{0}}{\partial x^{2}} \frac{\partial w_{0}}{\partial y}+\frac{\partial^{2} u_{0}}{\partial x \partial y}+\frac{\partial^{2} v_{0}}{\partial x^{2}}\right)+B_{11} \frac{\partial^{2} \phi_{\mathrm{x}}}{\partial x^{2}}+B_{12} \frac{\partial^{2} \phi_{\mathrm{y}}}{\partial x \partial y}+B_{16}\left(\frac{\partial^{2} \phi_{\mathrm{y}}}{\partial x^{2}}+\frac{\partial^{2} \phi_{\mathrm{x}}}{\partial x \partial y}\right) \\
& -c_{1} E_{11}\left(\frac{\partial^{2} \phi_{\mathrm{x}}}{\partial x^{2}}+\frac{\partial^{3} w_{0}}{\partial x^{3}}\right)-c_{1} E_{12}\left(\frac{\partial^{2} \phi_{\mathrm{y}}}{\partial x \partial y}+\frac{\partial^{3} w_{0}}{\partial x \partial y^{2}}\right)-c_{1} E_{16}\left(\frac{\partial^{2} \phi_{\mathrm{y}}}{\partial x^{2}}+\frac{\partial^{2} \phi_{\mathrm{x}}}{\partial x \partial y}+2 \frac{\partial^{3} w_{0}}{\partial x^{2} \partial y}\right)-\frac{\partial N_{\mathrm{x}}^{T}}{\partial x} \\
& =I_{0} \ddot{u}_{0}+I_{1} \ddot{\phi}_{\mathrm{x}}-I_{3} c_{1} \ddot{\phi}_{\mathrm{x}}-I_{3} c_{1} \frac{\partial \ddot{w}_{0}}{\partial x} \\
& A_{16}\left(\frac{\partial w_{0}}{\partial x} \frac{\partial^{2} w_{0}}{\partial x^{2}}+\frac{\partial^{2} u_{0}}{\partial x^{2}}\right)+A_{26}\left(\frac{\partial w_{0}}{\partial y} \frac{\partial^{2} w_{0}}{\partial x \partial y}+\frac{\partial^{2} v_{0}}{\partial x \partial y}\right)+A_{66}\left(\frac{\partial w_{0}}{\partial x} \frac{\partial^{2} w_{0}}{\partial x \partial y}+\frac{\partial^{2} w_{0}}{\partial x^{2}} \frac{\partial w_{0}}{\partial y}+\frac{\partial^{2} u_{0}}{\partial x \partial y}+\frac{\partial^{2} v_{0}}{\partial x^{2}}\right) \\
& +B_{16} \frac{\partial^{2} \phi_{\mathrm{x}}}{\partial x^{2}}+B_{26} \frac{\partial^{2} \phi_{\mathrm{y}}}{\partial x \partial y}+B_{66}\left(\frac{\partial^{2} \phi_{\mathrm{y}}}{\partial x^{2}}+\frac{\partial^{2} \phi_{\mathrm{x}}}{\partial x \partial y}\right)-c_{1} E_{16}\left(\frac{\partial^{2} \phi_{\mathrm{x}}}{\partial x^{2}}+\frac{\partial^{3} w_{0}}{\partial x^{3}}\right)-c_{1} E_{26}\left(\frac{\partial^{2} \phi_{\mathrm{y}}}{\partial x \partial y}+\frac{\partial^{3} w_{0}}{\partial x \partial y^{2}}\right) \\
& -c_{1} E_{66}\left(\frac{\partial^{2} \phi_{\mathrm{y}}}{\partial x^{2}}+\frac{\partial^{2} \phi_{\mathrm{x}}}{\partial x \partial y}+2 \frac{\partial^{3} w_{0}}{\partial x^{2} \partial y}\right)+A_{12}\left(\frac{\partial w_{0}}{\partial x} \frac{\partial^{2} w_{0}}{\partial x \partial y}+\frac{\partial^{2} u_{0}}{\partial x \partial y}\right)+A_{22}\left(\frac{\partial^{2} w_{0}}{\partial y^{2}} \frac{\partial w_{0}}{\partial y}+\frac{\partial^{2} v_{0}}{\partial y^{2}}\right) \\
& +A_{26}\left(\frac{\partial w_{0}}{\partial x} \frac{\partial^{2} w_{0}}{\partial y^{2}}+\frac{\partial^{2} w_{0}}{\partial x \partial y} \frac{\partial w_{0}}{\partial y}+\frac{\partial^{2} u_{0}}{\partial y^{2}}+\frac{\partial^{2} v_{0}}{\partial x \partial y}\right)+B_{12} \frac{\partial^{2} \phi_{\mathrm{x}}}{\partial x \partial y}+B_{22} \frac{\partial^{2} \phi_{\mathrm{y}}}{\partial y^{2}}+B_{26}\left(\frac{\partial^{2} \phi_{\mathrm{y}}}{\partial x \partial y}+\frac{\partial^{2} \phi_{\mathrm{x}}}{\partial y^{2}}\right) \\
& -c_{1} E_{12}\left(\frac{\partial^{2} \phi_{\mathrm{x}}}{\partial x \partial y}+\frac{\partial^{3} w_{0}}{\partial x^{2} \partial y}\right)-c_{1} E_{22}\left(\frac{\partial^{2} \phi_{\mathrm{y}}}{\partial y^{2}}+\frac{\partial^{3} w_{0}}{\partial y^{3}}\right)-c_{1} E_{26}\left(\frac{\partial^{2} \phi_{\mathrm{y}}}{\partial x \partial y}+\frac{\partial^{2} \phi_{\mathrm{x}}}{\partial y^{2}}+2 \frac{\partial^{3} w_{0}}{\partial x \partial y^{2}}\right)-\frac{\partial N_{\mathrm{y}}^{T}}{\partial y} \\
& =I_{0} \ddot{v}_{0}+I_{1} \ddot{\phi}_{\mathrm{y}}-I_{3} c_{1} \ddot{\phi}_{\mathrm{y}}-I_{3} c_{1} \frac{\partial \ddot{w}_{0}}{\partial y} \\
& A_{55}\left(\phi_{\mathrm{x}}+\frac{\partial w_{0}}{\partial x}\right)-c_{2} D_{55}\left(\phi_{\mathrm{x}}+\frac{\partial w_{0}}{\partial x}\right)-c_{2} D_{55}\left(\phi_{\mathrm{x}}+\frac{\partial w_{0}}{\partial x}\right)-c_{2}^{2} F_{55}\left(\phi_{\mathrm{x}}+\frac{\partial w_{0}}{\partial x}\right)+c_{1} E_{11}\left(\frac{\partial w_{0}}{\partial x} \frac{\partial^{2} w_{0}}{\partial x^{2}}+\frac{\partial^{2} u_{0}}{\partial x^{2}}\right) \\
& +c_{1} E_{12}\left(\frac{\partial^{2} w_{0}}{\partial x \partial y} \frac{\partial w_{0}}{\partial y}+\frac{\partial^{2} v_{0}}{\partial x \partial y}\right)+c_{1} E_{16}\left(\frac{\partial w_{0}}{\partial x} \frac{\partial^{2} w_{0}}{\partial x \partial y}+\frac{\partial^{2} w_{0}}{\partial x^{2}} \frac{\partial w_{0}}{\partial y}+\frac{\partial^{2} u_{0}}{\partial x \partial y}+\frac{\partial^{2} v_{0}}{\partial x^{2}}\right)+c_{1} F_{11} \frac{\partial^{2} \phi_{\mathrm{x}}}{\partial x^{2}}+c_{1} F_{12} \frac{\partial^{2} \phi_{\mathrm{y}}}{\partial x \partial y} \\
& +c_{1} F_{16}\left(\frac{\partial^{2} \phi_{\mathrm{y}}}{\partial x^{2}}+\frac{\partial^{2} \phi_{\mathrm{x}}}{\partial x \partial y}\right)-c_{1}^{2} H_{11}\left(\frac{\partial^{2} \phi_{\mathrm{x}}}{\partial x^{2}}+\frac{\partial^{3} w_{0}}{\partial x^{3}}\right)-c_{1}^{2} H_{12}\left(\frac{\partial^{2} \phi_{\mathrm{y}}}{\partial x \partial y}+\frac{\partial^{3} w_{0}}{\partial x \partial y^{2}}\right)-c_{1}^{2} H_{16}\left(\frac{\partial^{2} \phi_{\mathrm{y}}}{\partial x^{2}}+\frac{\partial^{2} \phi_{\mathrm{x}}}{\partial x \partial y}\right.
\end{aligned}
$$




$$
\begin{aligned}
& \left.+2 \frac{\partial^{3} w_{0}}{\partial x^{2} \partial y}\right)+c_{1} E_{16}\left(\frac{\partial w_{0}}{\partial x} \frac{\partial^{2} w_{0}}{\partial x \partial y}+\frac{\partial^{2} u_{0}}{\partial x \partial y}\right)+c_{1} E_{26}\left(\frac{\partial w_{0}}{\partial y} \frac{\partial^{2} w_{0}}{\partial y^{2}}+\frac{\partial^{2} v_{0}}{\partial y^{2}}\right)+c_{1} E_{66}\left(\frac{\partial w_{0}}{\partial x} \frac{\partial^{2} w_{0}}{\partial y^{2}}+\frac{\partial^{2} w_{0}}{\partial x \partial y} \frac{\partial w_{0}}{\partial y}\right. \\
& \left.+\frac{\partial^{2} u_{0}}{\partial y^{2}}+\frac{\partial^{2} v_{0}}{\partial x \partial y}\right)+c_{1} \frac{\partial P_{\mathrm{x}}^{T}}{\partial x}+c_{1} F_{16} \frac{\partial^{2} \phi_{\mathrm{x}}}{\partial x \partial y}+c_{1} F_{26} \frac{\partial^{2} \phi_{\mathrm{y}}}{\partial y^{2}}+c_{1} F_{66}\left(\frac{\partial^{2} \phi_{\mathrm{y}}}{\partial x \partial y}+\frac{\partial^{2} \phi_{\mathrm{x}}}{\partial y^{2}}\right)-c_{1}^{2} H_{16}\left(\frac{\partial^{2} \phi_{\mathrm{x}}}{\partial x \partial y}+\frac{\partial^{3} w_{0}}{\partial x^{2} \partial y}\right) \\
& -c_{1}^{2} H_{26}\left(\frac{\partial^{2} \phi_{\mathrm{y}}}{\partial y^{2}}+\frac{\partial^{3} w_{0}}{\partial y^{3}}\right)-c_{1}^{2} H_{66}\left(\frac{\partial^{2} \phi_{\mathrm{y}}}{\partial x \partial y}+\frac{\partial^{2} \phi_{\mathrm{x}}}{\partial y^{2}}+2 \frac{\partial^{3} w_{0}}{\partial x \partial y^{2}}\right)-D_{11} \frac{\partial^{2} \phi_{\mathrm{x}}}{\partial x^{2}}-D_{12} \frac{\partial^{2} \phi_{\mathrm{y}}}{\partial x \partial y}-D_{16}\left(\frac{\partial^{2} \phi_{\mathrm{y}}}{\partial x^{2}}+\frac{\partial^{2} \phi_{\mathrm{x}}}{\partial x \partial y}\right) \\
& +c_{1} F_{11}\left(\frac{\partial^{2} \phi_{\mathrm{x}}}{\partial x^{2}}+\frac{\partial^{3} w_{0}}{\partial x^{3}}\right)+c_{1} F_{12}\left(\frac{\partial^{2} \phi_{\mathrm{y}}}{\partial x \partial y}+\frac{\partial^{3} w_{0}}{\partial x \partial y^{2}}\right)+c_{1} F_{16}\left(\frac{\partial^{2} \phi_{\mathrm{y}}}{\partial x^{2}}+\frac{\partial^{2} \phi_{\mathrm{x}}}{\partial x \partial y}+2 \frac{\partial^{3} w_{0}}{\partial x^{2} \partial y}\right)-\frac{\partial M_{\mathrm{x}}^{T}}{\partial x} \\
& -B_{16}\left(\frac{\partial w_{0}}{\partial x} \frac{\partial^{2} w_{0}}{\partial x \partial y}+\frac{\partial^{2} u_{0}}{\partial x \partial y}\right)-B_{26}\left(\frac{\partial w_{0}}{\partial y} \frac{\partial^{2} w_{0}}{\partial y^{2}}+\frac{\partial^{2} v_{0}}{\partial y^{2}}\right)-B_{66}\left(\frac{\partial w_{0}}{\partial x} \frac{\partial^{2} w_{0}}{\partial y^{2}}+\frac{\partial^{2} w_{0}}{\partial x \partial y} \frac{\partial w_{0}}{\partial y}+\frac{\partial^{2} u_{0}}{\partial y^{2}}+\frac{\partial^{2} v_{0}}{\partial x \partial y}\right) \\
& -D_{16} \frac{\partial^{2} \phi_{\mathrm{x}}}{\partial x \partial y}-D_{26} \frac{\partial^{2} \phi_{\mathrm{y}}}{\partial y^{2}}-D_{66}\left(\frac{\partial^{2} \phi_{\mathrm{y}}}{\partial x \partial y}+\frac{\partial^{2} \phi_{\mathrm{x}}}{\partial y^{2}}\right)+c_{1} F_{16}\left(\frac{\partial^{2} \phi_{\mathrm{x}}}{\partial x \partial y}+\frac{\partial^{3} w_{0}}{\partial x^{2} \partial y}\right)+c_{1} F_{26}\left(\frac{\partial^{2} \phi_{\mathrm{y}}}{\partial y^{2}}+\frac{\partial^{3} w_{0}}{\partial y^{3}}\right) \\
& +c_{1} F_{66}\left(\frac{\partial^{2} \phi_{\mathrm{y}}}{\partial x \partial y}+\frac{\partial^{2} \phi_{\mathrm{x}}}{\partial y^{2}}+2 \frac{\partial^{3} w_{0}}{\partial x \partial y^{2}}\right) \\
& =I_{1} \ddot{u}_{0}+I_{2} \ddot{\phi}_{\mathrm{x}}-I_{3} c_{1} \ddot{u}_{0}-I_{4} 2 c_{1} \ddot{\phi}_{\mathrm{x}}-I_{4} c_{1} \frac{\partial \ddot{w}_{0}}{\partial x}+I_{6} c_{1}^{2} \ddot{\phi}_{\mathrm{x}}+I_{6} c_{1}^{2} \frac{\partial \ddot{w}_{0}}{\partial x} \\
& A_{44}\left(\phi_{\mathrm{y}}+\frac{\partial w_{0}}{\partial y}\right)-c_{2} D_{44}\left(\phi_{\mathrm{y}}+\frac{\partial w_{0}}{\partial y}\right)-c_{2} D_{44}\left(\phi_{\mathrm{y}}+\frac{\partial w_{0}}{\partial y}\right)+c_{2}^{2} F_{44}\left(\phi_{\mathrm{y}}+\frac{\partial w_{0}}{\partial y}\right)+c_{1} E_{12}\left(\frac{\partial w_{0}}{\partial x} \frac{\partial^{2} w_{0}}{\partial x \partial y}+\frac{\partial^{2} u_{0}}{\partial x \partial y}\right) \\
& +c_{1} E_{22}\left(\frac{\partial^{2} w_{0}}{\partial y^{2}} \frac{\partial w_{0}}{\partial y}+\frac{\partial^{2} v_{0}}{\partial y^{2}}\right)+c_{1} E_{26}\left(\frac{\partial w_{0}}{\partial x} \frac{\partial^{2} w_{0}}{\partial y^{2}}+\frac{\partial^{2} w_{0}}{\partial x \partial y} \frac{\partial w_{0}}{\partial y}+\frac{\partial^{2} u_{0}}{\partial y^{2}}+\frac{\partial^{2} v_{0}}{\partial x \partial y}\right)+c_{1} F_{12} \frac{\partial^{2} \phi_{\mathrm{x}}}{\partial x \partial y}+c_{1} F_{22} \frac{\partial^{2} \phi_{\mathrm{y}}}{\partial y^{2}} \\
& +c_{1} F_{26}\left(\frac{\partial^{2} \phi_{\mathrm{y}}}{\partial x \partial y}+\frac{\partial^{2} \phi_{\mathrm{x}}}{\partial y^{2}}\right)-c_{1}^{2} H_{12}\left(\frac{\partial^{2} \phi_{\mathrm{x}}}{\partial x \partial y}+\frac{\partial^{3} w_{0}}{\partial x^{2} \partial y}\right)-c_{1}^{2} H_{22}\left(\frac{\partial^{2} \phi_{\mathrm{y}}}{\partial y^{2}}+\frac{\partial^{3} w_{0}}{\partial y^{3}}\right)-c_{1}^{2} H_{26}\left(\frac{\partial^{2} \phi_{\mathrm{y}}}{\partial x \partial y}+\frac{\partial^{2} \phi_{\mathrm{x}}}{\partial y^{2}}\right. \\
& +2 \frac{\partial^{3} w_{0}}{\partial x \partial y^{2}}+c_{1} \frac{\partial P_{\mathrm{y}}^{T}}{\partial y}+c_{1} E_{16}\left(\frac{\partial w_{0}}{\partial x} \frac{\partial^{2} w_{0}}{\partial x^{2}}+\frac{\partial^{2} u_{0}}{\partial x^{2}}\right)+c_{1} E_{26}\left(\frac{\partial w_{0}}{\partial y} \frac{\partial^{2} w_{0}}{\partial x \partial y}+\frac{\partial^{2} v_{0}}{\partial x \partial y}\right) \\
& +c_{1} E_{66}\left(\frac{\partial w_{0}}{\partial x} \frac{\partial^{2} w_{0}}{\partial x \partial y}+\frac{\partial^{2} w_{0}}{\partial x^{2}} \frac{\partial w_{0}}{\partial y}+\frac{\partial^{2} u_{0}}{\partial x \partial y}+\frac{\partial^{2} v_{0}}{\partial x^{2}}\right)+c_{1} F_{16} \frac{\partial^{2} \phi_{\mathrm{x}}}{\partial x^{2}}+c_{1} F_{26} \frac{\partial^{2} \phi_{\mathrm{y}}}{\partial x \partial y}+c_{1} F_{66}\left(\frac{\partial^{2} \phi_{\mathrm{y}}}{\partial x^{2}}+\frac{\partial^{2} \phi_{\mathrm{x}}}{\partial x \partial y}\right) \\
& -c_{1}^{2} H_{16}\left(\frac{\partial^{2} \phi_{\mathrm{x}}}{\partial x^{2}}+\frac{\partial^{3} w_{0}}{\partial x^{3}}\right)-c_{1}^{2} H_{26}\left(\frac{\partial^{2} \phi_{\mathrm{y}}}{\partial x \partial y}+\frac{\partial^{3} w_{0}}{\partial x \partial y^{2}}\right)-c_{1}^{2} H_{66}\left(\frac{\partial^{2} \phi_{\mathrm{y}}}{\partial x^{2}}+\frac{\partial^{2} \phi_{\mathrm{x}}}{\partial x \partial y}+2 \frac{\partial^{3} w_{0}}{\partial x^{2} \partial y}\right)
\end{aligned}
$$




$$
\begin{aligned}
& -B_{12}\left(\frac{\partial w_{0}}{\partial x} \frac{\partial^{2} w_{0}}{\partial x \partial y}+\frac{\partial^{2} u_{0}}{\partial x \partial y}\right)-B_{22}\left(\frac{\partial^{2} w_{0}}{\partial y^{2}} \frac{\partial w_{0}}{\partial y}+\frac{\partial^{2} v_{0}}{\partial y^{2}}\right)-B_{26}\left(\frac{\partial w_{0}}{\partial x} \frac{\partial^{2} w_{0}}{\partial y^{2}}+\frac{\partial^{2} w_{0}}{\partial x \partial y} \frac{\partial w_{0}}{\partial y}+\frac{\partial^{2} u_{0}}{\partial y^{2}}+\frac{\partial^{2} v_{0}}{\partial x \partial y}\right) \\
& -D_{12} \frac{\partial^{2} \phi_{\mathrm{x}}}{\partial x \partial y}-D_{22} \frac{\partial^{2} \phi_{\mathrm{y}}}{\partial y^{2}}-D_{26}\left(\frac{\partial^{2} \phi_{\mathrm{y}}}{\partial x \partial y}+\frac{\partial^{2} \phi_{\mathrm{x}}}{\partial y^{2}}\right)+c_{1} F_{12}\left(\frac{\partial^{2} \phi_{\mathrm{x}}}{\partial x \partial y}+\frac{\partial^{3} w_{0}}{\partial x^{2} \partial y}\right)+c_{1} F_{22}\left(\frac{\partial^{2} \phi_{\mathrm{y}}}{\partial y^{2}}+\frac{\partial^{3} w_{0}}{\partial y^{3}}\right) \\
& +c_{1} F_{26}\left(\frac{\partial^{2} \phi_{\mathrm{y}}}{\partial x \partial y}+\frac{\partial^{2} \phi_{\mathrm{x}}}{\partial y^{2}}+2 \frac{\partial^{3} w_{0}}{\partial x \partial y^{2}}\right)-\frac{\partial M_{\mathrm{y}}^{T}}{\partial y}-B_{16}\left(\frac{\partial w_{0}}{\partial x} \frac{\partial^{2} w_{0}}{\partial x^{2}}+\frac{\partial^{2} u_{0}}{\partial x^{2}}\right)-B_{26}\left(\frac{\partial w_{0}}{\partial y} \frac{\partial^{2} w_{0}}{\partial x \partial y}+\frac{\partial^{2} v_{0}}{\partial x \partial y}\right) \\
& -B_{66}\left(\frac{\partial w_{0}}{\partial x} \frac{\partial^{2} w_{0}}{\partial x \partial y}+\frac{\partial^{2} w_{0}}{\partial x^{2}} \frac{\partial w_{0}}{\partial y}+\frac{\partial^{2} u_{0}}{\partial x \partial y}+\frac{\partial^{2} v_{0}}{\partial x^{2}}\right)-D_{16} \frac{\partial^{2} \phi_{\mathrm{x}}}{\partial x^{2}}-D_{26} \frac{\partial^{2} \phi_{\mathrm{y}}}{\partial x \partial y}-D_{66}\left(\frac{\partial^{2} \phi_{\mathrm{y}}}{\partial x^{2}}+\frac{\partial^{2} \phi_{\mathrm{x}}}{\partial x \partial y}\right) \\
& +c_{1} F_{16}\left(\frac{\partial^{2} \phi_{\mathrm{x}}}{\partial x^{2}}+\frac{\partial^{3} w_{0}}{\partial x^{3}}\right)+c_{1} F_{26}\left(\frac{\partial^{2} \phi_{\mathrm{y}}}{\partial x \partial y}+\frac{\partial^{3} w_{0}}{\partial x \partial y^{2}}\right)+c_{1} F_{66}\left(\frac{\partial^{2} \phi_{\mathrm{y}}}{\partial x^{2}}+\frac{\partial^{2} \phi_{\mathrm{x}}}{\partial x \partial y}+2 \frac{\partial^{3} w_{0}}{\partial x^{2} \partial y}\right) \\
& =I_{1} \ddot{v}_{0}+I_{2} \ddot{\phi}_{\mathrm{y}}-I_{3} c_{1} \ddot{v}_{0}-I_{4} 2 c_{1} \ddot{\phi}_{\mathrm{y}}-I_{4} c_{1} \frac{\partial \ddot{w}_{0}}{\partial y}+I_{6} c_{1}^{2} \ddot{\phi}_{\mathrm{y}}+I_{6} c_{1}^{2} \frac{\partial \ddot{w}_{0}}{\partial y} \\
& A_{55}\left(\frac{\partial \phi_{\mathrm{x}}}{\partial x}+\frac{\partial^{2} w_{0}}{\partial x^{2}}\right)-c_{2} D_{55}\left(\frac{\partial \phi_{\mathrm{x}}}{\partial x}+\frac{\partial^{2} w_{0}}{\partial x^{2}}\right)+A_{44}\left(\frac{\partial \phi_{\mathrm{y}}}{\partial y}+\frac{\partial^{2} w_{0}}{\partial y^{2}}\right)-c_{2} D_{44}\left(\frac{\partial \phi_{\mathrm{y}}}{\partial y}+\frac{\partial^{2} w_{0}}{\partial y^{2}}\right) \\
& +A_{11}\left(\left(\frac{\partial w_{0}}{\partial x}\right)^{2} \frac{\partial^{2} w_{0}}{\partial x^{2}}+\frac{\partial^{2} u_{0}}{\partial x^{2}} \frac{\partial w_{0}}{\partial x}\right)+A_{12}\left(\frac{\partial^{2} w_{0}}{\partial x \partial y} \frac{\partial w_{0}}{\partial x} \frac{\partial w_{0}}{\partial y}+\frac{\partial^{2} v_{0}}{\partial x \partial y} \frac{\partial w_{0}}{\partial x}\right) \\
& +A_{16}\left(\left(\frac{\partial w_{0}}{\partial x}\right)^{2} \frac{\partial^{2} w_{0}}{\partial x \partial y}+\frac{\partial^{2} w_{0}}{\partial x^{2}} \frac{\partial w_{0}}{\partial x} \frac{\partial w_{0}}{\partial y}+\frac{\partial^{2} u_{0}}{\partial x \partial y} \frac{\partial w_{0}}{\partial x}+\frac{\partial^{2} v_{0}}{\partial x^{2}} \frac{\partial w_{0}}{\partial x}\right) \\
& +B_{11} \frac{\partial^{2} \phi_{\mathrm{x}}}{\partial x^{2}} \frac{\partial w_{0}}{\partial x}+B_{12} \frac{\partial^{2} \phi_{\mathrm{y}}}{\partial x \partial y} \frac{\partial w_{0}}{\partial x}+B_{16}\left(\frac{\partial^{2} \phi_{\mathrm{y}}}{\partial x^{2}} \frac{\partial w_{0}}{\partial x}+\frac{\partial^{2} \phi_{\mathrm{x}}}{\partial x \partial y} \frac{\partial w_{0}}{\partial x}\right)-c_{1} E_{11}\left(\frac{\partial^{2} \phi_{\mathrm{x}}}{\partial x^{2}} \frac{\partial w_{0}}{\partial x}+\frac{\partial^{3} w_{0}}{\partial x^{3}} \frac{\partial w_{0}}{\partial x}\right) \\
& -c_{1} E_{12}\left(\frac{\partial^{2} \phi_{\mathrm{y}}}{\partial x \partial y} \frac{\partial w_{0}}{\partial x}+\frac{\partial^{3} w_{0}}{\partial x \partial y^{2}} \frac{\partial w_{0}}{\partial x}\right)-c_{1} E_{16}\left(\frac{\partial^{2} \phi_{\mathrm{y}}}{\partial x^{2}} \frac{\partial w_{0}}{\partial x}+\frac{\partial^{2} \phi_{\mathrm{x}}}{\partial x \partial y} \frac{\partial w_{0}}{\partial x}+2 \frac{\partial^{3} w_{0}}{\partial x^{2} \partial y} \frac{\partial w_{0}}{\partial x}\right)-\frac{\partial N_{\mathrm{x}}^{T}}{\partial x} \frac{\partial w_{0}}{\partial x} \\
& +A_{11}\left(\frac{1}{2}\left(\frac{\partial^{2} w_{0}}{\partial x^{2}}\right)^{2}+\frac{\partial u_{0}}{\partial x} \frac{\partial^{2} w_{0}}{\partial x^{2}}\right)+A_{12}\left(\frac{1}{2} \frac{\partial^{2} w_{0}}{\partial y^{2}} \frac{\partial^{2} w_{0}}{\partial x^{2}}+\frac{\partial v_{0}}{\partial y} \frac{\partial^{2} w_{0}}{\partial x^{2}}\right)+A_{16}\left(\left(\frac{\partial w_{0}}{\partial x}\right)^{2} \frac{\partial w_{0}}{\partial y}+\frac{\partial u_{0}}{\partial y} \frac{\partial^{2} w_{0}}{\partial x^{2}}\right) \\
& +\frac{\partial v_{0}}{\partial x} \frac{\partial^{2} w_{0}}{\partial x^{2}}+B_{11} \frac{\partial \phi_{\mathrm{x}}}{\partial x} \frac{\partial^{2} w_{0}}{\partial x^{2}}+B_{12} \frac{\partial \phi_{\mathrm{y}}}{\partial y} \frac{\partial^{2} w_{0}}{\partial x^{2}}+B_{16}\left(\frac{\partial \phi_{\mathrm{y}}}{\partial x} \frac{\partial^{2} w_{0}}{\partial x^{2}}+\frac{\partial \phi_{\mathrm{x}}}{\partial y} \frac{\partial^{2} w_{0}}{\partial x^{2}}\right)-c_{1} E_{11}\left(\frac{\partial \phi_{\mathrm{x}}}{\partial x} \frac{\partial^{2} w_{0}}{\partial x^{2}}+\left(\frac{\partial^{2} w_{0}}{\partial x^{2}}\right)^{2}\right) \\
& -c_{1} E_{12}\left(\frac{\partial \phi_{\mathrm{y}}}{\partial y} \frac{\partial^{2} w_{0}}{\partial x^{2}}+\frac{\partial^{2} w_{0}}{\partial y^{2}} \frac{\partial^{2} w_{0}}{\partial x^{2}}\right)-c_{1} E_{16}\left(\frac{\partial \phi_{\mathrm{y}}}{\partial x} \frac{\partial^{2} w_{0}}{\partial x^{2}}+\frac{\partial \phi_{\mathrm{x}}}{\partial y} \frac{\partial^{2} w_{0}}{\partial x^{2}}+2 \frac{\partial^{2} w_{0}}{\partial x \partial y} \frac{\partial^{2} w_{0}}{\partial x^{2}}\right)-N_{\mathrm{x}}^{T} \frac{\partial^{2} w_{0}}{\partial x^{2}}
\end{aligned}
$$




$$
\begin{aligned}
& +A_{16}\left(\frac{\partial w_{0}}{\partial x} \frac{\partial w_{0}}{\partial y} \frac{\partial^{2} w_{0}}{\partial x^{2}}+\frac{\partial^{2} u_{0}}{\partial x^{2}} \frac{\partial w_{0}}{\partial y}\right)+A_{26}\left(\left(\frac{\partial w_{0}}{\partial y}\right)^{2} \frac{\partial^{2} w_{0}}{\partial x \partial y}+\frac{\partial^{2} v_{0}}{\partial x \partial y} \frac{\partial w_{0}}{\partial y}\right) \\
& +A_{66}\left(\frac{\partial w_{0}}{\partial x} \frac{\partial w_{0}}{\partial y} \frac{\partial^{2} w_{0}}{\partial x \partial y}+\frac{\partial^{2} w_{0}}{\partial x^{2}}\left(\frac{\partial w_{0}}{\partial y}\right)^{2}+\frac{\partial^{2} u_{0}}{\partial x \partial y} \frac{\partial w_{0}}{\partial y}+\frac{\partial^{2} v_{0}}{\partial x^{2}} \frac{\partial w_{0}}{\partial y}\right)+B_{16} \frac{\partial^{2} \phi_{\mathrm{x}}}{\partial x^{2}} \frac{\partial w_{0}}{\partial y}+B_{26} \frac{\partial^{2} \phi_{\mathrm{y}}}{\partial x \partial y} \frac{\partial w_{0}}{\partial y} \\
& +B_{66}\left(\frac{\partial^{2} \phi_{\mathrm{y}}}{\partial x^{2}} \frac{\partial w_{0}}{\partial y}+\frac{\partial^{2} \phi_{\mathrm{x}}}{\partial x \partial y} \frac{\partial w_{0}}{\partial y}\right)-c_{1} E_{16}\left(\frac{\partial^{2} \phi_{\mathrm{x}}}{\partial x^{2}} \frac{\partial w_{0}}{\partial y}+\frac{\partial^{3} w_{0}}{\partial x^{3}} \frac{\partial w_{0}}{\partial y}\right)-c_{1} E_{26}\left(\frac{\partial^{2} \phi_{\mathrm{y}}}{\partial x \partial y} \frac{\partial w_{0}}{\partial y}+\frac{\partial^{3} w_{0}}{\partial x \partial y^{2}} \frac{\partial w_{0}}{\partial y}\right) \\
& -c_{1} E_{66}\left(\frac{\partial^{2} \phi_{\mathrm{y}}}{\partial x^{2}} \frac{\partial w_{0}}{\partial y}+\frac{\partial^{2} \phi_{\mathrm{x}}}{\partial x \partial y} \frac{\partial w_{0}}{\partial y}+2 \frac{\partial^{3} w_{0}}{\partial x^{2} \partial y} \frac{\partial w_{0}}{\partial y}\right)+A_{16}\left(\frac{1}{2} \frac{\partial^{2} w_{0}}{\partial x^{2}} \frac{\partial^{2} w_{0}}{\partial y^{2}}+\frac{\partial u_{0}}{\partial x} \frac{\partial^{2} w_{0}}{\partial y^{2}}\right) \\
& +A_{26}\left(\frac{1}{2}\left(\frac{\partial^{2} w_{0}}{\partial y^{2}}\right)^{2}+\frac{\partial v_{0}}{\partial y} \frac{\partial^{2} w_{0}}{\partial y^{2}}\right)+A_{66}\left(\frac{\partial w_{0}}{\partial x} \frac{\partial w_{0}}{\partial y} \frac{\partial^{2} w_{0}}{\partial y^{2}}+\frac{\partial u_{0}}{\partial y} \frac{\partial^{2} w_{0}}{\partial y^{2}}+\frac{\partial v_{0}}{\partial x} \frac{\partial^{2} w_{0}}{\partial y^{2}}\right)+B_{16} \frac{\partial \phi_{\mathrm{x}}}{\partial x} \frac{\partial^{2} w_{0}}{\partial y^{2}} \\
& +B_{26} \frac{\partial \phi_{\mathrm{y}}}{\partial y} \frac{\partial^{2} w_{0}}{\partial y^{2}}+B_{66}\left(\frac{\partial \phi_{\mathrm{y}}}{\partial x} \frac{\partial^{2} w_{0}}{\partial y^{2}}+\frac{\partial \phi_{\mathrm{x}}}{\partial y} \frac{\partial^{2} w_{0}}{\partial y^{2}}\right)-c_{1} E_{16}\left(\frac{\partial \phi_{\mathrm{x}}}{\partial x} \frac{\partial^{2} w_{0}}{\partial y^{2}}+\frac{\partial w_{0}}{\partial x^{2}} \frac{\partial^{2} w_{0}}{\partial y^{2}}\right)-c_{1} E_{26}\left(\frac{\partial \phi_{\mathrm{y}}}{\partial y} \frac{\partial^{2} w_{0}}{\partial y^{2}}\right. \\
& \left.+\left(\frac{\partial^{2} w_{0}}{\partial y^{2}}\right)^{2}\right)-c_{1} E_{66}\left(\frac{\partial \phi_{\mathrm{y}}}{\partial x} \frac{\partial^{2} w_{0}}{\partial y^{2}}+\frac{\partial \phi_{\mathrm{x}}}{\partial y} \frac{\partial^{2} w_{0}}{\partial y^{2}}+2 \frac{\partial^{2} w_{0}}{\partial x \partial y} \frac{\partial^{2} w_{0}}{\partial y^{2}}\right)+A_{12}\left(\frac{\partial w_{0}}{\partial x} \frac{\partial w_{0}}{\partial y} \frac{\partial^{2} w_{0}}{\partial x \partial y}+\frac{\partial^{2} u_{0}}{\partial x \partial y} \frac{\partial w_{0}}{\partial y}\right) \\
& +A_{22}\left(\frac{\partial^{2} w_{0}}{\partial y^{2}}\left(\frac{\partial w_{0}}{\partial y}\right)^{2}+\frac{\partial^{2} v_{0}}{\partial y^{2}} \frac{\partial w_{0}}{\partial y}\right)+A_{26}\left(\frac{\partial w_{0}}{\partial x} \frac{\partial w_{0}}{\partial y} \frac{\partial^{2} w_{0}}{\partial y^{2}}+\frac{\partial^{2} w_{0}}{\partial x \partial y}\left(\frac{\partial w_{0}}{\partial y}\right)^{2}+\frac{\partial^{2} u_{0}}{\partial y^{2}} \frac{\partial w_{0}}{\partial y}+\frac{\partial^{2} v_{0}}{\partial x \partial y} \frac{\partial w_{0}}{\partial y}\right) \\
& +B_{12} \frac{\partial^{2} \phi_{\mathrm{x}}}{\partial x \partial y} \frac{\partial w_{0}}{\partial y}+B_{22} \frac{\partial^{2} \phi_{\mathrm{y}}}{\partial y^{2}} \frac{\partial w_{0}}{\partial y}+B_{26}\left(\frac{\partial^{2} \phi_{\mathrm{y}}}{\partial x \partial y} \frac{\partial w_{0}}{\partial y}+\frac{\partial^{2} \phi_{\mathrm{x}}}{\partial y^{2}} \frac{\partial w_{0}}{\partial y}\right)-c_{1} E_{12}\left(\frac{\partial^{2} \phi_{\mathrm{x}}}{\partial x \partial y} \frac{\partial w_{0}}{\partial y}+\frac{\partial^{3} w_{0}}{\partial x^{2} \partial y} \frac{\partial w_{0}}{\partial y}\right) \\
& -c_{1} E_{22}\left(\frac{\partial^{2} \phi_{\mathrm{y}}}{\partial y^{2}} \frac{\partial w_{0}}{\partial y}+\frac{\partial^{3} w_{0}}{\partial y^{3}} \frac{\partial w_{0}}{\partial y}\right)-c_{1} E_{26}\left(\frac{\partial^{2} \phi_{\mathrm{y}}}{\partial x \partial y} \frac{\partial w_{0}}{\partial y}+\frac{\partial^{2} \phi_{\mathrm{x}}}{\partial y^{2}} \frac{\partial w_{0}}{\partial y}+2 \frac{\partial^{3} w_{0}}{\partial x \partial y^{2}} \frac{\partial w_{0}}{\partial y}\right)-\frac{\partial N_{\mathrm{y}}^{T}}{\partial y} \frac{\partial w_{0}}{\partial y} \\
& +A_{12}\left(\frac{1}{2} \frac{\partial^{2} w_{0}}{\partial x^{2}} \frac{\partial^{2} w_{0}}{\partial y^{2}}+\frac{\partial u_{0}}{\partial x} \frac{\partial^{2} w_{0}}{\partial y^{2}}\right)+A_{22}\left(\frac{1}{2}\left(\frac{\partial^{2} w_{0}}{\partial y^{2}}\right)^{2}+\frac{\partial v_{0}}{\partial y} \frac{\partial^{2} w_{0}}{\partial y^{2}}\right)+A_{26}\left(\frac{\partial w_{0}}{\partial x} \frac{\partial w_{0}}{\partial y} \frac{\partial^{2} w_{0}}{\partial y^{2}}+\frac{\partial u_{0}}{\partial y} \frac{\partial^{2} w_{0}}{\partial y^{2}}\right. \\
& \left.+\frac{\partial v_{0}}{\partial x} \frac{\partial^{2} w_{0}}{\partial y^{2}}\right)+B_{12} \frac{\partial \phi_{\mathrm{x}}}{\partial x} \frac{\partial^{2} w_{0}}{\partial y^{2}}+B_{22} \frac{\partial \phi_{\mathrm{y}}}{\partial y} \frac{\partial^{2} w_{0}}{\partial y^{2}}+B_{26}\left(\frac{\partial \phi_{\mathrm{y}}}{\partial x} \frac{\partial^{2} w_{0}}{\partial y^{2}}+\frac{\partial \phi_{\mathrm{x}}}{\partial y} \frac{\partial^{2} w_{0}}{\partial y^{2}}\right)-c_{1} E_{12}\left(\frac{\partial \phi_{\mathrm{x}}}{\partial x} \frac{\partial^{2} w_{0}}{\partial y^{2}}\right. \\
& \left.+\frac{\partial^{2} w_{0}}{\partial x^{2}} \frac{\partial^{2} w_{0}}{\partial y^{2}}\right)-c_{1} E_{22}\left(\frac{\partial \phi_{\mathrm{y}}}{\partial y} \frac{\partial^{2} w_{0}}{\partial y^{2}}+\left(\frac{\partial^{2} w_{0}}{\partial y^{2}}\right)^{2}\right)-c_{1} E_{26}\left(\frac{\partial \phi_{\mathrm{y}}}{\partial x} \frac{\partial^{2} w_{0}}{\partial y^{2}}+\frac{\partial \phi_{\mathrm{x}}}{\partial y} \frac{\partial^{2} w_{0}}{\partial y^{2}}+2 \frac{\partial^{2} w_{0}}{\partial x \partial y} \frac{\partial^{2} w_{0}}{\partial y^{2}}\right) \\
& -N_{\mathrm{y}}^{T} \frac{\partial^{2} w_{0}}{\partial y^{2}}+\frac{\partial N_{\mathrm{xy}}}{\partial y}
\end{aligned}
$$




$$
\begin{aligned}
& =A_{16}\left(\left(\frac{\partial w_{0}}{\partial x}\right)^{2} \frac{\partial^{2} w_{0}}{\partial x \partial y}+\frac{\partial^{2} u_{0}}{\partial x \partial y} \frac{\partial w_{0}}{\partial x}\right)+A_{26}\left(\frac{\partial w_{0}}{\partial x} \frac{\partial w_{0}}{\partial y} \frac{\partial^{2} w_{0}}{\partial y^{2}}+\frac{\partial^{2} v_{0}}{\partial y^{2}} \frac{\partial w_{0}}{\partial x}\right) \\
& +A_{66}\left(\left(\frac{\partial w_{0}}{\partial x}\right)^{2} \frac{\partial^{2} w_{0}}{\partial y^{2}}+\frac{\partial^{2} w_{0}}{\partial x \partial y} \frac{\partial w_{0}}{\partial x} \frac{\partial w_{0}}{\partial y}+\frac{\partial^{2} u_{0}}{\partial y^{2}} \frac{\partial w_{0}}{\partial x}+\frac{\partial^{2} v_{0}}{\partial x \partial y} \frac{\partial w_{0}}{\partial x}\right) \\
& +B_{16} \frac{\partial^{2} \phi_{\mathrm{x}}}{\partial x \partial y} \frac{\partial w_{0}}{\partial x}+B_{26} \frac{\partial^{2} \phi_{\mathrm{y}}}{\partial y^{2}} \frac{\partial w_{0}}{\partial x}+B_{66}\left(\frac{\partial^{2} \phi_{\mathrm{y}}}{\partial x \partial y} \frac{\partial w_{0}}{\partial x}+\frac{\partial^{2} \phi_{\mathrm{x}}}{\partial y^{2}} \frac{\partial w_{0}}{\partial x}\right)-c_{1} E_{16}\left(\frac{\partial^{2} \phi_{\mathrm{x}}}{\partial x \partial y} \frac{\partial w_{0}}{\partial x}+\frac{\partial^{3} w_{0}}{\partial x^{2} \partial y} \frac{\partial w_{0}}{\partial x}\right) \\
& -c_{1} E_{26}\left(\frac{\partial^{2} \phi_{\mathrm{y}}}{\partial y^{2}} \frac{\partial w_{0}}{\partial x}+\frac{\partial^{3} w_{0}}{\partial y^{3}} \frac{\partial w_{0}}{\partial x}\right)-c_{1} E_{66}\left(\frac{\partial^{2} \phi_{\mathrm{y}}}{\partial x \partial y} \frac{\partial w_{0}}{\partial x}+\frac{\partial^{2} \phi_{\mathrm{x}}}{\partial y^{2}} \frac{\partial w_{0}}{\partial x}+2 \frac{\partial^{3} w_{0}}{\partial x \partial y^{2}} \frac{\partial w_{0}}{\partial x}\right) \\
& +A_{16}\left(\frac{1}{2}\left(\frac{\partial^{2} w_{0}}{\partial x^{2}}\right)^{2}+\frac{\partial u_{0}}{\partial x} \frac{\partial^{2} w_{0}}{\partial x^{2}}\right)+A_{26}\left(\frac{1}{2} \frac{\partial^{2} w_{0}}{\partial x^{2}} \frac{\partial^{2} w_{0}}{\partial y^{2}}+\frac{\partial v_{0}}{\partial y} \frac{\partial^{2} w_{0}}{\partial x^{2}}\right)+A_{66}\left(\frac{\partial w_{0}}{\partial x} \frac{\partial w_{0}}{\partial y} \frac{\partial^{2} w_{0}}{\partial x^{2}}\right. \\
& \left.+\frac{\partial u_{0}}{\partial y} \frac{\partial^{2} w_{0}}{\partial x^{2}}+\frac{\partial v_{0}}{\partial x} \frac{\partial^{2} w_{0}}{\partial x^{2}}\right)+B_{16} \frac{\partial \phi_{\mathrm{x}}}{\partial x} \frac{\partial^{2} w_{0}}{\partial x^{2}}+B_{26} \frac{\partial \phi_{\mathrm{y}}}{\partial y} \frac{\partial^{2} w_{0}}{\partial x^{2}}+B_{66}\left(\frac{\partial \phi_{\mathrm{y}}}{\partial x} \frac{\partial^{2} w_{0}}{\partial x^{2}}+\frac{\partial \phi_{\mathrm{x}}}{\partial y} \frac{\partial^{2} w_{0}}{\partial x^{2}}\right) \\
& -c_{1} E_{16}\left(\frac{\partial \phi_{\mathrm{x}}}{\partial x} \frac{\partial^{2} w_{0}}{\partial x^{2}}+\left(\frac{\partial^{2} w_{0}}{\partial x^{2}}\right)^{2}\right)-c_{1} E_{26}\left(\frac{\partial \phi_{\mathrm{y}}}{\partial y} \frac{\partial^{2} w_{0}}{\partial x^{2}}+\frac{\partial^{2} w_{0}}{\partial x^{2}} \frac{\partial^{2} w_{0}}{\partial y^{2}}\right)-c_{1} E_{66}\left(\frac{\partial \phi_{\mathrm{y}}}{\partial x} \frac{\partial^{2} w_{0}}{\partial x^{2}}\right. \\
& \left.+\frac{\partial \phi_{\mathrm{x}}}{\partial y} \frac{\partial^{2} w_{0}}{\partial x^{2}}+2 \frac{\partial^{2} w_{0}}{\partial x \partial y} \frac{\partial^{2} w_{0}}{\partial x^{2}}\right)+c_{1} E_{11}\left(\frac{\partial^{2} w_{0}}{\partial x^{2}} \frac{\partial^{2} w_{0}}{\partial x^{2}}+\frac{\partial w_{0}}{\partial x} \frac{\partial^{3} w_{0}}{\partial x^{3}}+\frac{\partial^{3} u_{0}}{\partial x^{3}}\right)+c_{1} E_{12}\left(\frac{\partial^{3} w_{0}}{\partial x^{2} \partial y} \frac{\partial w_{0}}{\partial y}\right. \\
& \left.+\frac{\partial^{2} w_{0}}{\partial x \partial y} \frac{\partial^{2} w_{0}}{\partial x \partial y}+\frac{\partial^{3} v_{0}}{\partial x^{2} \partial y}\right)+c_{1} E_{16}\left(\frac{\partial w_{0}}{\partial x} \frac{\partial^{3} w_{0}}{\partial x^{2} \partial y}+\frac{\partial^{3} w_{0}}{\partial x^{3}} \frac{\partial w_{0}}{\partial y}+2 \frac{\partial^{2} w_{0}}{\partial x^{2}} \frac{\partial^{2} w_{0}}{\partial x \partial y}+\frac{\partial^{3} u_{0}}{\partial x^{2} \partial y}+\frac{\partial^{3} v_{0}}{\partial x^{3}}\right) \\
& +c_{1} F_{11} \frac{\partial^{3} \phi_{\mathrm{x}}}{\partial x^{3}}+c_{1} F_{12} \frac{\partial^{3} \phi_{\mathrm{y}}}{\partial x^{2} \partial y}+c_{1} F_{16}\left(\frac{\partial^{3} \phi_{\mathrm{y}}}{\partial x^{3}}+\frac{\partial^{3} \phi_{\mathrm{x}}}{\partial x^{2} \partial y}\right)-c_{1}^{2} H_{11}\left(\frac{\partial^{3} \phi_{\mathrm{x}}}{\partial x^{3}}+\frac{\partial^{4} w_{0}}{\partial x^{4}}\right)-c_{1}^{2} H_{12}\left(\frac{\partial^{3} \phi_{\mathrm{y}}}{\partial x^{2} \partial y}+\frac{\partial^{4} w_{0}}{\partial x^{2} \partial y^{2}}\right) \\
& -c_{1}^{2} H_{16}\left(\frac{\partial^{3} \phi_{\mathrm{y}}}{\partial x^{3}}+\frac{\partial^{3} \phi_{\mathrm{x}}}{\partial x^{2} \partial y}+2 \frac{\partial^{4} w_{0}}{\partial x^{3} \partial y}\right)-c_{1} \frac{\partial^{2} P_{\mathrm{x}}^{T}}{\partial x^{2}}+2 c_{1} E_{16}\left(\frac{\partial^{2} w_{0}}{\partial x \partial y} \frac{\partial^{2} w_{0}}{\partial x^{2}}+\frac{\partial w_{0}}{\partial x} \frac{\partial^{3} w_{0}}{\partial x^{2} \partial y}+\frac{\partial^{3} u_{0}}{\partial x^{2} \partial y}\right) \\
& +2 c_{1} E_{26}\left(\frac{\partial^{2} w_{0}}{\partial y^{2}} \frac{\partial^{2} w_{0}}{\partial x \partial y}+\frac{\partial w_{0}}{\partial y} \frac{\partial^{3} w_{0}}{\partial x \partial y^{2}}+\frac{\partial^{3} v_{0}}{\partial x \partial y^{2}}\right) \\
& +2 c_{1} E_{66}\left(\frac{\partial^{2} w_{0}}{\partial x \partial y} \frac{\partial^{2} w_{0}}{\partial x \partial y}+\frac{\partial w_{0}}{\partial x} \frac{\partial^{3} w_{0}}{\partial x \partial y^{2}}+\frac{\partial^{3} w_{0}}{\partial x^{2} \partial y} \frac{\partial w_{0}}{\partial y}+\frac{\partial^{2} w_{0}}{\partial x^{2}} \frac{\partial^{2} w_{0}}{\partial y^{2}}+\frac{\partial^{3} u_{0}}{\partial x \partial y^{2}}+\frac{\partial^{3} v_{0}}{\partial x^{2} \partial y}\right)+2 c_{1} F_{16} \frac{\partial^{3} \phi_{x}}{\partial x^{2} \partial y} \\
& +2 c_{1} F_{26} \frac{\partial^{3} \phi_{\mathrm{y}}}{\partial x \partial y^{2}}+2 c_{1} F_{66}\left(\frac{\partial^{3} \phi_{\mathrm{y}}}{\partial x^{2} \partial y}+\frac{\partial^{3} \phi_{\mathrm{x}}}{\partial x \partial y^{2}}\right)-2 c_{1}^{2} H_{16}\left(\frac{\partial^{3} \phi_{\mathrm{x}}}{\partial x^{2} \partial y}+\frac{\partial^{4} w_{0}}{\partial x^{3} \partial y}\right)-2 c_{1}^{2} H_{26}\left(\frac{\partial^{3} \phi_{\mathrm{y}}}{\partial x \partial y^{2}}+\frac{\partial^{4} w_{0}}{\partial x \partial y^{3}}\right)
\end{aligned}
$$




$$
\begin{aligned}
& -2 c_{1}^{2} H_{66}\left(\frac{\partial^{3} \phi_{\mathrm{y}}}{\partial x^{2} \partial y}+\frac{\partial^{3} \phi_{\mathrm{x}}}{\partial x \partial y^{3}}+2 \frac{\partial^{4} w_{0}}{\partial x^{2} \partial y^{2}}\right)+c_{1} E_{12}\left(\frac{\partial^{2} w_{0}}{\partial x \partial y} \frac{\partial^{2} w_{0}}{\partial x \partial y}+\frac{\partial w_{0}}{\partial x} \frac{\partial^{3} w_{0}}{\partial x \partial y^{2}}+\frac{\partial^{3} u_{0}}{\partial x \partial y^{2}}\right) \\
& +c_{1} E_{22}\left(\frac{\partial^{3} w_{0}}{\partial y^{3}} \frac{\partial w_{0}}{\partial y}+\frac{\partial^{2} w_{0}}{\partial y^{2}} \frac{\partial^{2} w_{0}}{\partial y^{2}}+\frac{\partial^{3} v_{0}}{\partial y^{3}}\right)+c_{1} E_{26}\left(\frac{\partial w_{0}}{\partial x} \frac{\partial^{3} w_{0}}{\partial y^{3}}+\frac{\partial^{3} w_{0}}{\partial x \partial y^{2}} \frac{\partial w_{0}}{\partial y}+2 \frac{\partial^{2} w_{0}}{\partial x \partial y} \frac{\partial^{2} w_{0}}{\partial y^{2}}\right. \\
& \left.+\frac{\partial^{3} u_{0}}{\partial y^{3}}+\frac{\partial^{3} v_{0}}{\partial x \partial y^{2}}\right)+c_{1} F_{12} \frac{\partial^{3} \phi_{\mathrm{x}}}{\partial x \partial y^{2}}+c_{1} F_{22} \frac{\partial^{3} \phi_{\mathrm{y}}}{\partial y^{3}}+c_{1} F_{26}\left(\frac{\partial^{3} \phi_{\mathrm{y}}}{\partial x \partial y^{2}}+\frac{\partial^{3} \phi_{\mathrm{x}}}{\partial y^{3}}\right)-c_{1}^{2} H_{12}\left(\frac{\partial^{3} \phi_{\mathrm{x}}}{\partial x \partial y^{2}}+\frac{\partial^{4} w_{0}}{\partial x^{2} \partial y^{2}}\right) \\
& -c_{1}^{2} H_{22}\left(\frac{\partial^{3} \phi_{\mathrm{y}}}{\partial y^{3}}+\frac{\partial^{4} w_{0}}{\partial y^{4}}\right)-c_{1}^{2} H_{26}\left(\frac{\partial^{3} \phi_{\mathrm{y}}}{\partial x \partial y^{2}}+\frac{\partial^{3} \phi_{\mathrm{x}}}{\partial y^{3}}+2 \frac{\partial^{4} w_{0}}{\partial x \partial y^{3}}\right)-c_{1} \frac{\partial^{2} P_{\mathrm{y}}^{T}}{\partial y^{2}}+q+P_{\mathrm{a}}-\mu \dot{w}_{0} \\
& =I_{0} \ddot{w}_{0}+I_{3} c_{1} \frac{\partial \ddot{u}_{0}}{\partial x}+I_{3} c_{1} \frac{\partial \ddot{v}_{0}}{\partial x}+I_{4} c_{1} \frac{\partial \ddot{\phi}_{\mathrm{x}}}{\partial x}+I_{4} c_{1} \frac{\partial \ddot{\phi}_{\mathrm{y}}}{\partial y}-I_{6} c_{1}^{2} \frac{\partial \ddot{\phi}_{\mathrm{x}}}{\partial x}-I_{6} c_{1}^{2} \frac{\partial \ddot{\phi}_{\mathrm{y}}}{\partial y}-I_{6} c_{1}^{2} \frac{\partial^{2} \ddot{w}_{0}}{\partial x^{2}} \\
& -I_{6} c_{1}^{2} \frac{\partial^{2} \ddot{w}_{0}}{\partial y^{2}}
\end{aligned}
$$

The boundary conditions for the simply supported plate are

$$
\begin{array}{ll}
v=w=w_{0}=N_{\mathrm{x}}=M_{\mathrm{x}}=\partial^{2} w_{0} / \partial x^{2}=0, & \text { at } x=0, a \\
u=w=w_{0}=N_{\mathrm{y}}=M_{\mathrm{y}}=\partial^{2} w_{0} / \partial y^{2}=0, & \text { at } y=0, b
\end{array}
$$

The modal expansions for the generalized displacements $u_{0}, v_{0}, w_{0}, \phi_{\mathrm{x}}$, and $\phi_{\mathrm{y}}$ are described as

$$
\begin{aligned}
& u_{0}=\sum_{\mathrm{i}=1}^{\mathrm{M}} \sum_{\mathrm{j}=1}^{\mathrm{N}} u_{\mathrm{ij}}(t) \cos \left(\frac{i \pi x}{a}\right) \sin \left(\frac{j \pi y}{b}\right) \\
& v_{0}=\sum_{\mathrm{i}=1}^{\mathrm{M}} \sum_{\mathrm{j}=1}^{\mathrm{N}} v_{\mathrm{ij}}(t) \sin \left(\frac{i \pi x}{a}\right) \cos \left(\frac{j \pi y}{b}\right) \\
& w_{0}=\sum_{\mathrm{i}=1}^{\mathrm{M}} \sum_{\mathrm{j}=1}^{\mathrm{N}} w_{\mathrm{ij}}(t) \sin \left(\frac{i \pi x}{a}\right) \sin \left(\frac{j \pi y}{b}\right) \\
& \phi_{\mathrm{x}}=\sum_{\mathrm{i}=1}^{\mathrm{M}} \sum_{\mathrm{j}=1}^{\mathrm{N}} \phi_{\mathrm{ij}}(t) \cos \left(\frac{i \pi x}{a}\right) \sin \left(\frac{j \pi y}{b}\right) \\
& \phi_{\mathrm{y}}=\sum_{\mathrm{i}=1}^{\mathrm{M}} \sum_{\mathrm{j}=1}^{\mathrm{N}} \phi_{\mathrm{ij}}(t) \sin \left(\frac{i \pi x}{a}\right) \cos \left(\frac{j \pi y}{b}\right)
\end{aligned}
$$

The transverse excitation is described as 


$$
F=\sum_{\mathrm{i}=1}^{\mathrm{M}} \sum_{\mathrm{j}=1}^{\mathrm{N}} F_{\mathrm{ij}}(t) \sin \left(\frac{i \pi x}{a}\right) \sin \left(\frac{j \pi y}{b}\right)
$$

where $F_{\mathrm{ij}}$ denotes the amplitude of the transverse excitation corresponding to the modes of transverse deflection. By substituting Eqs. (22-23) into Eqs. (21a)-(21e) and applying the Galerkin procedure to discretize the equations, the four coupled algebraic equations are obtained, and the expressions of $u, v, \phi_{\mathrm{x}}$, and $\phi_{\mathrm{y}}$ only contain $w$. The nonlinear ordinary differential equation containing only $\boldsymbol{W}$ is obtained as

$$
\ddot{W}+\mu \dot{W}+M W+N L=F \cos \Omega t
$$

where $\boldsymbol{W}=\left[\mathrm{W}_{1}, \mathrm{~W}_{2}\right]^{\mathrm{T}}$ is the transverse displacements vectors, $\boldsymbol{\mu}$ is the aerodynamic damping, $\boldsymbol{M}$ is the mass matrix, $\boldsymbol{N} \boldsymbol{L}$ is the nonlinear stiffness vector, and $\boldsymbol{F}$ is the amplitude excitation matrix. Based on Eq. (24), the time histories and phase portraits of PMMA/CNT composite plate are obtained to analyze the dynamic characteristics.

\section{Results and discussion}

The molecular simulation results of SWCNT, PMMA polymer, and PMMA/SWCNT nanocomposite in various temperature and pressure conditions are presented herein, which are used in the multiscale simulation of FG-based composite plates, and the obtained nonlinear dynamic responses are discussed.

\subsection{Nano-mechanical analysis}

During the last $1.0 \mathrm{~ns}$ NPT relaxation process, the densities of PMMA polymer and PMMA/SWCNT nanocomposites are recorded, and the data from the last $50 \mathrm{ps}$ is averaged as shown in Table 2. The density of PMMA polymer in Case \#1 is calculated as $1.184 \pm 0.001 \mathrm{~g} \cdot \mathrm{cm}^{-3}$, which is close to the experiment result of $1.190 \mathrm{~g} \cdot \mathrm{cm}^{-3}$ and the simulation result of $1.180 \mathrm{~g} \cdot \mathrm{cm}^{-3}$ at room temperature $[39,57]$. With the decrease of temperature and pressure, the density shows a slight increase, with the value of 1.190 $\pm 0.002,1.193 \pm 0.001$, and $1.195 \pm 0.002 \mathrm{~g} \cdot \mathrm{cm}^{-3}$ in Case \#2, \#3, and \#4, respectively. The observed increasing trend of density agrees very well with the experimental measurement of other polymer, where the density continues to increase as the 
temperature decreases from 343.15 to $283.15 \mathrm{~K}$, and the pressure decreases from 35 to 0.1 MPa [58]. With the addition of 1 vol\% SWCNT, the density of PMMA/SWCNT nanocomposites shows a consistent increase compared with pure PMMA polymer in all the investigated cases, with the value of $1.202,1.206,1.210$, and $1.214 \mathrm{~g} \cdot \mathrm{cm}^{-3}$ in Case $\# 1, \# 2, \# 3$ and \#4 respectively, as shown in Table 2 . The increment of about $1.5 \%$ due to the added 1 vol\% SWCNT has been observed in previous experimental and simulation studies of other polymer/CNT nanocomposites at the temperature of $300 \mathrm{~K}$ and pressure of $1 \mathrm{~atm}$, with the increases of $1.4 \%$ in experiment and of $1.9 \%$ in MD simulation $[59,60]$. It is shown that a closely packed PMMA structure can be formed in the vicinity of the PMMA-SWCNT interface, which could contribute to the increase of the PMMA/SWCNT nanocomposite density [25, 59].

Table 2 Mechanical properties of SWCNT, PMMA, and PMMA/SWCNT nanocomposite.

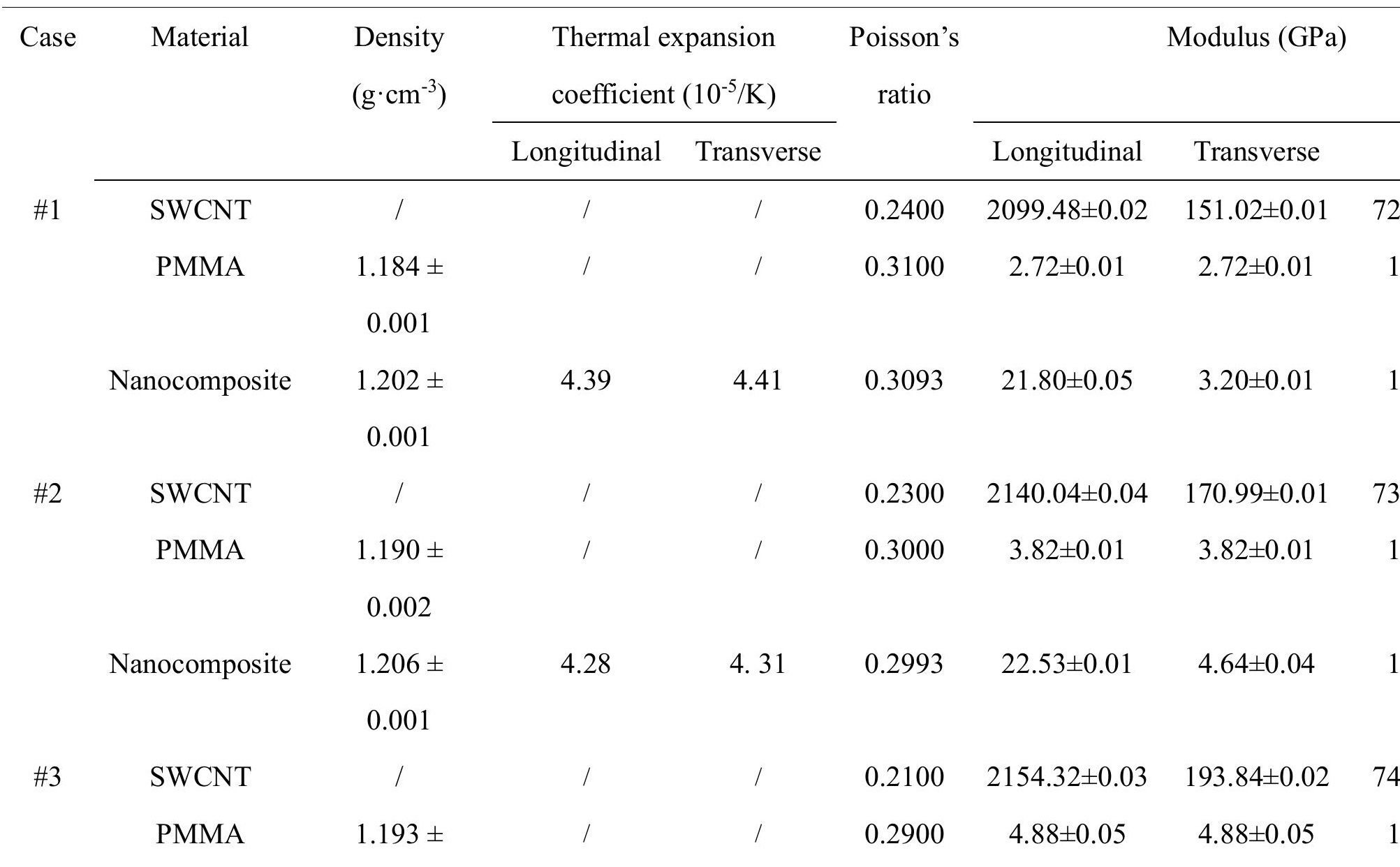




\begin{tabular}{|c|c|c|c|c|c|c|}
\hline Nanocomposite & $\begin{array}{c}1.210 \pm \\
0.001\end{array}$ & 4.09 & 4.17 & 0.2892 & $23.40 \pm 0.08$ & $5.40 \pm 0.01$ \\
\hline SWCNT & I & I & I & 0.1900 & $2158.32 \pm 0.07$ & $210.83 \pm 0.04$ \\
\hline PMMA & $\begin{array}{c}1.195 \pm \\
0.002\end{array}$ & / & / & 0.2800 & $4.96 \pm 0.03$ & $4.96 \pm 0.03$ \\
\hline Nanocomposite & $\begin{array}{c}1.214 \pm \\
0.001\end{array}$ & 3.69 & 3.89 & 0.2791 & $24.84 \pm 0.02$ & $6.74 \pm 0.03$ \\
\hline
\end{tabular}

After characterizing the density, the TEC of PMMA/SWCNT nanocomposites in the various temperature and pressure conditions are evaluated. Accordingly, the longitudinal and transverse lengths of simulation cell are recorded, as shown in Figs. 4(a-d) corresponding to Cases \#1-\#4 respectively. It is observed that the length of PMMA/SWCNT nanocomposite model increases linearly with the increased temperature. The longitudinal and transverse TEC of PMMA/SWCNT nanocomposite are calculated according to Eq. (5), as shown in Table 2. In Case \#1, the longitudinal and transverse TEC are calculated as $4.39 \times 10^{-5}$ and $4.41 \times 10^{-5} / \mathrm{K}$, which are close to the calculated results of the Schapery model based on the molecular simulation results of $4.46 \times 10^{-5}$ and $4.47 \times 10^{-5} / \mathrm{K}$, respectively [61]. With the decreased temperature and pressure in Case \#2 to \#4, the TEC decreases continuously, with the values of $4.28 \times 10^{-}$ $5,4.09 \times 10^{-5}$, and $3.69 \times 10^{-5} / \mathrm{K}$ for longitudinal direction, and $4.31 \times 10^{-5}, 4.17 \times 10^{-5}$, and $3.89 \times 10^{-5} / \mathrm{K}$ for transverse direction, respectively. It is probably because that the decreased temperature leads to the shrinkage of simulation box and the decreased deformation of molecular cell [62]. The decrease trend of TEC with the decrease of temperature for PMMA/SWCNT nanocomposite agrees very well with other polymer/CNT nanocomposite [63-65]. It is noted that the TEC in the longitudinal direction is smaller than that in the transverse direction, as the SWCNT restricts the expansion of matrix in the longitudinal direction, and enforces the matrix expansion in the transverse direction [66]. 

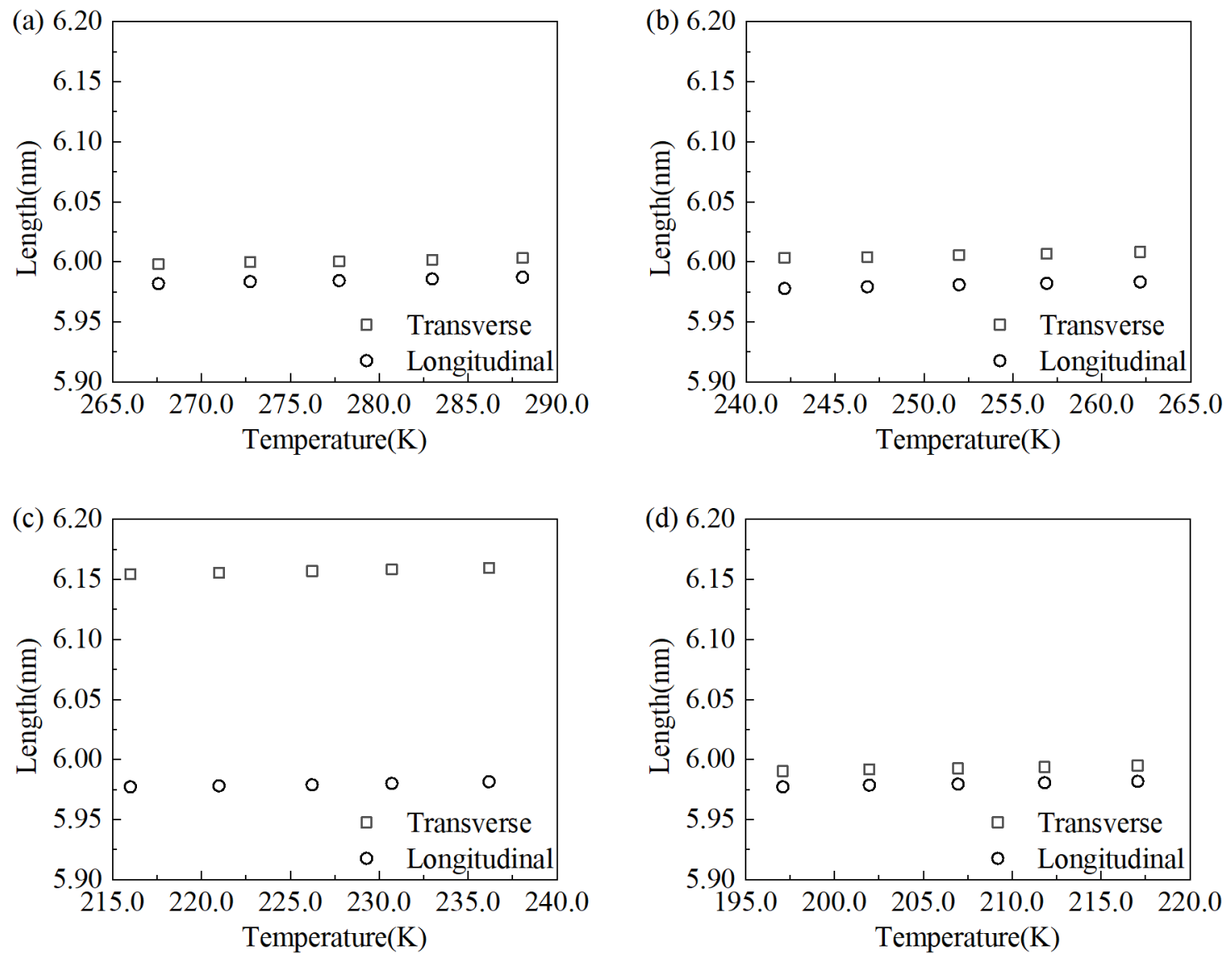

Fig.4 The longitudinal and transverse length of simulation cell in PMMA/SWCNT nanocomposite as a function of temperature: Case (a)\#1, (b) \#2, (c) \#3, and (d) \#4.

After determining the density and TEC, the Poisson's ratio of SWCNT, PMMA polymer, and PMMA/SWCNT nanocomposite in the various temperature and pressure conditions are calculated, as listed in Table 2. In Case \#1, Poisson's ratio of SWCNT, PMMA polymer, and PMMA/SWCNT nanocomposite are calculated as 0.2400 , 0.3100 , and 0.3093 , respectively, which are close to the corresponding experimental and simulation results of $0.25,0.33$, and 0.33 [67-69]. It is seen that with the decrease of temperature and pressure in the investigated cases, Poisson's ratio of SWCNT, PMMA polymer and PMMA/SWCNT nanocomposite show a monotonous decrease, which agrees with finite element simulation, MD simulation and rule of mixtures [61, $68,70]$, where the values show a similar decreased trend with the decreased 
temperature. It is probably because that the transverse moduli of SWCNT, PMMA polymer, and PMMA/SWCNT nanocomposite increase with the decrease of temperature and pressure. As the deformation process is determined by the thermal energy and strain energy, the thermal energy is reduced with the decreased temperature, and thus the strain energy required for the deformation increased [62], which leads to the decreased transverse deformation during the longitudinal tensile deformation, causing the decrease of Poisson's ratio [71].

Apart from the density, TEC, and Poisson's ratio, the moduli of SWCNT, PMMA polymer, and PMMA/SWCNT nanocomposite are quantified using the MD deformation. The stress-strain curves of SWCNT, PMMA polymer, and PMMA/SWCNT nanocomposite are shown in Fig. 5, where L, T, and S denote the longitudinal, transverse, and shear deformation respectively. From the stress-strain curves depicted in Fig. 5(a), it is shown that the longitudinal stress of SWCNT is higher than those along the transverse and shear directions. In Case \#1, the longitudinal, transverse, and shear modulus of SWCNT are estimated as 2099.48 $\pm 0.02,151.02 \pm 0.01$, and $720.25 \pm 0.06 \mathrm{GPa}$, respectively, as shown in Table 2. The longitudinal and transverse moduli are close to the simulation results of 2000 and $142 \mathrm{GPa}$, respectively, and the shear modulus is in the range of 550-860 GPa [72-74]. With the decrease of temperature and pressure in other cases, there is no obvious variation of the longitudinal, transverse, and shear stresses. 

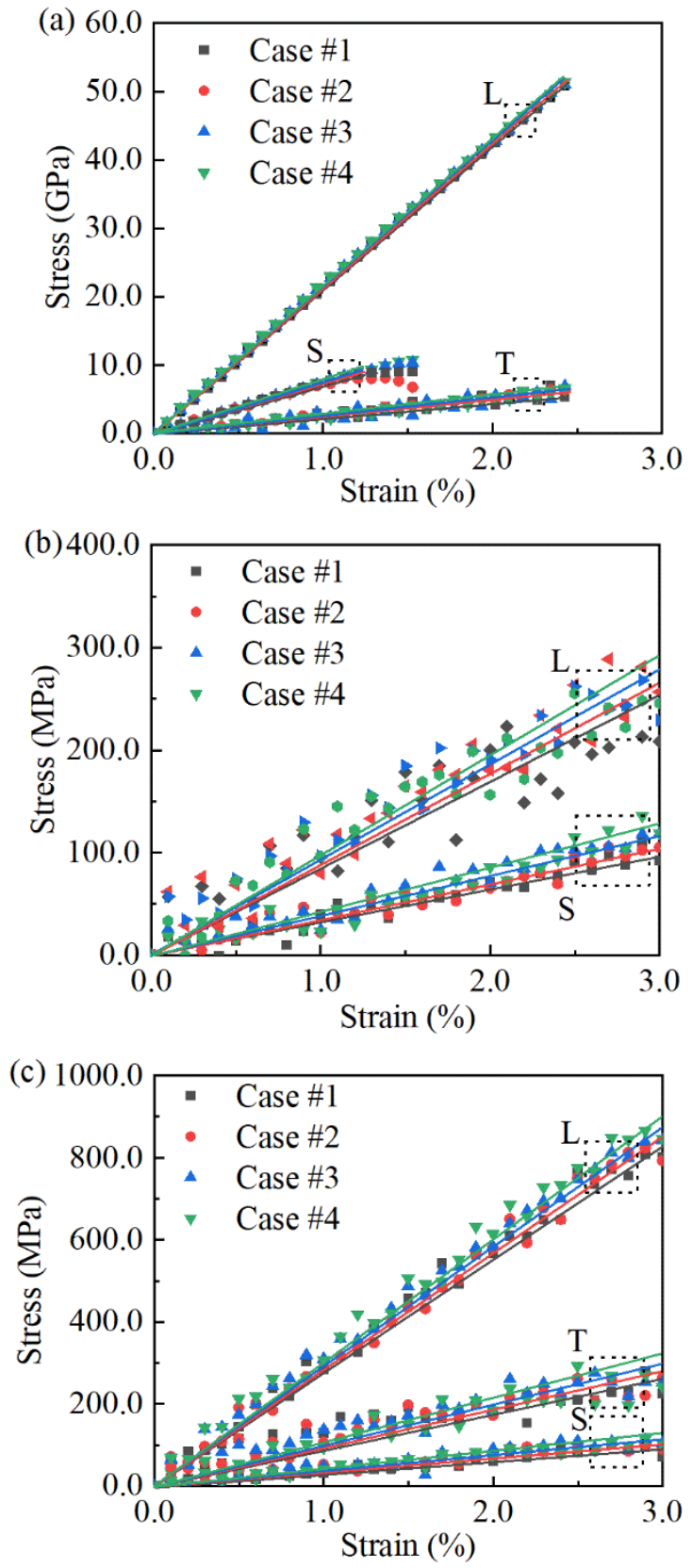

Fig. 5 Stress-strain curves of (a) SWCNT, (b) PMMA, and (c) PMMA/SWCNT nanocomposite, where $\mathrm{L}, \mathrm{T}$, and $\mathrm{S}$ denote the longitudinal, transverse, and shear deformation.

For PMMA polymer, it is observed from Fig. 5(b) that the longitudinal and shear stress level increases gradually with the decreased temperature and pressure in the investigated cases, and the longitudinal stress is higher than the transverse result. The longitudinal, transverse, and shear moduli of PMMA polymer are summarized in Table 
2. In Case \#1, it is evaluated that the polymer has a longitudinal modulus of $2.72 \pm 0.01$ $\mathrm{GPa}$, which is in the range between 2.24 and 3.80 GPa of experiments [25]. The shear modulus of the polymer is estimated as $1.04 \pm 0.04 \mathrm{GPa}$, which is close to MD simulation result of $1.19 \mathrm{GPa}[68]$. With the decrease of temperature and pressure, the longitudinal modulus increase continuously, with the value of $3.82 \pm 0.01,4.88 \pm 0.05$, and $4.96 \pm 0.03$ Gpa in Case \#2, \#3, and \#4, respectively. The increasing trend is consistent with the MD results of 3.85, 4.92, and 5.01 GPa obtained at the temperature level of 262.15, 236.15, and $216.65 \mathrm{~K}$, respectively [75].

With the addition of 1.0 vol\% SWCNT, the nanocomposite show the increased longitudinal stress level compared with PMMA polymer case, while no obvious increase is observed for the transverse and shear deformations, as shown in Fig. 5(c). In Case \#1, the longitudinal, transverse, and shear modulus of nanocomposite is calculated as $21.80 \pm 0.05,3.20 \pm 0.01$, and $1.60 \pm 0.02 \mathrm{GPa}$, which are close to the simulation results of $22.30,3.45$, and $1.62 \mathrm{GPa}$, respectively [50, 76]. Compared with the PMMA polymer, the increase of longitudinal modulus of nanocomposite is because that the SWCNT carries most of the load in the longitudinal deformation, and the high longitudinal modulus of SWCNT results in a significant increase. Comparatively, the effects of SWCNT on the transverse and shear moduli are not obvious, as that SWCNT has a comparatively low transverse and shear modulus than the longitudinal one. With the decrease of temperature and pressure, the longitudinal, transverse, and shear moduli of PMMA/SWCNT nanocomposite show similar increase as the PMMA polymer case, with the value of $22.53 \pm 0.01,23.40 \pm 0.08$, and $24.84 \pm 0.02$ Gpa in longitudinal direction. The increasing trend is consistent with the results obtained from the extended rule of mixtures for PMMA/SWCNT nanocomposite at the decreased temperature level [61].

\subsection{Nonlinear dynamic analysis}

The efficiency parameters of PMMA/SWCNT composite are derived through matching the longitudinal, transverse, and shear moduli calculated from the EROM with those from MD simulations. The results with various CNT volume fractions are 
shown in Table 3. Based on the obtained efficiency parameters, the longitudinal, transverse, and shear moduli of UD and FG-X plates are calculated using Eqs. (1-3). The input parameters $\rho_{\mathrm{a}}$ and $V_{\mathrm{a}}$ of the quasi-steady first-order piston theory are calculated via Eqs. (7-8) and listed in Table 3. The Mach number used in this study is determined as 1.5 [77].

Table 3 Material parameters in different temperature and pressure conditions.

\begin{tabular}{|c|c|c|c|c|c|c|c|c|c|c|c|}
\hline \multirow[t]{3}{*}{ Case } & \multicolumn{3}{|c|}{ Efficiency parameters } & \multicolumn{6}{|c|}{ Modulus (GPa) } & \multirow{3}{*}{$\begin{array}{c}\rho_{\mathrm{a}} \\
\left(\mathrm{kg} \cdot \mathrm{m}^{-}\right. \\
\left.{ }^{-}\right)\end{array}$} & \multirow{3}{*}{$\begin{array}{c}V_{\mathrm{a}}(\mathrm{m} \cdot \mathrm{s} \\
\left.{ }^{1}\right)\end{array}$} \\
\hline & \multirow[t]{2}{*}{$\eta_{\mathrm{x}}$} & \multirow[t]{2}{*}{$\eta_{\mathrm{y}}$} & \multirow[t]{2}{*}{$\eta_{\mathrm{xy}}$} & \multicolumn{2}{|c|}{ Longitudinal } & \multicolumn{2}{|c|}{ Transverse } & \multicolumn{2}{|c|}{ Shear } & & \\
\hline & & & & UD & FG-X & UD & FG-X & UD & FG-X & & \\
\hline$\# 1$ & 0.9101 & 1.1650 & 1.5231 & 21.80 & 33.25 & 3.20 & 3.22 & 1.60 & 1.61 & 1.225 & 0 \\
\hline$\# 2$ & 0.8761 & 1.2028 & 1.2594 & 22.53 & 33.76 & 4.64 & 4.67 & 1.87 & 1.88 & 0.819 & 136.0516 \\
\hline$\# 3$ & 0.8619 & 1.0958 & 1.2729 & 23.40 & 34.51 & 5.40 & 5.43 & 2.43 & 2.44 & 0.526 & 305.2914 \\
\hline$\# 4$ & 0.9234 & 1.3456 & 1.5003 & 24.84 & 36.77 & 6.74 & 6.78 & 2.94 & 2.96 & 0.312 & 547.8169 \\
\hline
\end{tabular}

The phase planes and time histories of PMMA/CNT composite plate under nonlinear forced vibration for Case \#1-4 are depicted in Figs. 6-9, respectively. For different CNT distribution types, the periodic motions for Case \#2-4 have been detected under the same external excitation and damping condition, as shown in Figs. 7-9. In addition, the quasi-periodic motion and chaotic motion for Case \#1 are demonstrated in Figs. 6(e-h) and Figs. 6(a-d), respectively. For the results of the two CNT distributions in each investigated case, the upper row is the first-order vibration, and lower row is the second-order vibration. It is clearly shown that the amplitude of first-order vibration is significantly larger than the second-order vibration, and such trend is consistent with Ref. [78]. Meanwhile, the maximum amplitude of nonlinear forced vibration for FG-X type is smaller than that of UD distribution, which is due to the fact that FG-X types of CNT distribution effectively enhances the stiffness of plates, as demonstrated in Table 3 , where the longitudinal, transverse and shear moduli of the composite plate with the FG-X distribution are improved significantly compared with the UD distribution. In the 
other investigated cases, it is observed that with the decreased temperature and pressure, the amplitude of PMMA/CNT composite plate with UD and FG-X types both decrease, as shown in Figs. 7-9. This is because that the longitudinal, transverse, and shear moduli of PMMA/SWCNT composite plate increase gradually with the decrease of temperature and pressure levels, as described in Section 4.1, which causes the increase of stiffness and leads to the decrease of the vibration amplitude. 

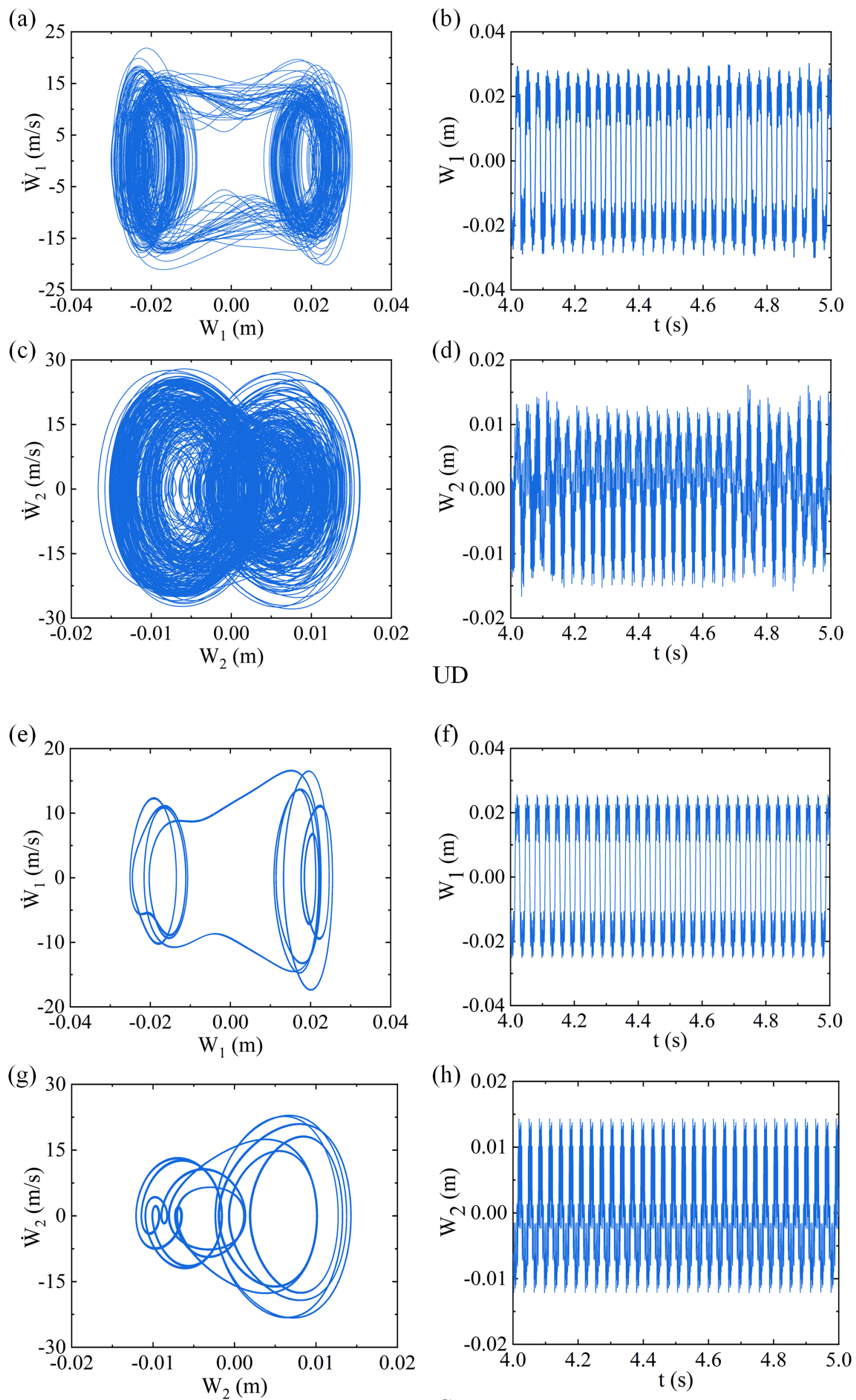

FG-X 
Fig. 6 Phase planes and time histories of PMMA/CNT composite plate under nonlinear forced vibration for Case \#1: the (a, e) phase portraits on planes $\left(\mathrm{W}_{1}, \dot{\mathrm{W}}_{1}\right),(\mathrm{b}, \mathrm{f})$ time history diagrams on planes $\left(\mathrm{t}, \mathrm{W}_{1}\right),(\mathrm{c}, \mathrm{g})$ phase portraits on planes $\left(\mathrm{W}_{2}, \dot{\mathrm{W}}_{2}\right),(\mathrm{d}, \mathrm{h})$ time history diagrams on planes $\left(\mathrm{t}, \mathrm{W}_{2}\right)$. 

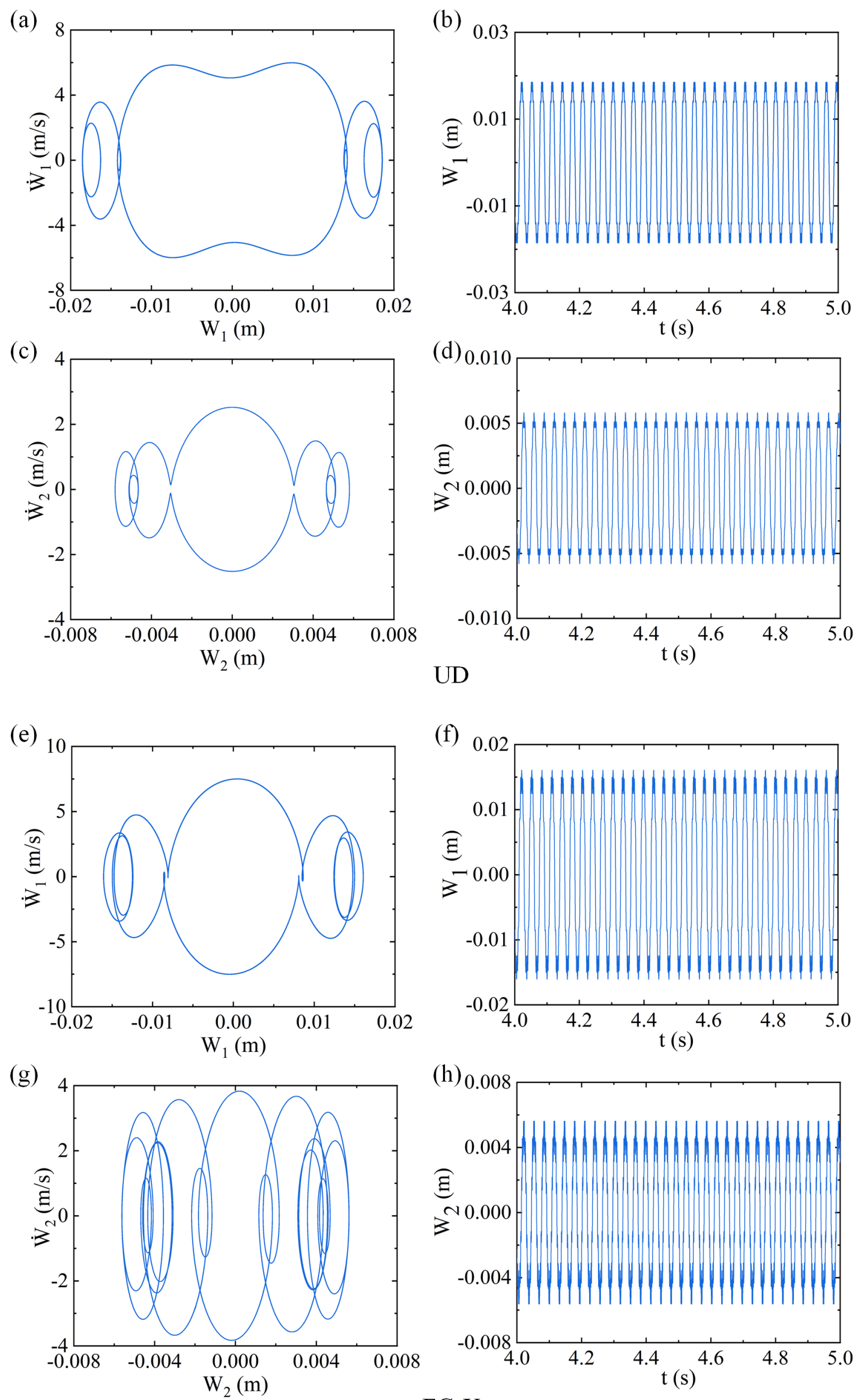

FG-X 
Fig. 7 Phase planes and time histories of PMMA/CNT composite plate under nonlinear forced vibration for Case \#2: the (a, e) phase portraits on planes $\left(\mathrm{W}_{1}, \dot{\mathrm{W}}_{1}\right),(\mathrm{b}, \mathrm{f})$ time history diagrams on planes $\left(\mathrm{t}, \mathrm{W}_{1}\right),(\mathrm{c}, \mathrm{g})$ phase portraits on planes $\left(\mathrm{W}_{2}, \dot{\mathrm{W}}_{2}\right),(\mathrm{d}, \mathrm{h})$ time history diagrams on planes $\left(\mathrm{t}, \mathrm{W}_{2}\right)$. 
(a)

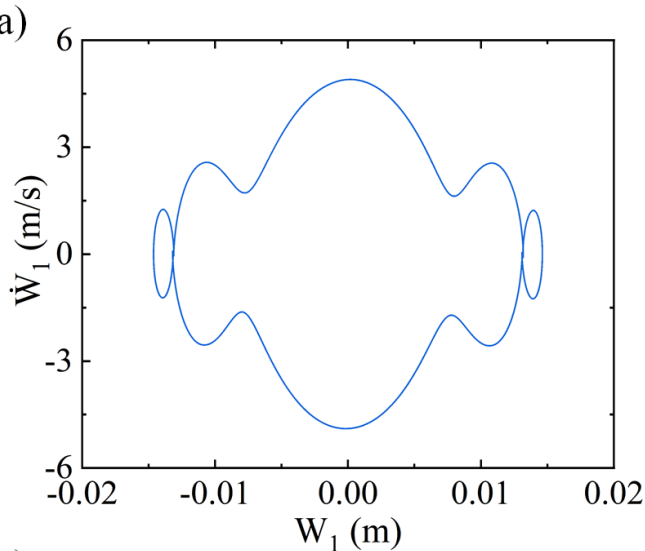

(c)

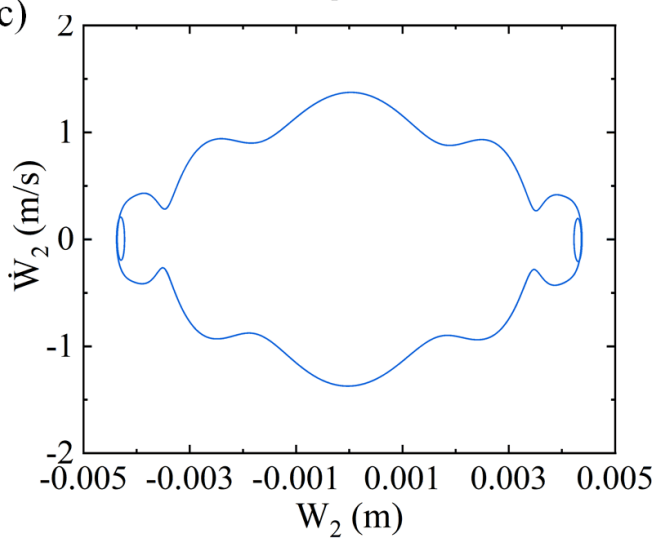

(e)

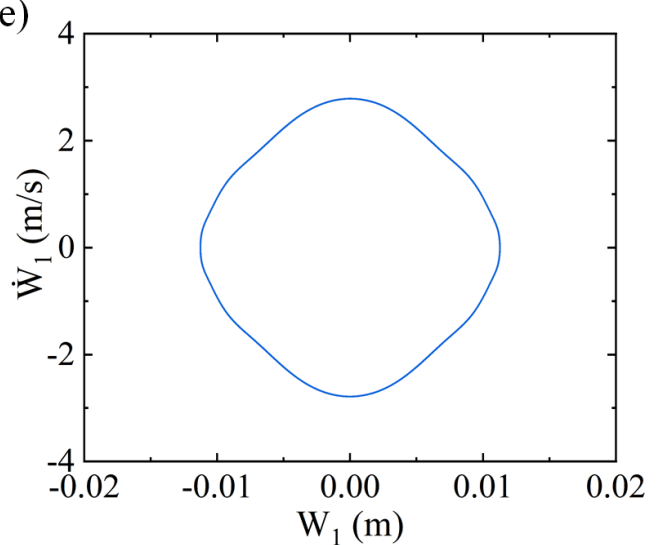

(g)

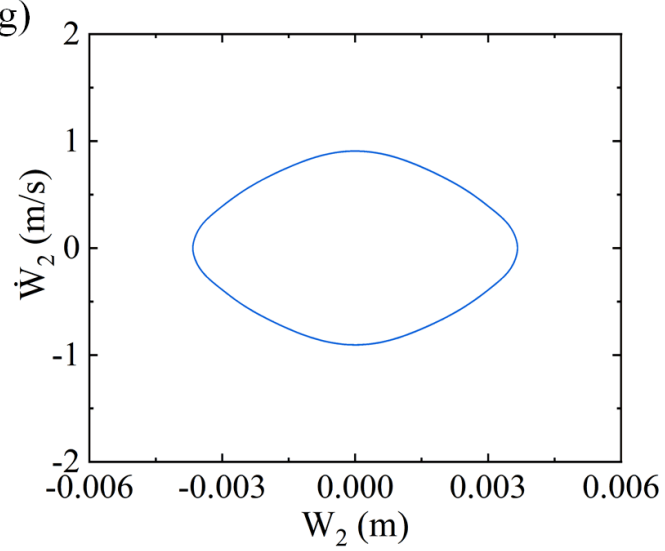

(b)

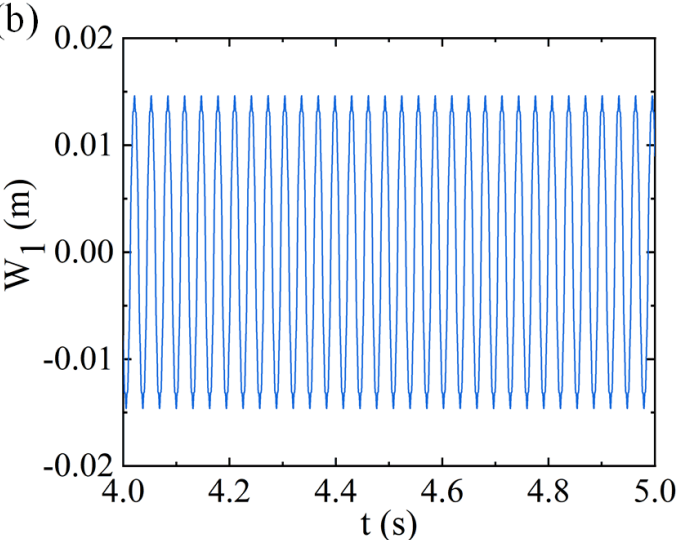

(d) 0.00

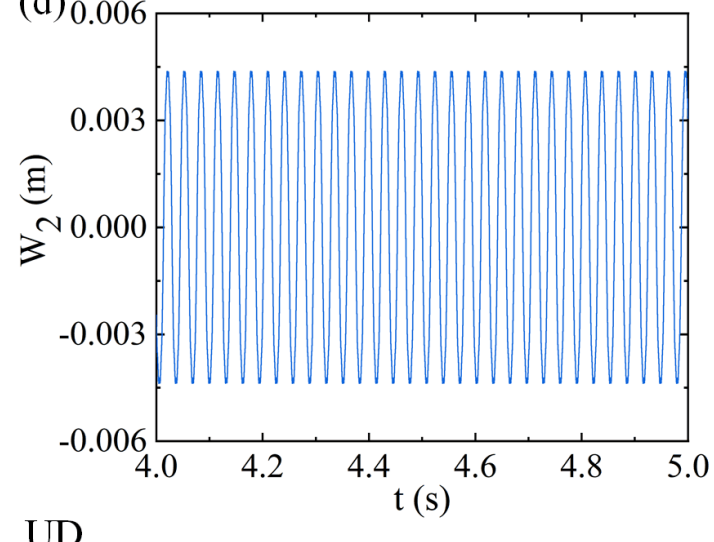

UD
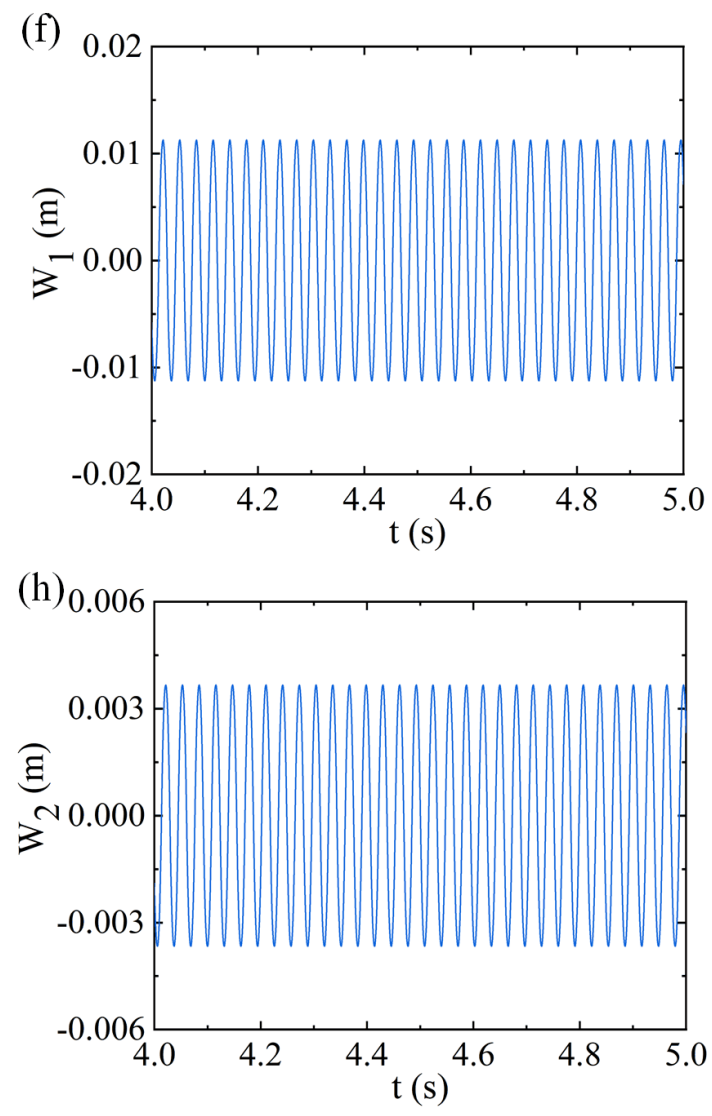

FG-X 
Fig. 8 Phase planes and time histories of PMMA/CNT composite plate under nonlinear forced vibration for Case \#3: the (a, e) phase portraits on planes $\left(\mathrm{W}_{1}, \dot{\mathrm{W}}_{1}\right),(\mathrm{b}, \mathrm{f})$ time history diagrams on planes $\left(\mathrm{t}, \mathrm{W}_{1}\right),(\mathrm{c}, \mathrm{g})$ phase portraits on planes $\left(\mathrm{W}_{2}, \dot{\mathrm{W}}_{2}\right),(\mathrm{d}, \mathrm{h})$ time history diagrams on planes $\left(\mathrm{t}, \mathrm{W}_{2}\right)$. 
(a)

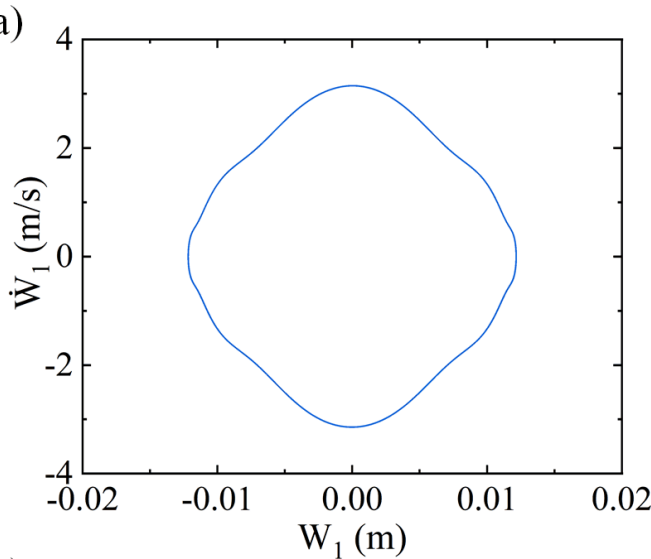

(c)

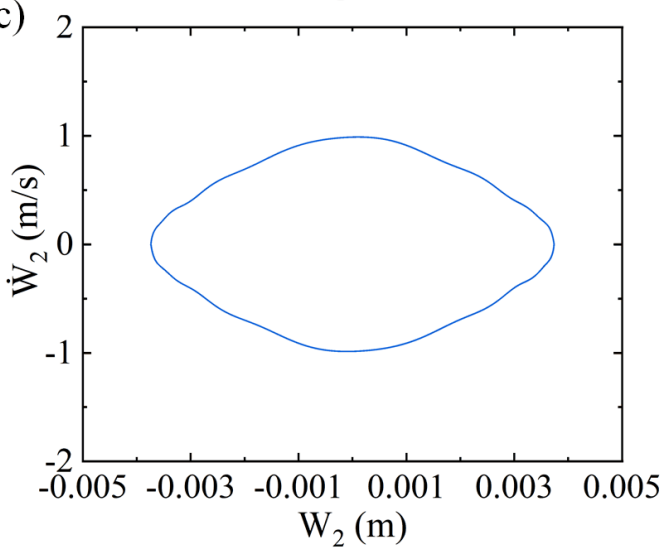

(b)
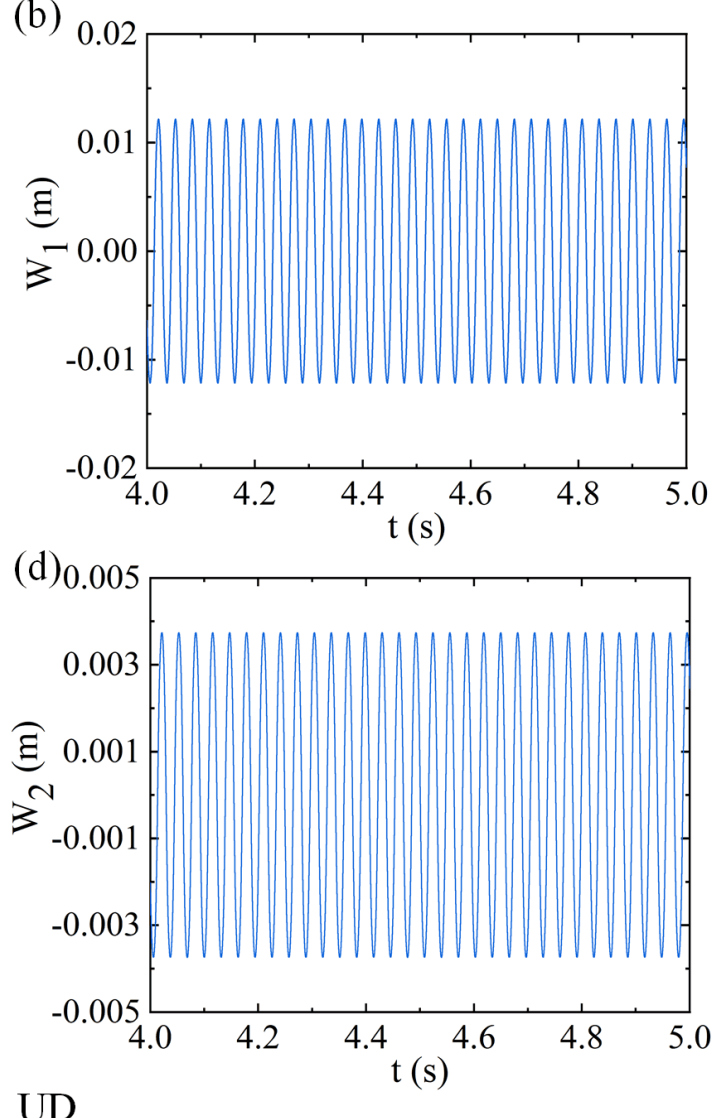

(f)
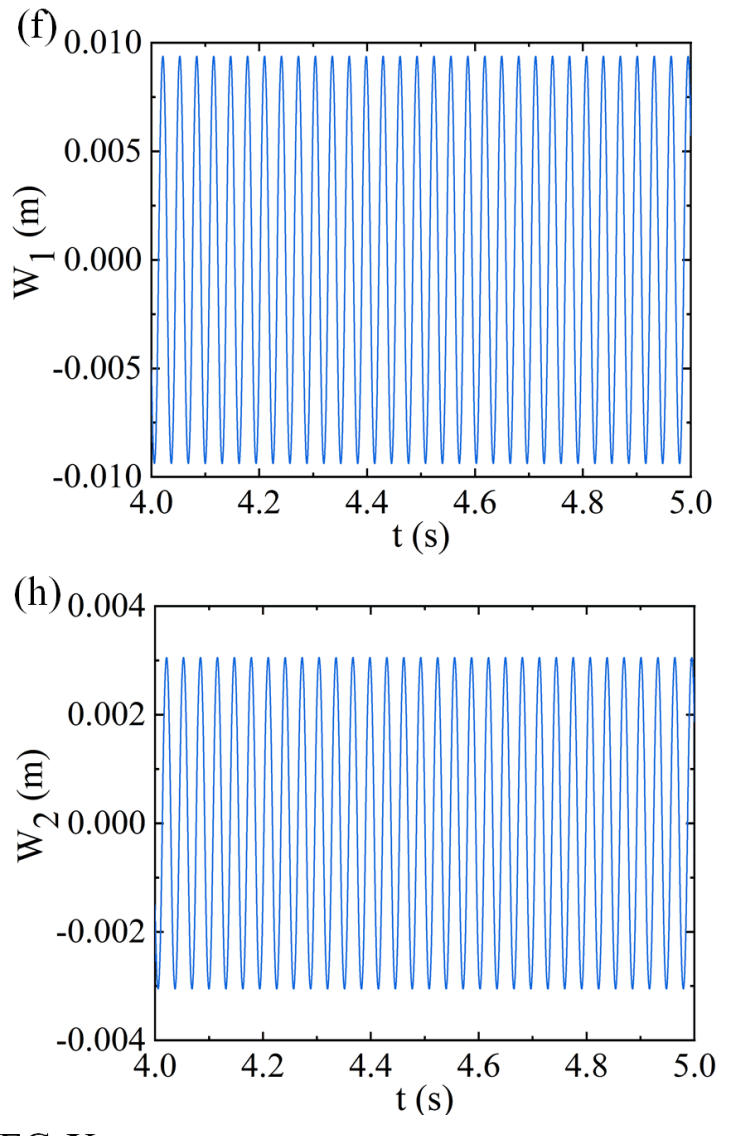

FG-X (e)

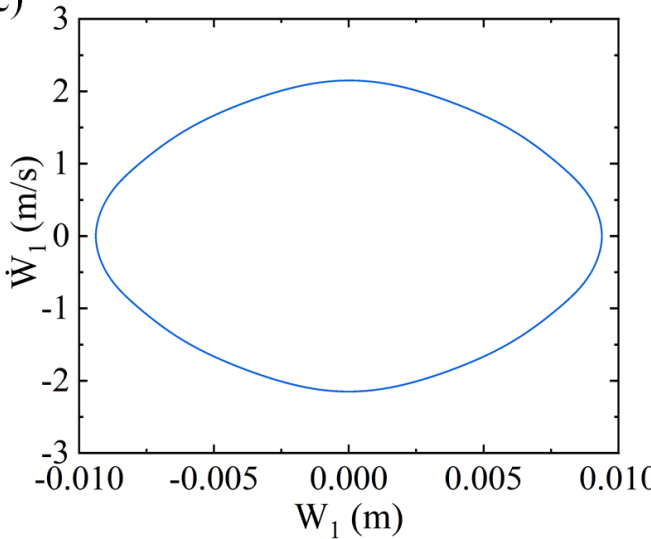

(g)

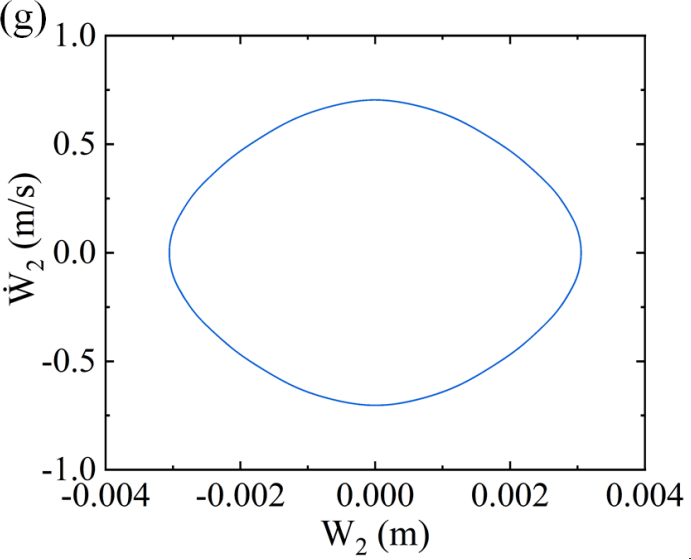


Fig. 9 Phase planes and time histories of PMMA/CNT composite plate under nonlinear forced vibration for Case \#4: the (a, e) phase portraits on planes $\left(\mathrm{W}_{1}, \dot{\mathrm{W}}_{1}\right),(\mathrm{b}, \mathrm{f})$ time history diagrams on planes $\left(\mathrm{t}, \mathrm{W}_{1}\right),(\mathrm{c}, \mathrm{g})$ phase portraits on planes $\left(\mathrm{W}_{2}, \dot{\mathrm{W}}_{2}\right),(\mathrm{d}, \mathrm{h})$ time history diagrams on planes $\left(\mathrm{t}, \mathrm{W}_{2}\right)$.

\section{Conclusions}

In this study, the nonlinear dynamic response of PMMA/CNT composite plate in the different temperature and pressure conditions is investigated using a MD-based multiscale approach. Firstly, MD simulation is applied to calculate the longitudinal, transverse, shear modulus, the TEC, and Poisson's ratio of SWCNT, PMMA polymer, and PMMA/SWCNT nanocomposite in the coupled temperature and pressure conditions. Secondly, PMMA/SWCNT nanocomposites are utilized as microinclusions in a micro-mechanical approach to estimate the macroscopic response of composite plate. Finally, the nonlinear dynamic response of composite plate is analyzed using macroscale simulation. It is shown that the amplitude of PMMA/CNT composite plate decreases with the decrease of temperature and pressure conditions under the constant load conditions. Meanwhile, the second-order maximum vibration amplitude is significantly lower than the first-order. The nonlinear dynamic response of PMMA/CNT composite plate is studied, which contributes to the understanding of the long-term dynamic behavior of composite materials during the service-life in the aerospace field. Furthermore, the quantitative transfer of physical and mechanical properties between molecular and macroscale scales is established to provide a theoretical basis for the design and optimization of FG-CNTRC.

\section{Acknowledgments}

The authors gratefully acknowledge the support of National Natural Science Foundation of China through Grants No. 12072003 and 51808020.

Data Availability The datasets generated during and/or analysed during the current study are available from the corresponding author on reasonable request. 


\section{Declarations}

Conflicts of interest The authors declare that they have no conflict of interest.

\section{References}

1. Haohan, Q., Yuanming, D., Jia, C.: Research in thermo-physical property testing system of aeronautic PMMA based on temperature-pressure parameters. Adv. Mater. Res. 647, 828-832 (2013).

2. Hu, W., Guo, H., Chen, Y., Xie, R., Jing, H., He, P.: Experimental investigation and modeling of the rate-dependent deformation behavior of PMMA at different temperatures. Eur. Polym. J. 85, 313-323 (2016).

3. Mwafy, E.A., Obayya, S.S.A.: Fabrication and Characterization of Carbon Nanotubes/PMMA Nanocomposite. Int. Conf. Aerosp. Sci. Aviat. Technol. 15, $1-7$ (2013).

4. Njuguna, J., Pielichowski, K.: Polymer Nanocomposites for Aerospace Applications: Properties. Adv. Eng. Mater. 5, 769-778 (2003).

5. Kiani, Y.: Free vibration of FG-CNT reinforced composite skew plates. Aerosp. Sci. Technol. 58, 178-188 (2016).

6. Naderi Beni, N.: Free vibration analysis of annular sector sandwich plates with FG-CNT reinforced composite face-sheets based on the Carrera's Unified Formulation. Compos. Struct. 214, 269-292 (2019).

7. Wang, Z.X., Shen, H.S.: Nonlinear dynamic response of nanotube-reinforced composite plates resting on elastic foundations in thermal environments. Nonlinear Dyn. 70, 735-754 (2012)

8. Mehar, K., Panda, S.K., Devarajan, Y., Choubey, G.: Numerical Buckling Analysis of Graded CNT-reinforced Composite Sandwich Shell Structure under Thermal Loading. Compos. Struct. 216, 406-414 (2019).

9. Ke, L.L., Yang, J., Kitipornchai, S.: Nonlinear free vibration of functionally graded carbon nanotube-reinforced composite beams. Compos. Struct. 92, 676$683(2010)$ 
10. Lei, Z.X., Liew, K.M., Yu, J.L.: Free vibration analysis of functionally graded carbon nanotube-reinforced composite plates using the element-free kp-Ritz method in thermal environment. Compos. Struct. 106, 128-138 (2013).

11. Zhou, K., Ni, Z., Huang, X., Hua, H.: Stationary/nonstationary stochastic response analysis of composite laminated plates with aerodynamic and thermal loads. Int. J. Mech. Sci. 173, 105461 (2020).

12. Mehar, K., Panda, S.K., Sharma, N.: Numerical investigation and experimental verification of thermal frequency of carbon nanotube-reinforced sandwich structure. Eng. Struct. 211, 110444 (2020).

13. Zhao, W., Païdoussis, M.P., Tang, L., Liu, M., Jiang, J.: Theoretical and experimental investigations of the dynamics of cantilevered flexible plates subjected to axial flow. J. Sound Vib. 331, 575-587 (2012).

14. Kang, D.K., Kim, C.W., Yang, H.I.: Thermal effects on nonlinear vibration of a carbon nanotube-based mass sensor using finite element analysis. Phys. E LowDimensional Syst. Nanostructures. 85, 125-136 (2017).

15. Foroutan, K., Ahmadi, H., Carrera, E.: Nonlinear vibration of imperfect FGCNTRC cylindrical panels under external pressure in the thermal environment. Compos. Struct. 227, 111310 (2019)

16. Shen, H.S., Wang, H.: Nonlinear vibration of shear deformable FGM cylindrical panels resting on elastic foundations in thermal environments. Compos. Part B Eng. 60, 167-177 (2014).

17. Liu, B., Shi, T., Xing, Y.: Three-dimensional free vibration analyses of functionally graded laminated shells under thermal environment by a hierarchical quadrature element method. Compos. Struct. 252, 112733 (2020).

18. Wang, Z.X., Shen, H.S.: Nonlinear vibration of nanotube-reinforced composite plates in thermal environments. Comput. Mater. Sci. 50, 2319-2330 (2011).

19. Fazzolari, F.A.: Thermoelastic vibration and stability of temperature-dependent carbon nanotube-reinforced composite plates. Compos. Struct. 196, 199-214 (2018). 
20. Liu, G., Zhang, W., Xi, A.: Nonlinear Vibrations of Laminated Cross-Ply Composite Cantilever Plate in Subsonic Air Flow. Math. Probl. Eng. 2020, 1-19 (2020).

21. Asadi, H., Souri, M., Wang, Q.: A numerical study on flow-induced instabilities of supersonic FG-CNT reinforced composite flat panels in thermal environments. Compos. Struct. 171, 113-125 (2017).

22. Lin, F., Xiang, Y., Shen, H.S.: Temperature dependent mechanical properties of graphene reinforced polymer nanocomposites-A molecular dynamics simulation. Compos. Part B Eng. 111, 261-269 (2017).

23. Shen, H.S., Xiang, Y., Fan, Y., Hui, D.: Nonlinear bending analysis of FG-GRC laminated cylindrical panels on elastic foundations in thermal environments. Compos. Part B Eng. 141, 148-157 (2018).

24. Shen, H.S., Wang, Z.X.: Nonlinear vibration of hybrid laminated plates resting on elastic foundations in thermal environments. Appl. Math. Model. 36, 62756290 (2012).

25. Han, Y., Elliott, J.: Molecular dynamics simulations of the elastic properties of polymer/carbon nanotube composites. Comput. Mater. Sci. 39, 315-323 (2007).

26. Wang, J.F., Yang, J.P., Tam, L.-h, Zhang, W.: Molecular Dynamics-Based Multiscale Nonlinear Vibration Analysis of PMMA/CNT Composite. Mech. Syst. Signal Process. 153, 107530 (2021).

27. Wang, J.F., Yang, J.P., Tam, L.-h, Zhang, W.: Effect of CNT volume fractions on nonlinear vibration analysis of PMMA/CNT composite plates : a multiscale simulation. Thin-Walled Struct. 170, 108513 (2022)

28. Asadi, H., Beheshti, A.R.: On the nonlinear dynamic responses of FG-CNTRC beams exposed to aerothermal loads using third-order piston theory. Acta Mech, 229(2), 1-18, (2018)

29. Lin, H., Cao, D., Shao, C., Xu, Y.: Studies for aeroelastic characteristics and nonlinear response of FG-CNT reinforced composite panel considering the transient heat conduction. Compos. Struct. 188, 470-482 (2018). 
30. Tersoff, J.: New empirical approach for the structure and energy of covalent systems. Phys. Rev. B. 37, 6991-7000 (1988).

31. Sun, H.: Ab Initio Calculations and Force Field Development for Computer Simulation of Polysilanes. Macromolecules. 28, 701-712 (1995).

32. Arash, B., Park, H.S., Rabczuk, T.: Tensile fracture behavior of short carbon nanotube reinforced polymer composites: A coarse-grained model. Compos. Struct. 134, 981-988 (2015).

33. Zhang, L.W., Ji, W.M., Liew, K.M.: Mechanical properties of diamond nanothread reinforced polymer composites. Carbon. 132, 232-240 (2018).

34. Lu, W., Liu, X., Li, Q., Byun, J.H., Chou, T.W.: Mechanical behavior and structural evolution of carbon nanotube films and fibers under tension: A Coarsegrained molecular dynamics study. J. Appl. Mech. Trans. ASME. 80, 051015 (2013).

35. Usuki, N., Satoh, K., Kamigaito, M.: Synthesis of isotactic-block-syndiotactic poly(methyl methacrylate) via stereospecific living anionic polymerizations in combination with metal-halogen exchange, halogenation, and click reactions. Polymers. 9(12), 723 (2017)

36. Seitz, S., Akashi, M., Ajiro, H.: Pentaerythritol particles covered by layer-bylayer self assembled thin films with stereocomplex of isotactic poly(methyl methacrylate) and syndiotactic poly(methyl methacrylate). Colloid Polym. Sci. 295, 1541-1548 (2017)

37. Al-Taa'y, W.A., Oboudi, S.F., Yousif, E., Abdul Nabi, M., Yusop, R.M., Derawi, D.: Fabrication and Characterization of Nickel Chloride Doped PMMA Films. Adv. Mater. Sci. Eng. 2015, 1-5 (2015).

38. Abdilla, A., Dolinski, N.D., De Roos, P., Ren, J.M., Van Der Woude, E., Seo, S.E., Zayas, M.S., Lawrence, J., Read De Alaniz, J., Hawker, C.J.: Polymer Stereocomplexation as a Scalable Platform for Nanoparticle Assembly. J. Am. Chem. Soc. 142, 1667-1672 (2020)

39. Holding, S.: Polymers: A Property Database. Chroma 72, 587 (2010) 
40. Plimpton, S.: Fast parallel algorithms for short-range molecular dynamics. J. Comput. Phys. 117(1), 1-19 (1995)

41. Hofmann, D., Fritz, L., Ulbrich, J., Schepers, C., Bhning, M.: Detailed-atomistic molecular modeling of small molecule diffusion and solution processes in polymeric membrane materials. Macromol. Theory Simulations. 9, 293-327 (2000)

42. Larsen, G.S., Lin, P., Hart, K.E., Colina, C.M.: Molecular simulations of pim-1like polymers of intrinsic microporosity. Macromolecules. 44, 6944-6951 (2011)

43. Abbott, L.J., Hart, K.E., Colina, C.M.: Polymatic: A generalized simulated polymerization algorithm for amorphous polymers. Theor. Chem. Acc. 132, 119 (2013)

44. Tam, L.-h., Lau, D.: A molecular dynamics investigation on the cross-linking and physical properties of epoxy-based materials. RSC. Adv. 4, 33074-33081 (2014)

45. Soldera, A.: Comparison between the Glass Transition Temperatures of the Two PMMA Tacticities: A Molecular Dynamics Simulation Point of View. Macromol. Symp. 133, 21-32 (1998)

46. Lin, L., Kedzierski, M.A.: Density and viscosity of a polyol ester lubricant: Measurement and molecular dynamics simulation. Int. J. Refrig. 118, 188-201 (2020).

47. Levitas, V.I., Ravelo, R.: Virtual melting as a new mechanism of stress relaxation under high strain rate loading. Proc. Natl. Acad. Sci. U. S. A. 109, 13204-13207 (2012).

48. Nosé, S.: A unified formulation of the constant temperature molecular dynamics methods. J. Chem. Phys. 81, 511-519 (1984)

49. Andersen, H.C.: Molecular dynamics simulations at constant pressure and/or temperature. J. Chem. Phys. 72(4), 2384-2393 (1980)

50. García-Macías, E., Guzmán, C.F., Saavedra Flores, E.I., Castro-Triguero, R.: 
Multiscale modeling of the elastic moduli of CNT-reinforced polymers and fitting of efficiency parameters for the use of the extended rule-of-mixtures. Compos. Part B Eng. 159, 114-131 (2019).

51. M.Y.B. Elshabasy, M., Kouritem, S.A.: Thickening of optimally selected locations on panels subjected to unyawed flow for substantial delay of the panel flutter. Alexandria Eng. J. 59, 5031-5044 (2020).

52. Zhou, R.C., Mei, C., Huang, J.K.: Suppression of nonlinear panel flutter at supersonic speeds and elevated temperatures. AIAA J. 34(2), 347-354 (1996).

53. Abdel-Motagaly, K., Guo, X., Duan, B., Mei, C.: Active control of nonlinear panel flutter under yawed supersonic flow. AIAA J. 43(3), 671-680 (2005).

54. Guenat, E., Schiffmann, J.: Real-gas effects on aerodynamic bearings. Tribol. Int. 120, 358-368 (2018).

55. Bauman, R.P., Schwaneberg, R.: Interpretation of Bernoulli's equation. Phys. Teach. 32, 478-488 (1994).

56. J.N.Reddy: Mechanics of laminated composite plates and shells:theory and analysis. (2003)

57. Zhou, M., Xiong, X., Drummer, D., Jiang, B.: Molecular Dynamics Simulation on the Effect of Bonding Pressure on Thermal Bonding of Polymer Microfluidic Chip. Polymers. 11, 557 (2019)

58. Fedele, L., Marinetti, S., Bobbo, S., Scattolini, M.: PpT experimental measurements and data correlation of pentaerythritol esters. J. Chem. Eng. Data. $52,108-115$ (2007).

59. Prolongo, S.G., Campo, M., Gude, M.R., Chaos-Morán, R., Ureña, A.: Thermophysical characterisation of epoxy resin reinforced by amino-functionalized carbon nanofibers. Compos. Sci. Technol. 69, 349-357 (2009)

60. Tam, L.-h., Wu, C.: Molecular Mechanics of the Moisture Effect on Epoxy/Carbon Nanotube Nanocomposites. Nanomaterials. 7, 324 (2017)

61. Shen, H.S., Xiang, Y.: Nonlinear bending of nanotube-reinforced composite cylindrical panels resting on elastic foundations in thermal environments. Eng. 
Struct. 80, 163-172 (2014).

62. Li, M., Deng, T., Zheng, B., Zhang, Y., Liao, Y., Zhou, H.: Effect of defects on the mechanical and thermal properties of graphene. Nanomaterials. 9, (2019).

63. Hadipeykani, M., Aghadavoudi, F., Toghraie, D.: A molecular dynamics simulation of the glass transition temperature and volumetric thermal expansion coefficient of thermoset polymer based epoxy nanocomposite reinforced by CNT: A statistical study. Phys. A Stat. Mech. its Appl. 546, 123995 (2020).

64. Shirasu, K., Yamamoto, G., Tamaki, I., Ogasawara, T., Shimamura, Y., Inoue, Y., Hashida, T.: Negative axial thermal expansion coefficient of carbon nanotubes: Experimental determination based on measurements of coefficient of thermal expansion for aligned carbon nanotube reinforced epoxy composites. Carbon. 95, 904-909 (2015).

65. Hassanzadeh-Aghdam, M.K., Ansari, R., Darvizeh, A.: Thermal expanding behavior of carbon nanotube-shape memory polymer nanocomposites. Mech. Adv. Mater. Struct. 26, 1858-1869 (2019).

66. Moore, D.R.: Mechanical properties of polymers and composites. Polymer. 16(7), 552-552 (1975).

67. Wang, Q., Liew, K.M.: Mechanical properties of carbon nanotubes. Carbon Nanotub. New Res. 260, 157-174 (2009).

68. Berrahou, N., Mokaddem, A., Doumi, B., Hiadsi, S., Beldjoudi, N., Boutaous, A.: Investigation by molecular dynamics simulation of the glass transition temperature and elastic properties of amorphous polymers PMMA, PMAAM and PMMA co PMAAM copolymers. Polym. Bull. 73, 3007-3017 (2016)

69. David, O. Ben, Banks-Sills, L., Aboudi, J., Fourman, V., Eliasi, R., Simhi, T., Shlayer, A., Raz, O.: Evaluation of the Mechanical Properties of PMMA Reinforced with Carbon Nanotubes-Experiments and Modeling. Exp. Mech. 54, 175-186 (2014).

70. Chen, X., Wang, X., Liu, B.Y.: Effect of temperature on elastic properties of single-walled carbon nanotubes. J. Reinf. Plast. Compos. 28, 551-569 (2009). 
71. Turagam, N., Prasad Mudrakola, D.: Effect of Micro-Additions of Carbon Nanotubes to Polymethylmethacrylate on Reduction in Polymerization Shrinkage. J. Prosthodont. 22(2), 105-111 (2013).

72. Buehler, M.J.: Mesoscale modelling of mechanics of carbon nanotubes: Selfassembly, self-folding, and fracture. J. Mater. Res. 21, 2855-2869 (2006)

73. Khoury, E. El, Messager, T., Cartraud, P., Khoury, E. El, Messager, T., Cartraud, P.: Derivation of the Young's and shear moduli of single-walled carbon nanotubes through a computational homogenization approach. Int. J. Multiscale Comput. Eng. 9 (1), 97-118 (2011)

74. To, C.W.S.: Bending and shear moduli of single-walled carbon nanotubes. Finite Elem. Anal. Des. 42, 404-413 (2006).

75. Sahputra, I.H., Alexiadis, A., Adams, M.J.: Temperature and configurational effects on the Young's modulus of poly (methyl methacrylate): a molecular dynamics study comparing the DREIDING, AMBER and OPLS force fields. Mol. Simul. 44, 774-780 (2018).

76. Yang, S., Yu, S., Kyoung, W., Han, D.S., Cho, M.: Multiscale modeling of sizedependent elastic properties of carbon nanotube/polymer nanocomposites with interfacial imperfections. Polymer. 53, 623-633 (2012)

77. Maglieri, D., Tinetti, A., Henderson, H.: Measured Sonic Boom Signatures Above and Below the XB-70 Airplane Flying at Mach Number 1.5 and 37,000 Feet. National Aeronautics and Space Administration (2011)

78. Liu, Y.Z., Hao, Y.X., Zhang, W., Chen, J., Li, S.B.: Nonlinear dynamics of initially imperfect functionally graded circular cylindrical shell under complex loads. J. Sound Vib. 348, 294-328 (2015).

\section{Appendix Expressions of the equations of motion}

$$
\begin{aligned}
N_{\mathrm{x}}= & A_{11}\left(\frac{1}{2} \frac{\partial^{2} w_{0}}{\partial x^{2}}+\frac{\partial u_{0}}{\partial x}\right)+A_{12}\left(\frac{1}{2} \frac{\partial^{2} w_{0}}{\partial y^{2}}+\frac{\partial v_{0}}{\partial y}\right)+A_{16}\left(\frac{\partial w_{0}}{\partial x} \frac{\partial w_{0}}{\partial y}+\frac{\partial u_{0}}{\partial y}+\frac{\partial v_{0}}{\partial x}\right)+B_{11} \frac{\partial \phi_{\mathrm{x}}}{\partial x}+B_{12} \frac{\partial \phi_{\mathrm{y}}}{\partial y} \\
& +B_{16}\left(\frac{\partial \phi_{\mathrm{y}}}{\partial x}+\frac{\partial \phi_{\mathrm{x}}}{\partial y}\right)-c_{1} E_{11}\left(\frac{\partial \phi_{\mathrm{x}}}{\partial x}+\frac{\partial^{2} w_{0}}{\partial x^{2}}\right)-c_{1} E_{12}\left(\frac{\partial \phi_{\mathrm{y}}}{\partial y}+\frac{\partial^{2} w_{0}}{\partial y^{2}}\right)-c_{1} E_{16}\left(\frac{\partial \phi_{\mathrm{y}}}{\partial x}+\frac{\partial \phi_{\mathrm{x}}}{\partial y}+2 \frac{\partial^{2} w_{0}}{\partial x \partial y}\right)
\end{aligned}
$$




$$
\begin{aligned}
& -N_{\mathrm{x}}^{T} \\
& N_{\mathrm{xy}}=A_{16}\left(\frac{1}{2} \frac{\partial^{2} w_{0}}{\partial x^{2}}+\frac{\partial u_{0}}{\partial x}\right)+A_{26}\left(\frac{1}{2} \frac{\partial^{2} w_{0}}{\partial y^{2}}+\frac{\partial v_{0}}{\partial y}\right)+A_{66}\left(\frac{\partial w_{0}}{\partial x} \frac{\partial w_{0}}{\partial y}+\frac{\partial u_{0}}{\partial y}+\frac{\partial v_{0}}{\partial x}\right)+B_{16} \frac{\partial \phi_{\mathrm{x}}}{\partial x}+B_{26} \frac{\partial \phi_{\mathrm{y}}}{\partial y} \\
& +B_{66}\left(\frac{\partial \phi_{\mathrm{y}}}{\partial x}+\frac{\partial \phi_{\mathrm{x}}}{\partial y}\right)-c_{1} E_{16}\left(\frac{\partial \phi_{\mathrm{x}}}{\partial x}+\frac{\partial w_{0}}{\partial x^{2}}\right)-c_{1} E_{26}\left(\frac{\partial \phi_{\mathrm{y}}}{\partial y}+\frac{\partial^{2} w_{0}}{\partial y^{2}}\right) \\
& -c_{1} E_{66}\left(\frac{\partial \phi_{\mathrm{y}}}{\partial x}+\frac{\partial \phi_{\mathrm{x}}}{\partial y}+2 \frac{\partial^{2} w_{0}}{\partial x \partial y}\right) \\
& N_{\mathrm{y}}=A_{12}\left(\frac{1}{2} \frac{\partial^{2} w_{0}}{\partial x^{2}}+\frac{\partial u_{0}}{\partial x}\right)+A_{22}\left(\frac{1}{2} \frac{\partial^{2} w_{0}}{\partial y^{2}}+\frac{\partial v_{0}}{\partial y}\right)+A_{26}\left(\frac{\partial w_{0}}{\partial x} \frac{\partial w_{0}}{\partial y}+\frac{\partial u_{0}}{\partial y}+\frac{\partial v_{0}}{\partial x}\right)+B_{12} \frac{\partial \phi_{\mathrm{x}}}{\partial x}+B_{22} \frac{\partial \phi_{\mathrm{y}}}{\partial y} \\
& +B_{26}\left(\frac{\partial \phi_{\mathrm{y}}}{\partial x}+\frac{\partial \phi_{\mathrm{x}}}{\partial y}\right)-c_{1} E_{12}\left(\frac{\partial \phi_{\mathrm{x}}}{\partial x}+\frac{\partial^{2} w_{0}}{\partial x^{2}}\right)-c_{1} E_{22}\left(\frac{\partial \phi_{\mathrm{y}}}{\partial y}+\frac{\partial^{2} w_{0}}{\partial y^{2}}\right)-c_{1} E_{26}\left(\frac{\partial \phi_{\mathrm{y}}}{\partial x}+\frac{\partial \phi_{\mathrm{x}}}{\partial y}\right. \\
& \left.+2 \frac{\partial^{2} w_{0}}{\partial x \partial y}\right)-N_{\mathrm{y}}^{T} \\
& \frac{\partial N_{\mathrm{x}}}{\partial x}=A_{11}\left(\frac{\partial w_{0}}{\partial x} \frac{\partial^{2} w_{0}}{\partial x^{2}}+\frac{\partial^{2} u_{0}}{\partial x^{2}}\right)+A_{12}\left(\frac{\partial^{2} w_{0}}{\partial x \partial y} \frac{\partial w_{0}}{\partial y}+\frac{\partial^{2} v_{0}}{\partial x \partial y}\right)+A_{16}\left(\frac{\partial w_{0}}{\partial x} \frac{\partial^{2} w_{0}}{\partial x \partial y}+\frac{\partial^{2} w_{0}}{\partial x^{2}} \frac{\partial w_{0}}{\partial y}+\frac{\partial^{2} u_{0}}{\partial x \partial y}+\frac{\partial^{2} v_{0}}{\partial x^{2}}\right) \\
& +B_{11} \frac{\partial^{2} \phi_{\mathrm{x}}}{\partial x^{2}}+B_{12} \frac{\partial^{2} \phi_{\mathrm{y}}}{\partial x \partial y}+B_{16}\left(\frac{\partial^{2} \phi_{\mathrm{y}}}{\partial x^{2}}+\frac{\partial^{2} \phi_{\mathrm{x}}}{\partial x \partial y}\right)-c_{1} E_{11}\left(\frac{\partial^{2} \phi_{\mathrm{x}}}{\partial x^{2}}+\frac{\partial^{3} w_{0}}{\partial x^{3}}\right)-c_{1} E_{12}\left(\frac{\partial^{2} \phi_{\mathrm{y}}}{\partial x \partial y}\right. \\
& \left.+\frac{\partial^{3} w_{0}}{\partial x \partial y^{2}}\right)-c_{1} E_{16}\left(\frac{\partial^{2} \phi_{\mathrm{y}}}{\partial x^{2}}+\frac{\partial^{2} \phi_{\mathrm{x}}}{\partial x \partial y}+2 \frac{\partial^{3} w_{0}}{\partial x^{2} \partial y}\right)-\frac{\partial N_{\mathrm{x}}^{T}}{\partial x} \\
& \frac{\partial N_{\mathrm{xy}}}{\partial y}=A_{16}\left(\frac{\partial w_{0}}{\partial x} \frac{\partial^{2} w_{0}}{\partial x \partial y}+\frac{\partial^{2} u_{0}}{\partial x \partial y}\right)+A_{26}\left(\frac{\partial w_{0}}{\partial y} \frac{\partial^{2} w_{0}}{\partial y^{2}}+\frac{\partial^{2} v_{0}}{\partial y^{2}}\right)+A_{66}\left(\frac{\partial w_{0}}{\partial x} \frac{\partial^{2} w_{0}}{\partial y^{2}}+\frac{\partial^{2} w_{0}}{\partial x \partial y} \frac{\partial w_{0}}{\partial y}+\frac{\partial^{2} u_{0}}{\partial y^{2}}+\frac{\partial^{2} v_{0}}{\partial x \partial y}\right) \\
& +B_{16} \frac{\partial^{2} \phi_{\mathrm{x}}}{\partial x \partial y}+B_{26} \frac{\partial^{2} \phi_{\mathrm{y}}}{\partial y^{2}}+B_{66}\left(\frac{\partial^{2} \phi_{\mathrm{y}}}{\partial x \partial y}+\frac{\partial^{2} \phi_{\mathrm{x}}}{\partial y^{2}}\right)-c_{1} E_{16}\left(\frac{\partial^{2} \phi_{\mathrm{x}}}{\partial x \partial y}+\frac{\partial^{3} w_{0}}{\partial x^{2} \partial y}\right)-c_{1} E_{26}\left(\frac{\partial^{2} \phi_{\mathrm{y}}}{\partial y^{2}}+\frac{\partial^{3} w_{0}}{\partial y^{3}}\right) \\
& -c_{1} E_{66}\left(\frac{\partial^{2} \phi_{\mathrm{y}}}{\partial x \partial y}+\frac{\partial^{2} \phi_{\mathrm{x}}}{\partial y^{2}}+2 \frac{\partial^{3} w_{0}}{\partial x \partial y^{2}}\right) \\
& \frac{\partial N_{\mathrm{y}}}{\partial y}=A_{12}\left(\frac{\partial w_{0}}{\partial x} \frac{\partial^{2} w_{0}}{\partial x \partial y}+\frac{\partial^{2} u_{0}}{\partial x \partial y}\right)+A_{22}\left(\frac{\partial^{2} w_{0}}{\partial y^{2}} \frac{\partial w_{0}}{\partial y}+\frac{\partial^{2} v_{0}}{\partial y^{2}}\right)+A_{26}\left(\frac{\partial w_{0}}{\partial x} \frac{\partial^{2} w_{0}}{\partial y^{2}}+\frac{\partial^{2} w_{0}}{\partial x \partial y} \frac{\partial w_{0}}{\partial y}+\frac{\partial^{2} u_{0}}{\partial y^{2}}+\frac{\partial^{2} v_{0}}{\partial x \partial y}\right) \\
& +B_{12} \frac{\partial^{2} \phi_{\mathrm{x}}}{\partial x \partial y}+B_{22} \frac{\partial^{2} \phi_{\mathrm{y}}}{\partial y^{2}}+B_{26}\left(\frac{\partial^{2} \phi_{\mathrm{y}}}{\partial x \partial y}+\frac{\partial^{2} \phi_{\mathrm{x}}}{\partial y^{2}}\right)-c_{1} E_{12}\left(\frac{\partial^{2} \phi_{\mathrm{x}}}{\partial x \partial y}+\frac{\partial^{3} w_{0}}{\partial x^{2} \partial y}\right)-c_{1} E_{22}\left(\frac{\partial^{2} \phi_{\mathrm{y}}}{\partial y^{2}}+\frac{\partial^{3} w_{0}}{\partial y^{3}}\right)
\end{aligned}
$$




$$
\begin{aligned}
& -c_{1} E_{26}\left(\frac{\partial^{2} \phi_{\mathrm{y}}}{\partial x \partial y}+\frac{\partial^{2} \phi_{\mathrm{x}}}{\partial y^{2}}+2 \frac{\partial^{3} w_{0}}{\partial x \partial y^{2}}\right)-\frac{\partial N_{\mathrm{y}}^{T}}{\partial y} \\
& \frac{\partial N_{\mathrm{xy}}}{\partial x}=A_{16}\left(\frac{\partial w_{0}}{\partial x} \frac{\partial^{2} w_{0}}{\partial x^{2}}+\frac{\partial^{2} u_{0}}{\partial x^{2}}\right)+A_{26}\left(\frac{\partial w_{0}}{\partial y} \frac{\partial^{2} w_{0}}{\partial x \partial y}+\frac{\partial^{2} v_{0}}{\partial x \partial y}\right)+A_{66}\left(\frac{\partial w_{0}}{\partial x} \frac{\partial^{2} w_{0}}{\partial x \partial y}+\frac{\partial^{2} w_{0}}{\partial x^{2}} \frac{\partial w_{0}}{\partial y}+\frac{\partial^{2} u_{0}}{\partial x \partial y}+\frac{\partial^{2} v_{0}}{\partial x^{2}}\right) \\
& +B_{16} \frac{\partial^{2} \phi_{\mathrm{x}}}{\partial x^{2}}+B_{26} \frac{\partial^{2} \phi_{\mathrm{y}}}{\partial x \partial y}+B_{66}\left(\frac{\partial^{2} \phi_{\mathrm{y}}}{\partial x^{2}}+\frac{\partial^{2} \phi_{\mathrm{x}}}{\partial x \partial y}\right)-c_{1} E_{16}\left(\frac{\partial^{2} \phi_{\mathrm{x}}}{\partial x^{2}}+\frac{\partial^{3} w_{0}}{\partial x^{3}}\right)-c_{1} E_{26}\left(\frac{\partial^{2} \phi_{\mathrm{y}}}{\partial x \partial y}+\frac{\partial^{3} w_{0}}{\partial x \partial y^{2}}\right) \\
& -c_{1} E_{66}\left(\frac{\partial^{2} \phi_{\mathrm{y}}}{\partial x^{2}}+\frac{\partial^{2} \phi_{\mathrm{x}}}{\partial x \partial y}+2 \frac{\partial^{3} w_{0}}{\partial x^{2} \partial y}\right) \\
& Q_{\mathrm{xz}}=A_{55}\left(\phi_{\mathrm{x}}+\frac{\partial w_{0}}{\partial x}\right)-c_{2} D_{55}\left(\phi_{\mathrm{x}}+\frac{\partial w_{0}}{\partial x}\right) \\
& \frac{\partial Q_{\mathrm{xz}}}{\partial x}=A_{55}\left(\frac{\partial \phi_{\mathrm{x}}}{\partial x}+\frac{\partial^{2} w_{0}}{\partial x^{2}}\right)-c_{2} D_{55}\left(\frac{\partial \phi_{\mathrm{x}}}{\partial x}+\frac{\partial^{2} w_{0}}{\partial x^{2}}\right), \\
& Q_{\mathrm{yz}}=A_{44}\left(\phi_{\mathrm{y}}+\frac{\partial w_{0}}{\partial y}\right)-c_{2} D_{44}\left(\phi_{\mathrm{y}}+\frac{\partial w_{0}}{\partial y}\right) \\
& \frac{\partial Q_{\mathrm{yz}}}{\partial y}=A_{44}\left(\frac{\partial \phi_{\mathrm{y}}}{\partial y}+\frac{\partial^{2} w_{0}}{\partial y^{2}}\right)-c_{2} D_{44}\left(\frac{\partial \phi_{\mathrm{y}}}{\partial y}+\frac{\partial^{2} w_{0}}{\partial y^{2}}\right) \\
& R_{\mathrm{xz}}=D_{55}\left(\phi_{\mathrm{x}}+\frac{\partial w_{0}}{\partial x}\right)-c_{2} F_{55}\left(\phi_{\mathrm{x}}+\frac{\partial w_{0}}{\partial x}\right) \\
& \frac{\partial R_{\mathrm{xz}}}{\partial x}=D_{55}\left(\frac{\partial \phi_{\mathrm{x}}}{\partial x}+\frac{\partial^{2} w_{0}}{\partial x^{2}}\right)-c_{2} F_{55}\left(\frac{\partial \phi_{\mathrm{x}}}{\partial x}+\frac{\partial^{2} w_{0}}{\partial x^{2}}\right) \\
& R_{\mathrm{yz}}=D_{44}\left(\phi_{\mathrm{y}}+\frac{\partial w_{0}}{\partial y}\right)-c_{2} F_{44}\left(\phi_{\mathrm{y}}+\frac{\partial w_{0}}{\partial y}\right) \\
& \frac{\partial R_{\mathrm{yz}}}{\partial y}=D_{44}\left(\frac{\partial \phi_{\mathrm{y}}}{\partial y}+\frac{\partial^{2} w_{0}}{\partial y^{2}}\right)-c_{2} F_{44}\left(\frac{\partial \phi_{\mathrm{y}}}{\partial y}+\frac{\partial^{2} w_{0}}{\partial y^{2}}\right) \\
& \frac{\partial P_{\mathrm{x}}}{\partial x}=E_{11}\left(\frac{\partial w_{0}}{\partial x} \frac{\partial^{2} w_{0}}{\partial x^{2}}+\frac{\partial^{2} u_{0}}{\partial x^{2}}\right)+E_{12}\left(\frac{\partial^{2} w_{0}}{\partial x \partial y} \frac{\partial w_{0}}{\partial y}+\frac{\partial^{2} v_{0}}{\partial x \partial y}\right)+E_{16}\left(\frac{\partial w_{0}}{\partial x} \frac{\partial^{2} w_{0}}{\partial x \partial y}+\frac{\partial^{2} w_{0}}{\partial x^{2}} \frac{\partial w_{0}}{\partial y}+\frac{\partial^{2} u_{0}}{\partial x \partial y}+\frac{\partial^{2} v_{0}}{\partial x^{2}}\right) \\
& +F_{11} \frac{\partial^{2} \phi_{\mathrm{x}}}{\partial x^{2}}+F_{12} \frac{\partial^{2} \phi_{\mathrm{y}}}{\partial x \partial y}+F_{16}\left(\frac{\partial^{2} \phi_{\mathrm{y}}}{\partial x^{2}}+\frac{\partial^{2} \phi_{\mathrm{x}}}{\partial x \partial y}\right)-c_{1} H_{11}\left(\frac{\partial^{2} \phi_{\mathrm{x}}}{\partial x^{2}}+\frac{\partial^{3} w_{0}}{\partial x^{3}}\right)-c_{1} H_{12}\left(\frac{\partial^{2} \phi_{\mathrm{y}}}{\partial x \partial y}+\frac{\partial^{3} w_{0}}{\partial x \partial y^{2}}\right)
\end{aligned}
$$




$$
\begin{aligned}
& -c_{1} H_{16}\left(\frac{\partial^{2} \phi_{\mathrm{y}}}{\partial x^{2}}+\frac{\partial^{2} \phi_{\mathrm{x}}}{\partial x \partial y}+2 \frac{\partial^{3} w_{0}}{\partial x^{2} \partial y}\right)-\frac{\partial P_{\mathrm{x}}^{T}}{\partial x} \\
& \frac{\partial^{2} P_{\mathrm{x}}}{\partial x^{2}}=E_{11}\left(\frac{\partial^{2} w_{0}}{\partial x^{2}} \frac{\partial^{2} w_{0}}{\partial x^{2}}+\frac{\partial w_{0}}{\partial x} \frac{\partial^{3} w_{0}}{\partial x^{3}}+\frac{\partial^{3} u_{0}}{\partial x^{3}}\right)+E_{12}\left(\frac{\partial^{3} w_{0}}{\partial x^{2} \partial y} \frac{\partial w_{0}}{\partial y}+\frac{\partial^{2} w_{0}}{\partial x \partial y} \frac{\partial^{2} w_{0}}{\partial x \partial y}+\frac{\partial^{3} v_{0}}{\partial x^{2} \partial y}\right) \\
& +E_{16}\left(\frac{\partial w_{0}}{\partial x} \frac{\partial^{3} w_{0}}{\partial x^{2} \partial y}+\frac{\partial^{3} w_{0}}{\partial x^{3}} \frac{\partial w_{0}}{\partial y}+2 \frac{\partial^{2} w_{0}}{\partial x^{2}} \frac{\partial^{2} w_{0}}{\partial x \partial y}+\frac{\partial^{3} u_{0}}{\partial x^{2} \partial y}+\frac{\partial^{3} v_{0}}{\partial x^{3}}\right)+F_{11} \frac{\partial^{3} \phi_{\mathrm{x}}}{\partial x^{3}}+F_{12} \frac{\partial^{3} \phi_{\mathrm{y}}}{\partial x^{2} \partial y} \\
& +F_{16}\left(\frac{\partial^{3} \phi_{\mathrm{y}}}{\partial x^{3}}+\frac{\partial^{3} \phi_{\mathrm{x}}}{\partial x^{2} \partial y}\right)-c_{1} H_{11}\left(\frac{\partial^{3} \phi_{\mathrm{x}}}{\partial x^{3}}+\frac{\partial^{4} w_{0}}{\partial x^{4}}\right)-c_{1} H_{12}\left(\frac{\partial^{3} \phi_{\mathrm{y}}}{\partial x^{2} \partial y}+\frac{\partial^{4} w_{0}}{\partial x^{2} \partial y^{2}}\right) \\
& -c_{1} H_{16}\left(\frac{\partial^{3} \phi_{\mathrm{y}}}{\partial x^{3}}+\frac{\partial^{3} \phi_{\mathrm{x}}}{\partial x^{2} \partial y}+2 \frac{\partial^{4} w_{0}}{\partial x^{3} \partial y}\right)-\frac{\partial^{2} P_{\mathrm{x}}^{T}}{\partial x^{2}} \\
& \frac{\partial P_{\mathrm{xy}}}{\partial y}=E_{16}\left(\frac{\partial w_{0}}{\partial x} \frac{\partial^{2} w_{0}}{\partial x \partial y}+\frac{\partial^{2} u_{0}}{\partial x \partial y}\right)+E_{26}\left(\frac{\partial w_{0}}{\partial y} \frac{\partial^{2} w_{0}}{\partial y^{2}}+\frac{\partial^{2} v_{0}}{\partial y^{2}}\right)+E_{66}\left(\frac{\partial w_{0}}{\partial x} \frac{\partial^{2} w_{0}}{\partial y^{2}}+\frac{\partial^{2} w_{0}}{\partial x \partial y} \frac{\partial w_{0}}{\partial y}+\frac{\partial^{2} u_{0}}{\partial y^{2}}+\frac{\partial^{2} v_{0}}{\partial x \partial y}\right) \\
& +F_{16} \frac{\partial^{2} \phi_{\mathrm{x}}}{\partial x \partial y}+F_{26} \frac{\partial^{2} \phi_{\mathrm{y}}}{\partial y^{2}}+F_{66}\left(\frac{\partial^{2} \phi_{\mathrm{y}}}{\partial x \partial y}+\frac{\partial^{2} \phi_{\mathrm{x}}}{\partial y^{2}}\right)-c_{1} H_{16}\left(\frac{\partial^{2} \phi_{\mathrm{x}}}{\partial x \partial y}+\frac{\partial^{3} w_{0}}{\partial x^{2} \partial y}\right)-c_{1} H_{26}\left(\frac{\partial^{2} \phi_{\mathrm{y}}}{\partial y^{2}}+\frac{\partial^{3} w_{0}}{\partial y^{3}}\right) \\
& -c_{1} H_{66}\left(\frac{\partial^{2} \phi_{\mathrm{y}}}{\partial x \partial y}+\frac{\partial^{2} \phi_{\mathrm{x}}}{\partial y^{2}}+2 \frac{\partial^{3} w_{0}}{\partial x \partial y^{2}}\right) \\
& \frac{\partial P_{\mathrm{y}}}{\partial y}=E_{12}\left(\frac{\partial w_{0}}{\partial x} \frac{\partial^{2} w_{0}}{\partial x \partial y}+\frac{\partial^{2} u_{0}}{\partial x \partial y}\right)+E_{22}\left(\frac{\partial^{2} w_{0}}{\partial y^{2}} \frac{\partial w_{0}}{\partial y}+\frac{\partial^{2} v_{0}}{\partial y^{2}}\right)+E_{26}\left(\frac{\partial w_{0}}{\partial x} \frac{\partial^{2} w_{0}}{\partial y^{2}}+\frac{\partial^{2} w_{0}}{\partial x \partial y} \frac{\partial w_{0}}{\partial y}+\frac{\partial^{2} u_{0}}{\partial y^{2}}+\frac{\partial^{2} v_{0}}{\partial x \partial y}\right) \\
& +F_{12} \frac{\partial^{2} \phi_{\mathrm{x}}}{\partial x \partial y}+F_{22} \frac{\partial^{2} \phi_{\mathrm{y}}}{\partial y^{2}}+F_{26}\left(\frac{\partial^{2} \phi_{\mathrm{y}}}{\partial x \partial y}+\frac{\partial^{2} \phi_{\mathrm{x}}}{\partial y^{2}}\right)-c_{1} H_{12}\left(\frac{\partial^{2} \phi_{\mathrm{x}}}{\partial x \partial y}+\frac{\partial^{3} w_{0}}{\partial x^{2} \partial y}\right)-c_{1} H_{22}\left(\frac{\partial^{2} \phi_{\mathrm{y}}}{\partial y^{2}}+\frac{\partial^{3} w_{0}}{\partial y^{3}}\right) \\
& -c_{1} H_{26}\left(\frac{\partial^{2} \phi_{\mathrm{y}}}{\partial x \partial y}+\frac{\partial^{2} \phi_{\mathrm{x}}}{\partial y^{2}}+2 \frac{\partial^{3} w_{0}}{\partial x \partial y^{2}}\right)-\frac{\partial P_{\mathrm{y}}^{T}}{\partial y} \\
& \frac{\partial^{2} P_{\mathrm{y}}}{\partial y^{2}}=E_{12}\left(\frac{\partial^{2} w_{0}}{\partial x \partial y} \frac{\partial^{2} w_{0}}{\partial x \partial y}+\frac{\partial w_{0}}{\partial x} \frac{\partial^{3} w_{0}}{\partial x \partial y^{2}}+\frac{\partial^{3} u_{0}}{\partial x \partial y^{2}}\right)+E_{22}\left(\frac{\partial^{3} w_{0}}{\partial y^{3}} \frac{\partial w_{0}}{\partial y}+\frac{\partial^{2} w_{0}}{\partial y^{2}} \frac{\partial^{2} w_{0}}{\partial y^{2}}+\frac{\partial^{3} v_{0}}{\partial y^{3}}\right) \\
& +E_{26}\left(\frac{\partial w_{0}}{\partial x} \frac{\partial^{3} w_{0}}{\partial y^{3}}+\frac{\partial^{3} w_{0}}{\partial x \partial y^{2}} \frac{\partial w_{0}}{\partial y}+2 \frac{\partial^{2} w_{0}}{\partial x \partial y} \frac{\partial^{2} w_{0}}{\partial y^{2}}+\frac{\partial^{3} u_{0}}{\partial y^{3}}+\frac{\partial^{3} v_{0}}{\partial x \partial y^{2}}\right)+F_{12} \frac{\partial^{3} \phi_{\mathrm{x}}}{\partial x \partial y^{2}}+F_{22} \frac{\partial^{3} \phi_{\mathrm{y}}}{\partial y^{3}} \\
& +F_{26}\left(\frac{\partial^{3} \phi_{\mathrm{y}}}{\partial x \partial y^{2}}+\frac{\partial^{3} \phi_{\mathrm{x}}}{\partial y^{3}}\right)-c_{1} H_{12}\left(\frac{\partial^{3} \phi_{\mathrm{x}}}{\partial x \partial y^{2}}+\frac{\partial^{4} w_{0}}{\partial x^{2} \partial y^{2}}\right)-c_{1} H_{22}\left(\frac{\partial^{3} \phi_{\mathrm{y}}}{\partial y^{3}}+\frac{\partial^{4} w_{0}}{\partial y^{4}}\right)
\end{aligned}
$$




$$
\begin{aligned}
& -c_{1} H_{26}\left(\frac{\partial^{3} \phi_{\mathrm{y}}}{\partial x \partial y^{2}}+\frac{\partial^{3} \phi_{\mathrm{x}}}{\partial y^{3}}+2 \frac{\partial^{4} w_{0}}{\partial x \partial y^{3}}\right)-\frac{\partial^{2} P_{\mathrm{y}}^{T}}{\partial y^{2}} \\
& \frac{\partial P_{\mathrm{xy}}}{\partial x}=E_{16}\left(\frac{\partial w_{0}}{\partial x} \frac{\partial^{2} w_{0}}{\partial x^{2}}+\frac{\partial^{2} u_{0}}{\partial x^{2}}\right)+E_{26}\left(\frac{\partial w_{0}}{\partial y} \frac{\partial^{2} w_{0}}{\partial x \partial y}+\frac{\partial^{2} v_{0}}{\partial x \partial y}\right)+E_{66}\left(\frac{\partial w_{0}}{\partial x} \frac{\partial^{2} w_{0}}{\partial x \partial y}+\frac{\partial^{2} w_{0}}{\partial x^{2}} \frac{\partial w_{0}}{\partial y}+\frac{\partial^{2} u_{0}}{\partial x \partial y}+\frac{\partial^{2} v_{0}}{\partial x^{2}}\right) \\
& +F_{16} \frac{\partial^{2} \phi_{\mathrm{x}}}{\partial x^{2}}+F_{26} \frac{\partial^{2} \phi_{\mathrm{y}}}{\partial x \partial y}+F_{66}\left(\frac{\partial^{2} \phi_{\mathrm{y}}}{\partial x^{2}}+\frac{\partial^{2} \phi_{\mathrm{x}}}{\partial x \partial y}\right)-c_{1} H_{16}\left(\frac{\partial^{2} \phi_{\mathrm{x}}}{\partial x^{2}}+\frac{\partial^{3} w_{0}}{\partial x^{3}}\right)-c_{1} H_{26}\left(\frac{\partial^{2} \phi_{\mathrm{y}}}{\partial x \partial y}+\frac{\partial^{3} w_{0}}{\partial x \partial y^{2}}\right) \\
& -c_{1} H_{66}\left(\frac{\partial^{2} \phi_{\mathrm{y}}}{\partial x^{2}}+\frac{\partial^{2} \phi_{\mathrm{x}}}{\partial x \partial y}+2 \frac{\partial^{3} w_{0}}{\partial x^{2} \partial y}\right) \\
& \frac{\partial^{2} P_{\mathrm{xy}}}{\partial x \partial y}=E_{16}\left(\frac{\partial^{2} w_{0}}{\partial x \partial y} \frac{\partial^{2} w_{0}}{\partial x^{2}}+\frac{\partial w_{0}}{\partial x} \frac{\partial^{3} w_{0}}{\partial x^{2} \partial y}+\frac{\partial^{3} u_{0}}{\partial x^{2} \partial y}\right)+E_{26}\left(\frac{\partial^{2} w_{0}}{\partial y^{2}} \frac{\partial^{2} w_{0}}{\partial x \partial y}+\frac{\partial w_{0}}{\partial y} \frac{\partial^{3} w_{0}}{\partial x \partial y^{2}}+\frac{\partial^{3} v_{0}}{\partial x \partial y^{2}}\right) \\
& +E_{66}\left(\frac{\partial^{2} w_{0}}{\partial x \partial y} \frac{\partial^{2} w_{0}}{\partial x \partial y}+\frac{\partial w_{0}}{\partial x} \frac{\partial^{3} w_{0}}{\partial x \partial y^{2}}+\frac{\partial^{3} w_{0}}{\partial x^{2} \partial y} \frac{\partial w_{0}}{\partial y}+\frac{\partial^{2} w_{0}}{\partial x^{2}} \frac{\partial^{2} w_{0}}{\partial y^{2}}+\frac{\partial^{3} u_{0}}{\partial x \partial y^{2}}+\frac{\partial^{3} v_{0}}{\partial x^{2} \partial y}\right) \\
& +F_{16} \frac{\partial^{3} \phi_{\mathrm{x}}}{\partial x^{2} \partial y}+F_{26} \frac{\partial^{3} \phi_{\mathrm{y}}}{\partial x \partial y^{2}}+F_{66}\left(\frac{\partial^{3} \phi_{\mathrm{y}}}{\partial x^{2} \partial y}+\frac{\partial^{3} \phi_{\mathrm{x}}}{\partial x \partial y^{2}}\right)-c_{1} H_{16}\left(\frac{\partial^{3} \phi_{\mathrm{x}}}{\partial x^{2} \partial y}+\frac{\partial^{4} w_{0}}{\partial x^{3} \partial y}\right) \\
& -c_{1} H_{26}\left(\frac{\partial^{3} \phi_{\mathrm{y}}}{\partial x \partial y^{2}}+\frac{\partial^{4} w_{0}}{\partial x \partial y^{3}}\right)-c_{1} H_{66}\left(\frac{\partial^{3} \phi_{\mathrm{y}}}{\partial x^{2} \partial y}+\frac{\partial^{3} \phi_{\mathrm{x}}}{\partial x \partial y^{3}}+2 \frac{\partial^{4} w_{0}}{\partial x^{2} \partial y^{2}}\right) \\
& \frac{\partial M_{\mathrm{x}}}{\partial x}=B_{11}\left(\frac{\partial w_{0}}{\partial x} \frac{\partial^{2} w_{0}}{\partial x^{2}}+\frac{\partial^{2} u_{0}}{\partial x^{2}}\right)+B_{12}\left(\frac{\partial^{2} w_{0}}{\partial x \partial y} \frac{\partial w_{0}}{\partial y}+\frac{\partial^{2} v_{0}}{\partial x \partial y}\right)+B_{16}\left(\frac{\partial w_{0}}{\partial x} \frac{\partial^{2} w_{0}}{\partial x \partial y}+\frac{\partial^{2} w_{0}}{\partial x^{2}} \frac{\partial w_{0}}{\partial y}+\frac{\partial^{2} u_{0}}{\partial x \partial y}+\frac{\partial^{2} v_{0}}{\partial x^{2}}\right) \\
& +D_{11} \frac{\partial^{2} \phi_{\mathrm{x}}}{\partial x^{2}}+D_{12} \frac{\partial^{2} \phi_{\mathrm{y}}}{\partial x \partial y}+D_{16}\left(\frac{\partial^{2} \phi_{\mathrm{y}}}{\partial x^{2}}+\frac{\partial^{2} \phi_{\mathrm{x}}}{\partial x \partial y}\right)-c_{1} F_{11}\left(\frac{\partial^{2} \phi_{\mathrm{x}}}{\partial x^{2}}+\frac{\partial^{3} w_{0}}{\partial x^{3}}\right)-c_{1} F_{12}\left(\frac{\partial^{2} \phi_{\mathrm{y}}}{\partial x \partial y}+\frac{\partial^{3} w_{0}}{\partial x \partial y^{2}}\right) \\
& -c_{1} F_{16}\left(\frac{\partial^{2} \phi_{\mathrm{y}}}{\partial x^{2}}+\frac{\partial^{2} \phi_{\mathrm{x}}}{\partial x \partial y}+2 \frac{\partial^{3} w_{0}}{\partial x^{2} \partial y}\right)-\frac{\partial M_{\mathrm{x}}^{T}}{\partial x} \\
& \frac{\partial M_{\mathrm{xy}}}{\partial y}=B_{16}\left(\frac{\partial w_{0}}{\partial x} \frac{\partial^{2} w_{0}}{\partial x \partial y}+\frac{\partial^{2} u_{0}}{\partial x \partial y}\right)+B_{26}\left(\frac{\partial w_{0}}{\partial y} \frac{\partial^{2} w_{0}}{\partial y^{2}}+\frac{\partial^{2} v_{0}}{\partial y^{2}}\right)+B_{66}\left(\frac{\partial w_{0}}{\partial x} \frac{\partial^{2} w_{0}}{\partial y^{2}}+\frac{\partial^{2} w_{0}}{\partial x \partial y} \frac{\partial w_{0}}{\partial y}+\frac{\partial^{2} u_{0}}{\partial y^{2}}+\frac{\partial^{2} v_{0}}{\partial x \partial y}\right) \\
& +D_{16} \frac{\partial^{2} \phi_{\mathrm{x}}}{\partial x \partial y}+D_{26} \frac{\partial^{2} \phi_{\mathrm{y}}}{\partial y^{2}}+D_{66}\left(\frac{\partial^{2} \phi_{\mathrm{y}}}{\partial x \partial y}+\frac{\partial^{2} \phi_{\mathrm{x}}}{\partial y^{2}}\right)-c_{1} F_{16}\left(\frac{\partial^{2} \phi_{\mathrm{x}}}{\partial x \partial y}+\frac{\partial^{3} w_{0}}{\partial x^{2} \partial y}\right)-c_{1} F_{26}\left(\frac{\partial^{2} \phi_{\mathrm{y}}}{\partial y^{2}}+\frac{\partial^{3} w_{0}}{\partial y^{3}}\right) \\
& -c_{1} F_{66}\left(\frac{\partial^{2} \phi_{\mathrm{y}}}{\partial x \partial y}+\frac{\partial^{2} \phi_{\mathrm{x}}}{\partial y^{2}}+2 \frac{\partial^{3} w_{0}}{\partial x \partial y^{2}}\right)
\end{aligned}
$$




$$
\begin{aligned}
\frac{\partial M_{y}}{\partial y}= & B_{12}\left(\frac{\partial w_{0}}{\partial x} \frac{\partial^{2} w_{0}}{\partial x \partial y}+\frac{\partial^{2} u_{0}}{\partial x \partial y}\right)+B_{22}\left(\frac{\partial^{2} w_{0}}{\partial y^{2}} \frac{\partial w_{0}}{\partial y}+\frac{\partial^{2} v_{0}}{\partial y^{2}}\right)+B_{26}\left(\frac{\partial w_{0}}{\partial x} \frac{\partial^{2} w_{0}}{\partial y^{2}}+\frac{\partial^{2} w_{0}}{\partial x \partial y} \frac{\partial w_{0}}{\partial y}+\frac{\partial^{2} u_{0}}{\partial y^{2}}+\frac{\partial^{2} v_{0}}{\partial x \partial y}\right) \\
+ & D_{12} \frac{\partial^{2} \phi_{\mathrm{x}}}{\partial x \partial y}+D_{22} \frac{\partial^{2} \phi_{\mathrm{y}}}{\partial y^{2}}+D_{26}\left(\frac{\partial^{2} \phi_{\mathrm{y}}}{\partial x \partial y}+\frac{\partial^{2} \phi_{\mathrm{x}}}{\partial y^{2}}\right)-c_{1} F_{12}\left(\frac{\partial^{2} \phi_{\mathrm{x}}}{\partial x \partial y}+\frac{\partial^{3} w_{0}}{\partial x^{2} \partial y}\right)-c_{1} F_{22}\left(\frac{\partial^{2} \phi_{\mathrm{y}}}{\partial y^{2}}+\frac{\partial^{3} w_{0}}{\partial y^{3}}\right) \\
& -c_{1} F_{26}\left(\frac{\partial^{2} \phi_{\mathrm{y}}}{\partial x \partial y}+\frac{\partial^{2} \phi_{\mathrm{x}}}{\partial y^{2}}+2 \frac{\partial^{3} w_{0}}{\partial x \partial y^{2}}\right)-\frac{\partial M_{\mathrm{y}}^{T}}{\partial y} \\
\frac{\partial M_{\mathrm{xy}}}{\partial x}= & B_{16}\left(\frac{\partial w_{0}}{\partial x} \frac{\partial^{2} w_{0}}{\partial x^{2}}+\frac{\partial^{2} u_{0}}{\partial x^{2}}\right)+B_{26}\left(\frac{\partial w_{0}}{\partial y} \frac{\partial^{2} w_{0}}{\partial x \partial y}+\frac{\partial^{2} v_{0}}{\partial x \partial y}\right)+B_{66}\left(\frac{\partial w_{0}}{\partial x} \frac{\partial^{2} w_{0}}{\partial x \partial y}+\frac{\partial^{2} w_{0}}{\partial x^{2}} \frac{\partial w_{0}}{\partial y}+\frac{\partial^{2} u_{0}}{\partial x \partial y}+\frac{\partial^{2} v_{0}}{\partial x^{2}}\right) \\
+ & D_{16} \frac{\partial^{2} \phi_{\mathrm{x}}}{\partial x^{2}}+D_{26} \frac{\partial^{2} \phi_{\mathrm{y}}}{\partial x \partial y}+D_{66}\left(\frac{\partial^{2} \phi_{\mathrm{y}}}{\partial x^{2}}+\frac{\partial^{2} \phi_{\mathrm{x}}}{\partial x \partial y}\right)-c_{1} F_{16}\left(\frac{\partial^{2} \phi_{\mathrm{x}}}{\partial x^{2}}+\frac{\partial^{3} w_{0}}{\partial x^{3}}\right)-c_{1} F_{26}\left(\frac{\partial^{2} \phi_{\mathrm{y}}}{\partial x \partial y}+\frac{\partial^{3} w_{0}}{\partial x \partial y^{2}}\right) \\
-c_{1} F_{66}\left(\frac{\partial^{2} \phi_{\mathrm{y}}}{\partial x^{2}}+\frac{\partial^{2} \phi_{\mathrm{x}}}{\partial x \partial y}+2 \frac{\partial^{3} w_{0}}{\partial x^{2} \partial y}\right) & (\mathrm{A} .26)
\end{aligned}
$$

